SERVIÇO DE PÓS-GRADUAÇÃO DO ICMC-USP

Data de Depósito:

Assinatura:

\title{
Desenvolvimento de um Método Numérico para Simular Escoamentos Viscoelásticos \\ Axissimétricos com superfícies livres
}

\author{
Marcela Alexandra da Silva
}

Orientador: Prof. Dr. Murilo Francisco Tomé

Dissertação apresentada ao Instituto de Ciências Matemáticas e de Computação - ICMC-USP, como parte dos requisitos para obtenção do título de Mestre em Ciências de Computação e Matemática Computacional.

USP - São Carlos

Abril $/ 2005$ 
Aluno: Marcela Alexandra da Silva

A Comissão Julgadora:

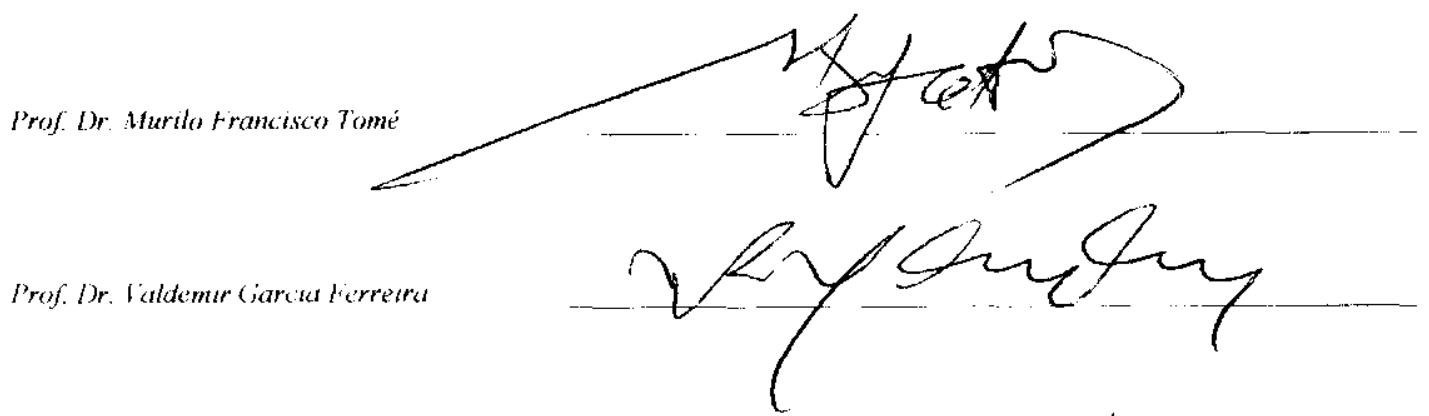

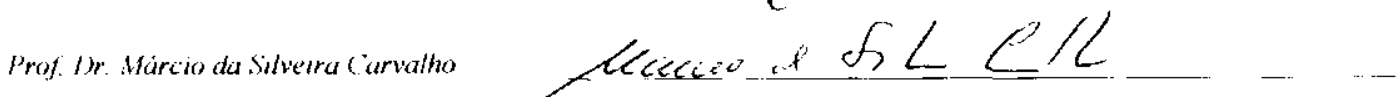


Aos meus pais Wilson e Alzira, a minha irmã Dani e a.o meu noivo Wesley. 


\section{Agradecimentos}

Agradeço a todas pessoas que me ajudaram a vencer mais esta etapa da vida e que de alguma maneira contribuíram para a realização deste trabalho. Peço desculpas antecipadamente àqueles que por algum motivo esqueci de citar.

Aos meus pais, Wilson e Alzira e minha irmã Daniela, por sempre acreditarem em mim e no meu potencial. Por estarem sempre presentes, mesmo sendo a distância muito grande, e junto comigo enfrentaram todas as dificuldades e alegrias nestes dois anos e de toda a minha vida. Pelo carinho, amor, orações, dedicação e atenção. Amo muito vocês!

Ao meu noivo Wesley, pelo amor, carinho, paciência, por estar sempre ao meu lado e pelas palavras de conforto nos momentos difíceis.

Ao meu orientador Prof. Murilo Francisco Tomé pelo conhecimento compartilhado, pela orientação e incentivo no decorrer deste trabalho. Aos professores do grupo de pesquisa pelo conhecimento compartilhado.

Aos amigos e professores do curso de Matemática da UNESP de Presidente Prudente que sempre estiveram presentes, em especial a Mariana, Rodrigo Miyasaki, Nair, Fernando e ao Prof. José Roberto Nogucira pelo inıcentivo c orientação durante os dois anos de Iniciação Científica.

As grandes e sempre amigas Gil e Day pela amizade e apoio. Vocês são muito especiais!

Aos amigos de São Carlos e do LCAD, em especial a Analice e Fernanda (pela amizade e companheirismo durante as disciplinas), Cássio, João Paulo, Fernando, Pepê, Kemelli, Helton, Marcello, Igor, Alex, Silvino, pela amizade e por terem feito esses anos mais alegres e descontraídos.

A FAPESP, pelo apoio financeiro no desenvolvimento deste trabalho.

A Dcus, sobretudo, por estar sempre comigo me iluminando durante todas as dificuldades e alegrias nıa realização deste trabalho e de toda a minha vida. 


\section{Resumo}

Yeste trabalho são apresentadas as equações governantes para um fluido Oldroyd-B juntamente com as condições de contorno para escoamentos viscoelásticos axissimétricos com superfícies livres. Um método numérico para simular escoamentos com superfícies livres é apresentado e as equações resultantes são resolvidas utilizando o método de diferenças finitas numa malha deslocada. São desenvolvidas formulações para o cálculo do tensor cxtra-tcnsão em contornos rígidos e no eixo de simetria. As condições de contorno na superfície livre são discutidas em detalhes. Os termos convectivos são aproximados pelo método 'upwind' de alta ordem CUBISTA ('A convergent and universally bounded interpolation scheme for the treatment of advection'). O fluido é modelado utilizando a técnica 'Marker-and-Cell' o que permite visualizar e localizar a superfície livre do fluido. Para evitar ondulações, a superfície livre é suavizada pela técnica TSUR ('Trapezoidal Surface Removal'). O método numérico descrito neste trabalho foi implementado no sistema de simulação Freeflow-AXI c validado comparando os resultados numéricos do escoamento em um tubo com a respectiva solução analítica. Resultados numéricos incluem: simulação do inchamento do extrudado, gota incidindo contra uma superfície rígida e a simulação do 'splashing drop' para vários números de Reynolds e de Weissenberg. 


\section{Abstract}

This work presents the governing equations together with the corresponding boundary conditions for the flow of an Oldroyd-B fluid with free surfaces in axisymmetric geometries. A numerical method for simulating free surface flows is presented and the resulting equations are solved by the finite difference method on a staggered grid. A formulation for the computation of the extra-stress tensor on rigid boundaries and on the symmetry axis is devcloped. The boundary conditions of the free surface are discussed in details. One feature of the numerical technique presented in this work is the approximation of the convective terms appearing in the equations of motion and in the constitutive equation by the high order CUBISTA scheme (A convergent and universally bounded interpolation scheme for the treatment of advection). The fluid is modeled by the Marker-and-Cell method which permits the visualization and the location of the free surface. In order to avoid ondulations the frec surface is smoothed by the TSLR (Trapezoidal Surface Removal) method which is a mass conserving procedure. The resulting difference equations are then implemented into the Freeflow-AXI simulation system. The code implementation is validated by simulating the flow of an Oldroyd-B fluid in a pipe. Numerical results include the simulation of the transient extrudate swell, impacting drop and the splashing drop of an Oldroyd-B fluid at high Reynolds numbers and various Weissenberg numbers. 


\section{Sumário}

$\begin{array}{ll}\text { Introdução } & 1\end{array}$

1 Equações Governantes 3

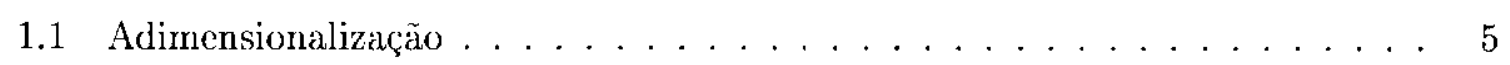

1.2 Condições de Contorno . . . . . . . . . . . . . . . 6

1.2.1 Cálculo do Tensor extra-tensão em Contornos Rígidos . . . . . . . 7

1.2.2 Cálculo do Tensor extra-tensão no Eixo de Simetria . . . . . . . . . . 10

1.2.3 Cálculo do Tensor extra-tensão em 'Inflows' e 'Outflows' . . . . . . . 11

1.2.4 Condições de Contorno na Superfície Livre . . . . . . . . . . . . 12

2 Método Numérico 13

2.1 Algoritmo Computacional . . . . . . . . . . . . . . . 13

3 Aproximação por Diferenças Finitas $\quad 16$

3.1 Classificação das Células . . . . . . . . . . . . . . . . . . . . . 16

3.2 Discretização das Equações . . . . . . . . . . . . . . . . . . . 17

3.3 Aproximação da Equação de Poisson para $\psi$. . . . . . . . . . . 21

3.4 Aproximação da Equação da Velocidade Final . . . . . . . . . . . . . . 21

3.5 Aproximação da Equação da Pressão . . . . . . . . . . . . . . . . . . . . . . . . . . . 22

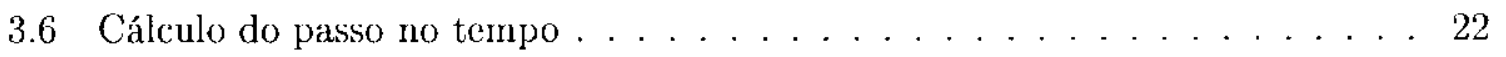

3.7 Discretização dos Termos Convectivos . . . . . . . . . . . . . . . 22

3.8 Aproximação das Condições de Contorno na Superfície Livre . . . . . . . . 25

3.9 Cálculo das Componentes do Tensor extra-tensão em Contornos Rígidos . . . 28

3.10 Cálculo das Componentes do Tensor extra-tensão no Fixo de Simetria . . . . 33

3.11 Cálculo das Componentes do Tensor extra-tensão em 'Inflows' e 'Outflows' . 35

4 Resultados Numéricos e Validação do Método Numérico 37

4.1 Validação do Método Numérico . . . . . . . . . . . . . . . . . . 37

4.2 Resultados Numéricos . . . . . . . . . . . . . . . . . . . . . 41

4.2.1 Simulação Numérica do Inchamento do Extrudado . . . . . . . . . . 41

4.3 Simulação Numérica do Impacto de uma Gota contra uma Superfície Rígida 64 
4.4 Simulação Numérica do 'splashing drop'

Conclusão

Referências Bibliográficas 


\section{Introdução}

A modelagem de escoamentos com superfícies livres tem sido urna área de intensa pesquisa nos últimos tempos e ainda apresenta vários desafios: o escoamento é transiente, não-newtoniano e envolve múltiplas superfícies em movimento. Apesar disso, muitos pesquisadores têm desenvolvido algoritmos numéricos que têm ajudado no projeto e manufatura de 'injection-moulded products'. Dentre as técricas numéricas empregadas, o método de diferenças finitas tem sido utilizado com sucesso por vários pesquisadores

Recentemente, o grupo de pesquisa em Matemática Computacional do ICMC-USP de São Carlos desenvolveu um ambiente de simulação de escoamentos incompressíveis tridimensionais denominado Freeflow-3D [9]. Frecflow-3D é um software construído especificamente para simular escoamentos newtonianos incompressiveis com superfícies livres em 3 dimensões. Esse ambiente é constituído de 3 módulos: Modflow-3D, Simflow-3D e Visflow-3D. O módulo Modflow-3D é responsável pela modelagem de objetos tais como 'containers', injetores e especificação do problema (por ex. especificação do domínio, atribuição das constantes adimensionais, inicialização de variávcis, etc.). Simflow-3D é a parte central do Freeflow-3D, pois esse módulo implementa a solução computacional das equações governantes para um fluido newtoniano incompressível utilizando o mćtodo de diferenças finitas. O módulo Visflow-3D disponibiliza a visualização dos dados gerados pelo módulo Simflow3D utilizando ferramentas de computação gráfica. Detalhıs sobre esses 3 módulos podem ser encontrados cm [9]. No entanto, na prática muitos problemas podem ser modelados como escoamentos com simetria radial e, portanto, uma versão denominada Freeflow-AXI [10] foi desenvolvida. Frecllow-AXI resolve as equações governantes de escoamentos newtonianos com superfícies livres numa malha diferenciada utilizando o método de diferenças finitas. Como em Freeflow-3D, o fluido é modelado pela técnica 'Marker-and-Cell' utilizando técnicas de modelagem geométrica [29]. Como em Frecflow-3D, Freeflow-AXI possui 3 módulos distintos: Modflow-AXI, Simflow-AXI e Visflow-AXI. Uma descrição detalhada de FreeflowAXI pode ser encontrada em [10]. Mais recentemente, Tomé et al. [12] desenvolveram um método numérico para resolver as equações governantes para escoamentos bidimensionais viscoelásticos. Mais especificamente, Tomé et al. [12] apresentaram um método numérico utilizando diferenças finitas para simular escoamentos bidimensionais com superfícies livres de um fluido Oldroyd-B. 
Este trabalho tem por finalidade estender a metodologia apresentada por Tomé $e$ a al. [12| para escoamentos viscoelásticos com simetria radial e sua implementação no sistema Freeflow-AXI. Pretende-se também simular problemas de interesse tais como: inchamento do extrudado, 'splashing drop' entre outros.

Este trabalho está organizado em 4 capítulos:

- Capítulo 1: são apresentadas as equações governantes para um fluido Oldroyd-B juntamente com as condições de contorno e condições na superfície livre;

- Capítulo 2: é apresentado um método numérico para resolver escoamentos viscoelásticos axissimétricos com superfícies livres;

- Capítulo 3: são apresentadas as aproximações por diferenças finitas das equações governantes e das condiçōes de contorno;

- Capítulo 4: são apresentados resultados numéricos bem como uma validação do método numérico desenvolvido nesse trabalho.

Por último, são apresentadas as considerações finais. 


\section{Capítulo 1}

\section{Equações Governantes}

As equações governantes para escoamentos viscoelásticos usando o modelo Oldroyd-B são as equações de conservação de quantidade de movimento

$$
\rho_{0}\left[\frac{\partial \mathbf{u}}{\partial t}+\nabla \cdot\left(\mathbf{u u}^{T}\right)\right]=\nabla \cdot \boldsymbol{\sigma}+\rho_{0} \mathbf{g}
$$

juntamente com as equações constitutivas

$$
\begin{gathered}
\boldsymbol{\sigma}=-p \mathbf{I}+\boldsymbol{\tau}, \\
\boldsymbol{\tau}+\lambda_{1} \stackrel{\nabla}{\tau}=2 \mu_{0}\left(\mathbf{D}+\lambda_{2} \stackrel{\vee}{\mathbf{D}}\right)
\end{gathered}
$$

e a equação da continuidade (assumindo incompressibilidade do fluido)

$$
\nabla \cdot \mathbf{u}=0
$$

onde $\sigma$ é o tensor de tensões totais, $p$ é a pressão, I é o tensor unitário, $\tau$ é o tensor extra-tensão simétrico e

$$
\mathbf{D}=\frac{1}{2}\left[(\nabla \mathbf{u})+(\nabla \mathbf{u})^{T}\right]
$$

é o tensor taxa de deformação. Na equação (1.3), $\mu_{0}$ é a viscosidade newtoniana ou viscosidade a zero cisalhamento e na equação (1.1) $\mu_{0}$ é a massa específica; $\lambda_{1}$ e $\lambda_{2}$ são constantes temporais de relaxação e retardo do movimento, respectivamente. Na equação (1.3), $\stackrel{\nabla}{\tau}$ representa a derivada contra-variante de $\tau$ (Crochet \& Walters [13]) e é definida por:

$$
\stackrel{\nabla}{\tau}=\frac{\partial \mathbf{u}}{\partial t}+\mathbf{u} \cdot(\nabla \boldsymbol{\tau})-(\nabla \mathbf{u})^{T} \cdot \boldsymbol{\tau}-\boldsymbol{\tau} \cdot(\nabla \mathbf{u})
$$

Para resolver as equações (1.1) - (1.4), introduzimos a mudança de variáveis (conhecida como transformação EVSS - 'Elastic-viscous stress-splitting' [8])

$$
\boldsymbol{\tau}=2 \mu_{0}\left(\frac{\lambda_{2}}{\lambda_{1}}\right) \mathbf{D}+\mathbf{S}
$$


onde $\mathbf{S}$ represent.a a contribuição não-newtoniana ao tensor extra-tensão. Substituindo (1.6) e (1.7) em (1.1) e (1.3), obtemos as equações

$$
\begin{gathered}
\mathbf{S}+\lambda_{1}\left(\frac{\partial \mathbf{S}}{\partial t}+\mathbf{u} \cdot \nabla \mathbf{S}-(\nabla \mathbf{u})^{T} \cdot \mathbf{S}-\mathbf{S} \cdot(\nabla \mathbf{u})\right)=2 \mu_{0}\left(1-\frac{\lambda_{2}}{\lambda_{1}}\right) \mathbf{D} \\
\rho_{0} \frac{D \mathbf{u}}{D t}=-\nabla p+2 \mu_{0}\left(\frac{\lambda_{2}}{\lambda_{1}}\right) \nabla \cdot \mathbf{D}+\nabla \cdot \mathbf{S}+\rho_{0} \mathbf{g}
\end{gathered}
$$

Neste trabalho vamos considerar escoamentos axissimétricos $(\partial / \partial \theta)=0$ usando coordenadas cilíndricas Orz, com $\mathbf{u}=u(r, z, t) \boldsymbol{e}_{\boldsymbol{r}}+v(r, z, t) \boldsymbol{e}_{\boldsymbol{z}}$. Deste modo, as equações de conservação de massa, conservação de quantidade de movimento e das componentes do tensor extra-tensão podem ser escritas como

$$
\begin{aligned}
& \frac{1}{r} \frac{\partial}{\partial r}(r u)+\frac{\partial v}{\partial z}=0 \\
& \frac{\partial u}{\partial t}+\frac{1}{r} \frac{\partial\left(r u^{2}\right)}{\partial r}+\frac{\partial(u v)}{\partial z}=-\frac{1}{\rho_{0}} \frac{\partial p}{\partial r}+\nu_{0}\left(\frac{\lambda_{2}}{\lambda_{1}}\right)\left[\frac{1}{r} \frac{\partial}{\partial r}\left(r \frac{\partial u}{\partial r}\right)+\frac{\partial^{2} u}{\partial z^{2}}-\frac{u}{r^{2}}\right] \\
& +\frac{1}{\rho_{0}}\left(\frac{1}{r} \frac{\partial}{\partial r}\left(r S^{r r}\right)+\frac{\partial S^{z r}}{\partial z}-\frac{S^{\theta \theta}}{r}\right)+g_{r}, \\
& \frac{\partial v}{\partial t}+\frac{1}{r} \frac{\partial(r u v)}{\partial r}+\frac{\partial\left(v^{2}\right)}{\partial z}=-\frac{1}{\rho_{0}} \frac{\partial p}{\partial z}+\nu_{0}\left(\frac{\lambda_{2}}{\lambda_{1}}\right)\left[\frac{1}{r} \frac{\partial}{\partial r}\left(r \frac{\partial v}{\partial r}\right)+\frac{\partial^{2} v}{\partial z^{2}}\right] \\
& +\frac{1}{\rho_{0}}\left(\frac{1}{r} \frac{\partial}{\partial r}\left(r S^{r z}\right)+\frac{\partial S^{z z}}{\partial z}\right)+g_{z} \\
& S^{r r}+\lambda_{1}\left(\frac{\partial S^{r r}}{\partial t}+\frac{1}{r} \frac{\partial\left(r u S^{r r}\right)}{\partial r}+\frac{\partial\left(v S^{r r}\right)}{\partial z}-2 \frac{\partial u}{\partial r} S^{r r}-2 \frac{\partial u}{\partial z} S^{r z}\right)=2 \mu_{0}\left(1-\frac{\lambda_{2}}{\lambda_{1}}\right) \frac{\partial u}{\partial r}, \\
& S^{z z}+\lambda_{1}\left(\frac{\partial S^{z z}}{\partial \ell}+\frac{1}{r} \frac{\partial\left(r u S^{z z}\right)}{\partial r}+\frac{\partial\left(v S^{z z}\right)}{\partial z}-2 \frac{\partial v}{\partial r} S^{r z}-2 \frac{\partial v}{\partial z} S^{z z}\right)=2 \mu_{0}\left(1-\frac{\lambda_{2}}{\lambda_{1}}\right) \frac{\partial v}{\partial z} \\
& S^{\theta \theta}+\lambda_{1}\left(\frac{\partial S^{\theta \theta}}{\partial t}+\frac{1}{r} \frac{\partial\left(r u S^{\theta \theta}\right)}{\partial r}+\frac{\partial\left(v S^{\theta \theta}\right)}{\partial z}-2 \frac{u}{r} S^{\theta \theta}\right)=2 \mu_{0}\left(1-\frac{\lambda_{2}}{\lambda_{1}}\right) \frac{u}{r} \\
& S^{r z}+\lambda_{1}\left(\frac{\partial S^{r z}}{\partial t}+\frac{1}{r} \frac{\partial\left(r u S^{r z}\right)}{\partial r}+\frac{\partial\left(v S^{r z}\right)}{\partial z}-\frac{\partial v}{\partial r} S^{r r}-\frac{\partial u}{\partial z} S^{z z}+\frac{u}{r} S^{r z}\right)= \\
& \mu_{0}\left(1-\frac{\lambda_{2}}{\lambda_{1}}\right)\left(\frac{\partial u}{\partial z}+\frac{\partial v}{\partial r}\right)
\end{aligned}
$$

Nas equações (1.11) - (1.12), temos que $\nu_{0}=\mu_{0} / \rho_{0}$ é o coeficiente de viscosidade cinemática e $g_{r}, g_{z}$ são as componentes gravitacionais nas direçôes $r$ e $z$, respectivamente. 


\subsection{Adimensionalização}

Em CFD ć descjávcl cmpregar formas adimensionais das equações de conservação com o objetivo de mostrar os efeitos físicos contidos nas equações, formular o modelo independente do sistema de unidades, limitar os valores de variáveis e parâmetros e principalmente fornecer condições para se obter situações geometricamente similares. Para isso, considerase uma mudança de variáveis, surgindo então alguns grupos adimensionais como o número de Reynolds, de Froude e o de Weissenberg. Assim,

- Número de Reynolds (Re): Representa a razão entre as forças inerciais (que são responsáveis pelo movimento do fluido) c as forças viscosas (que são responsáveis pela dissipação devido ao coeficiente de viscosidade molecular) do escoamento, dada por

$$
R e=\frac{\rho_{0} L U}{\mu_{0}}=\frac{L U}{\nu_{0}},
$$

onde $L$ e $U$ são as unidades escalares de comprimcnto e velocidade, respectivamente.

- Número de Froude $\left(F_{r}\right)$ : Representa a razão entre as forças inerciais e as forças gravitacionais, ou seja,

$$
F_{r}=\frac{U}{\sqrt{g L}}
$$

- Número de Weissenberg (We): Relaciona dois tempos característicos: o tempo de relaxação $\lambda_{1}$ e o tempo $\frac{L}{U}$ que é característico para o problema e é dado por

$$
W e=\frac{\lambda_{1} U}{L} .
$$

Para adimensionalizar as equações de quantidade de movimento, continuidade e a equação constitutiva definimos as variáveis adimensionais, como segue.

$$
\begin{gathered}
u=U \bar{u}, \quad v=U \bar{v}, \quad r=L r, \\
z=L \bar{z}, \quad t=\frac{L}{U} \bar{l}, \quad \nu=\nu_{0} \bar{\nu}, \\
p=\rho_{0} U^{2} \bar{p}, \quad \mathbf{S}=\rho_{0} U^{2} \overline{\mathbf{S}}, \quad \mathbf{g}=g \mathbf{g} .
\end{gathered}
$$

Substituindo as variáveis adimensionais nas equações (1.10) - (1.16) obtemos as equações na forma adimensional (as barras foram omitidas por simplicidade).

$$
\frac{1}{r} \frac{\partial}{\partial r}(r u)+\frac{\partial v}{\partial z}=0
$$




$$
\begin{aligned}
& \frac{\partial u}{\partial t}+\frac{1}{r} \frac{\partial\left(r u^{2}\right)}{\partial r}+\frac{\partial(u v)}{\partial z}=-\frac{\partial p}{\partial r}+\frac{1}{R e}\left(\frac{\lambda_{2}}{\lambda_{1}}\right)\left[\frac{1}{r} \frac{\partial}{\partial r}\left(r \frac{\partial u}{\partial r}\right)+\frac{\partial^{2} u}{\partial z^{2}}-\frac{u}{r^{2}}\right] \\
& +\left(\frac{1}{r} \frac{\partial}{\partial r}\left(r S^{r r}\right)+\frac{\partial S^{z r}}{\partial z}-\frac{S^{\theta \theta}}{r}\right)+\frac{1}{F_{r}^{2}} g_{r} \\
& \frac{\partial v}{\partial t}-\frac{1}{r} \frac{\partial(r u v)}{\partial r}+\frac{\partial\left(v^{2}\right)}{\partial z}=-\frac{\partial p}{\partial z}+\frac{1}{R c}\left(\frac{\lambda_{2}}{\lambda_{1}}\right)\left[\frac{1}{r} \frac{\partial}{\partial r}\left(r \frac{\partial v}{\partial r}\right)-\frac{\partial^{2} v}{\partial z^{2}}\right] \\
& +\left(\frac{1}{r} \frac{\partial}{\partial r}\left(r S^{r z}\right)+\frac{\partial S^{z z}}{\partial z}\right)+\frac{1}{F_{r}^{2}} g_{z}, \\
& S^{r r}+W e\left(\frac{\partial S^{r r}}{\partial t}+\frac{1}{r} \frac{\partial\left(r u S^{r r}\right)}{\partial r}+\frac{\partial\left(v S^{r r}\right)}{\partial z}-2 \frac{\partial u}{\partial r} S^{r r}-2 \frac{\partial u}{\partial z} S^{r z}\right)=\frac{2}{R e}\left(1-\frac{\lambda_{2}}{\lambda_{1}}\right) \frac{\partial u}{\partial r}, \\
& S^{z z}+W e\left(\frac{\partial S^{z z}}{\partial t}+\frac{1}{r} \frac{\partial\left(r u S^{z z}\right)}{\partial r}+\frac{\partial\left(v S^{z z}\right)}{\partial z}-2 \frac{\partial v}{\partial r} S^{r z}-2 \frac{\partial v}{\partial z} S^{z z}\right)=\frac{2}{R e}\left(1-\frac{\lambda_{2}}{\lambda_{1}}\right) \frac{\partial v}{\partial z}, \\
& S^{\theta \theta}+W e\left(\frac{\partial S^{\theta \theta}}{\partial t}+\frac{1}{r} \frac{\partial\left(r u S^{+\theta \theta}\right)}{\partial r}+\frac{\partial\left(v S^{\theta \theta}\right)}{\partial z}-2 \frac{u}{r} S^{\theta \theta \theta}\right)=\frac{2}{R e}\left(1-\frac{\lambda_{2}}{\lambda_{1}}\right) \frac{u}{r}, \\
& S^{r z}+W e\left(\frac{\partial S^{r z}}{\partial t}+\frac{1}{r} \frac{\partial\left(r u S^{r z}\right)}{\partial r}+\frac{\partial\left(v S^{r z}\right)}{\partial z}-\frac{\partial v}{\partial r} S^{r r}-\frac{\partial u}{\partial z} S^{z z}+\frac{u}{r} S^{r z}\right)= \\
& \frac{1}{R e}\left(1-\frac{\lambda_{2}}{\lambda_{1}}\right)\left(\frac{\partial u}{\partial z}+\frac{\partial v}{\partial r}\right) \text {, }
\end{aligned}
$$

onde Re é o número de Rcynolds, $F_{r}$ ć o número de Froude e $W e$ é o número de Weisscriberg.

\subsection{Condições de Contorno}

Condições iniciais e de contorno fisicamente adequadas são impostas para resolver as equações (1.21) - (1.26).

Como condições iniciais do escoamento especificamos a distribuição espacial das variáveis dependentes no tempo $t=0$. As condiçoos iniciais do escoamento para as componentes de u c S serão adotadas como sendo nulas, isto é,

$$
\mathbf{u}=\mathbf{0} \quad \text { e } \quad \mathbf{S}=\mathbf{0}
$$


As condições de contorno em 'inflows' (entrada de fluido) e 'outflows' (saída de fluido) para a velocidade são dadas por

$$
\begin{gathered}
\text { 'Inflows': } u_{n}=U \text { e } u_{\tau}=0, \\
\text { 'Outflows': } \frac{\partial u_{n}}{\partial n}=0 \quad \text { e } \quad \frac{\partial u_{\tau}}{\partial n}=0,
\end{gathered}
$$

onde $n$ e $\tau$ denotam direções normais e tangenciais ao 'inflow' e 'outflow', respectivamente.

\subsubsection{Cálculo do Tensor extra-tensão em Contornos Rígidos}

Fm contornos rígidos, adotaremos a condição 'no-slip' (não-escorregamento)

$$
\mathbf{u}=\mathbf{u}_{c}
$$

onde $\mathbf{u}_{c}$ é a vclocidade do contorno rígido. Geralmente o contorno rígido está estacionário, então tomamos $\mathbf{u}_{c}=0$.

Os valores das componentes $S^{r r}, S^{z z}, S^{(t)}$ e $S^{r z}$ no contorno rígido serão obtidos por meio das equaçôes (1.23) - (1.26), para as quais primeiramente introduzimos a mudança de variável $\mathbf{S}=e^{\frac{-1}{W e} t \dot{\mathbf{S}}}$ (introduzida por 'lomé et al. [12]) obtendo as equações

$$
\begin{aligned}
& \left(\frac{\partial \hat{S}^{r r}}{\partial t}+\frac{1}{r} \frac{\partial\left(r u \tilde{S}^{r r}\right)}{\partial r}+\frac{\partial\left(v \tilde{S}^{r r}\right)}{\partial z}-2 \frac{\partial u}{\partial r} \tilde{S}^{r r}-2 \frac{\partial u}{\partial z} \tilde{S}^{r z}\right)=\frac{1}{R e} \frac{2}{W e}\left(1-\frac{\lambda_{2}}{\lambda_{1}}\right) e^{\frac{1}{W e}} t \frac{\partial u}{\partial r} \\
& \left(\frac{\partial \tilde{S}^{z z}}{\partial t}+\frac{1}{r} \frac{\partial\left(r u \tilde{S}^{z z}\right)}{\partial r}+\frac{\partial\left(v \tilde{S}^{z z}\right)}{\partial z}-2 \frac{\partial v}{\partial r} \tilde{S}^{r z}-2 \frac{\partial v}{\partial z} \tilde{S}^{z z}\right)=\frac{1}{R e} \frac{2}{W e}\left(1-\frac{\lambda_{2}}{\lambda_{1}}\right) e^{\frac{1}{W_{e}} t} \frac{\partial v}{\partial z} \\
& \left(\frac{\partial \tilde{S}^{\theta \theta}}{\partial l}+\frac{1}{r} \frac{\partial\left(r u \tilde{S}^{\theta \theta}\right)}{\partial r}+\frac{\partial\left(v \tilde{S}^{\theta \theta}\right)}{\partial z}-2 \frac{u}{r} \tilde{S}^{\theta \theta}\right)=\frac{1}{R e} \frac{2}{W e}\left(1-\frac{\lambda_{2}}{\lambda_{1}}\right) e^{\frac{1}{W e} t} \frac{u}{r} \\
& \left(\frac{\partial \tilde{S}^{r z}}{\partial t}+\frac{1}{r} \frac{\partial\left(r u \tilde{S}^{r z}\right)}{\partial r}+\frac{\partial\left(v \tilde{S}^{r z}\right)}{\partial z}-\frac{\partial v}{\partial r} \tilde{S}^{r r}-\frac{\partial u}{\partial z} \tilde{S}^{z z}+\frac{u}{r} \tilde{S}^{r z}\right)= \\
& \frac{1}{R e} \frac{1}{W e}\left(1-\frac{\lambda_{2}}{\lambda_{1}}\right) e^{\frac{1}{W r} t}\left(\frac{\partial u}{\partial z}+\frac{\partial v}{\partial r}\right) \text {. }
\end{aligned}
$$

Para resolvermos as equaçōes (1.30) - (1.33), vamos considerar contornos rígidos estacionários horizontais e verticais, como segue. 


\section{Contornos rígidos paralelos ao eixo $r$}

Nestes contornos aplicando as condições de não-escorregamento para as componentes da velocidade, temos que $u=v=0 \Longrightarrow \frac{\partial u}{\partial r}=\frac{\partial v}{\partial r}=0$ e pela equação da continuidade $\frac{\partial v}{\partial z}=0$

Assim, as equações de tensão (1.30) - (1.33) se reduzem a

$$
\begin{aligned}
\frac{\partial \tilde{S}^{r r}}{\partial t}-2 \frac{\partial u}{\partial z} \tilde{S}^{r z} & =0 \\
\frac{\partial \tilde{S}^{z z}}{\partial t} & =0 \\
\frac{\partial \tilde{S}^{\theta \theta}}{\partial t} & =0 \\
\frac{\partial \tilde{S}^{r z}}{\partial t}-\frac{\partial u}{\partial z} \tilde{S}^{z z} & =\frac{1}{R e} \frac{1}{W e}\left(1-\frac{\lambda_{2}}{\lambda_{1}}\right) e^{\frac{1}{W_{c}} t} \frac{\partial u}{\partial z}
\end{aligned}
$$

Agora, as equações (1.34) - (1.37) podem ser resolvidas para $\tilde{S}^{r r}, \tilde{S}^{z z} ; \tilde{S}^{z \prime \prime}$ e $\tilde{S}^{r z}$. Das equações (1.35) e (1.36) com condição inicial $\mathbf{S}=0$, obtemos $\tilde{S}^{\theta \theta}=0$ e $\tilde{S}^{z z}=0$. Assim

$$
S^{r \theta}(l+\delta l)=0 \quad \text { e } \quad S^{z z}(l+\delta l)=0
$$

Então, a equação (1.37) se reduz a

$$
\frac{\partial \tilde{S}^{r z}}{\partial t}=\frac{1}{R e} \frac{1}{W e}\left(1-\frac{\lambda_{2}}{\lambda_{1}}\right) e^{\frac{1}{W_{c}^{\prime}} t} \frac{\partial u}{\partial z} .
$$

Integrando a equação acima em $[t, t+\delta l]$, vem

$$
\tilde{S}^{r z}(t+\delta t)-\tilde{S}^{r z}(l)=\frac{1}{\operatorname{Re}} \frac{1}{W e}\left(1-\frac{\lambda_{2}}{\lambda_{1}}\right) \int_{l}^{t+\delta t} \frac{\partial u}{\partial z} e^{\frac{1}{W t} s} d s .
$$

Aplicando o Teorema do Valor Médio para integrais, obtemos

$$
\tilde{S}^{r z}(t+\delta l)-\tilde{S}^{r z}(t)=\frac{1}{\operatorname{Re}}\left(1-\frac{\lambda_{2}}{\lambda_{1}}\right) \frac{\partial u}{\partial z}\left(t^{*}\right) \int_{t}^{t+\delta t} \frac{1}{W e} e^{\frac{1}{W_{e}} s} d s
$$

onde $\frac{\partial u}{\partial z}\left(t^{*}\right)$ c avaliada em $t^{*} \in(t, t+\delta t)$. Assim, podemos escrever

$$
\tilde{S}^{r z}(t+\delta t)=\tilde{S}^{r z}(t)+\frac{1}{R c}\left(1-\frac{\lambda_{2}}{\lambda_{1}}\right) \frac{\partial u}{\partial z}\left(t^{*}\right) e^{\frac{1}{w^{2}} t}\left[e^{\frac{1}{w^{c}} \delta t}-1\right] .
$$

Da mudança de variável $S^{r z}(t+\delta t)=c \frac{1}{w_{e}}(t+\delta t) \tilde{S}^{r z}(t+\delta l)$, temos que o valor de $S^{r z}(t+\delta t)$ no contorno rígido paralelo ao eixo $r$ é dado por

$$
S^{r z}(t+\delta t)=e^{\frac{-1}{w^{*}} \delta t} S^{r z}(t)+\frac{1}{\operatorname{Re}}\left(1-\frac{\lambda_{2}}{\lambda_{1}}\right) \frac{\partial u}{\partial z}\left(t^{*}\right)\left[1-e^{\frac{-1}{w^{\prime}} \delta t}\right] .
$$


Para calcularmos $S^{r r}$ integramos a equação (1.34) em $[t, t+\delta l]$, o que fornece

$$
\tilde{S}^{r r}(t+\delta t)=\tilde{S}^{r r}(l)+2 \int_{t}^{t+\delta t} \frac{\partial u}{\partial z} \tilde{S}^{r z}(s) d s
$$

Aproximando a integral pela regra dos trapézios, temos

$$
\tilde{S}^{r r}(t+\delta t)=\tilde{S}^{r r}(t)+\delta t\left[\frac{\partial u}{\partial z}(t) \tilde{S}^{r z}(t)+\frac{\partial u}{\partial z}(t+\delta t) \tilde{S}^{r z}(t+\delta t)\right]
$$

Assim, da mudança de variável $S^{r z}(t+\delta t)=e^{\frac{1}{w^{\prime}}(t+\delta t)} \tilde{S}^{r z}(t+\delta t)$, obtemos

$$
S^{r r}(t+\delta t)=e^{\frac{-1}{W^{\prime} t} \delta t} S^{r r}(t)+\delta l\left[\frac{\partial u}{\partial z}(l) e^{\frac{-1}{w_{e}} \partial t} S^{r z}(t)+\frac{\partial u}{\partial z}(t+\delta t) S^{r z}(t+\delta t)\right]
$$

\section{Contornos rígidos paralelos ao eixo $z$}

Do mesmo modo, aplicando as condições de não-escorregamento sobre o contorno vertical temos que $u=v=0 \Longrightarrow \frac{\partial u}{\partial z}=\frac{\partial v}{\partial z}=0$ e pela equação da continuidade $\frac{\partial u}{\partial r}=0$.

Substituindo esses valores nas equações (1.30) - (1.33), obtemos

$$
\begin{aligned}
\frac{\partial \tilde{S}^{r r}}{\partial t} & =0 \\
\frac{\partial \tilde{S}^{\theta \theta}}{\partial t} & =0 \\
\frac{\partial \tilde{S}^{z z}}{\partial t}-2 \frac{\partial v}{\partial r} \tilde{S}^{r z} & =0 \\
\frac{\partial \tilde{S}^{r z}}{\partial t}-\frac{\partial v}{\partial r} \tilde{S}^{r r} & =\frac{1}{R e} \frac{1}{W e}\left(1-\frac{\lambda_{2}}{\lambda_{1}}\right) e^{\frac{1}{W_{e}} t} \frac{\partial v}{\partial r}
\end{aligned}
$$

Da mesma mancira como foi feito para calcular os valores das componentes do tensor extra-tensão no contorno rígido paralelo ao eixo $r$, podemos mostrar que os valores de $S^{r r}$, $S^{z z}, S^{\theta \theta}$ e $S^{r z}$ no contorno rígido paralelo ao eixo $z$ são dados por

$$
\begin{aligned}
S^{r r}(l+\delta l) & =0 \\
S^{\theta \theta}(l+\delta t) & =0 \\
S^{r z}(t+\delta t) & =e^{\frac{-1}{W+} \delta t} S^{r z}(l)+\frac{1}{R e}\left(1-\frac{\lambda_{2}}{\lambda_{1}}\right) \frac{\partial v}{\partial r}\left(t^{*}\right)\left[1-e^{\frac{1}{W_{e}} \delta t}\right] \\
S^{z z}(t+\delta t) & =e^{\frac{1}{W e} \delta t} S^{z z}(t)+\delta t\left[\frac{\partial v}{\partial r}(t) e^{\frac{-1}{W_{t}} \delta t} S^{r z}(l)+\frac{\partial v}{\partial r}(l+\delta l) S^{r z}(l+\delta t)\right]
\end{aligned}
$$




\subsubsection{Cálculo do Tensor extra-tensão no Eixo de Simetria}

Neste trabalho, o domínio computacional de resolução de um problema fica delimitado pela superfície que representa o eixo de simetria e os outros contornos existentes, pois estamos trabalhando com escoamentos axissimétricos.

Será aplicada a condição de escorregamento para as componentes de velocidade sobre a superfície de simetria, (onde $r=0$ ). Esta condição garante que o fluido não transpõe a fronteira de simetria e não ocorre perda friccional na região adjaccnte à frontcira, ou scja,

$$
u(r, z, t)=0, \quad \frac{\partial v(r, z, t)}{\partial r}=0
$$

assumindo que o eixo de simetria é vertical.

Para as componentes do tensor extra-tensão $S^{r r}, S^{r z z}, S^{r \theta}$ e $S^{r z}$ aplicamos as condições (1.49) nas equações (1.30) - (1.33) as quais reduzem a

$$
\begin{aligned}
\frac{\partial \tilde{S}^{r r}}{\partial t} & =-\frac{\partial\left(v \tilde{S}^{r r}\right)}{\partial z}+2 \frac{\partial u}{\partial r} \tilde{S}^{r r}+\frac{1}{R e} \frac{2}{W e}\left(1-\frac{\lambda_{2}}{\lambda_{1}}\right) e^{\frac{1}{W^{r} c}} \frac{\partial u}{\partial r} \\
\frac{\partial \tilde{S}^{z z}}{\partial t} & =-\frac{\partial\left(v \tilde{S}^{z z}\right)}{\partial z}+2 \frac{\partial v}{\partial z} \tilde{S}^{z z}+\frac{1}{R e} \frac{2}{W e}\left(1-\frac{\lambda_{2}}{\lambda_{1}}\right) e^{\frac{1}{W e}} \frac{\partial v}{\partial z} \\
\frac{\partial \tilde{S}^{\theta \theta}}{\partial t} & =0 . \\
\frac{\partial \tilde{S}^{r z}}{\partial t} & =0 .
\end{aligned}
$$

Assim, podemos resolver (1.50) - (1.53) para as componentes $\tilde{S}^{r r}, \tilde{S}^{z z}, \hat{S}^{\theta \theta}$ e $\tilde{S}^{r z}$. De $(1.52)$ e (1.53) com a condição inicial $\mathbf{S}=\mathbf{0}$, temos

$$
S^{\theta \theta}(t+\delta t)=0 \quad \text { e } \quad S^{r z}(t+\delta t)=0 .
$$

$\Lambda$ equação (1.50) integrada no intervalo $[t, t+\delta t]$ fornecc

$$
\tilde{S}^{r r}(t+\delta t)-\tilde{S}^{r r}(t)=-\int_{t}^{t+\delta t} \frac{\partial\left(v \tilde{S}^{r r}\right)}{\partial z} d s+2 \int_{t}^{t+\delta t} \frac{\partial u}{\partial r} \tilde{S}^{r r} d s+\frac{1}{W e} \frac{2}{R e}\left(1-\frac{\lambda_{2}}{\lambda_{1}}\right) \int_{t}^{t+\delta t} e^{\frac{1}{W e} s} \frac{\partial u}{\partial r} d s
$$

Para a última integral da equação (1.55) aplicamos o teorema do valor médio para integrais e obtemos

$$
\tilde{S}^{r r}(t+\delta t)-\tilde{S}^{r r}(t)=-\int_{t}^{++\delta t} \frac{\partial\left(v \tilde{S}^{r r}\right)}{\partial z} d s+2 \int_{t}^{t+\delta t} \frac{\partial u}{\partial r} \tilde{S}^{r r} d s+\left.\frac{2}{R e}\left(1-\frac{\lambda_{2}}{\lambda_{1}}\right) \frac{\partial u}{\partial r}\right|_{t^{*}} e^{\frac{1}{W_{t}} t}\left[e^{\frac{1}{w_{e}} \delta t}-1\right]
$$


onde $\frac{\partial u}{\partial r}$ é avaliada em $t^{*} \in[t, t+\delta t]$. Aproximando as integrais da equação (1.56) pela regra do Trapézio, obtcmos

$$
\begin{aligned}
\tilde{S}^{r r}(l+\delta t)-\tilde{S}^{r r}(t) & =-\frac{\delta t}{2}\left(\left.\frac{\partial\left(v \tilde{S}^{r r}\right)}{\partial z}\right|_{t+\delta t}+\left.\frac{\partial\left(\nu \tilde{S}^{r r}\right)}{\partial z}\right|_{t}\right)+\delta t\left(\left.\left.\frac{\partial u}{\partial r}\right|_{t+\delta t} \tilde{S}^{r r}\right|_{t+\delta t}\right. \\
& \left.+\left.\left.\frac{\partial u}{\partial r}\right|_{t} \tilde{S}^{r r}\right|_{t}\right)+\left.\frac{2}{R e}\left(1-\frac{\lambda_{2}}{\lambda_{1}}\right) \frac{\partial u}{\partial r}\right|_{t^{*}} e^{\frac{1}{W \epsilon} t}\left[e^{\frac{1}{W e} \delta t}-1\right] .
\end{aligned}
$$

Introduzindo a mudança de variável $S^{r r}(t+\delta t)=e^{\frac{-1}{W e}(t+\delta t)} \tilde{S}^{r r}(t+\delta t)$ na equação (1.57), temos

$$
\begin{aligned}
S^{r r}(t+\delta t)=e^{\frac{1}{W e} \delta t} S^{r r}(t) & -\frac{\delta t}{2}\left(\left.\frac{\partial v S^{r r}}{\partial z}\right|_{t+\delta t}+\left.e^{\frac{-1}{W e} \delta t} \frac{\partial v S^{r r}}{\partial z}\right|_{t}\right)+\delta t\left(\left.\left.\frac{\partial u}{\partial r}\right|_{t+-\delta t} S^{r r}\right|_{t+\delta t}\right. \\
& \left.+\left.\left.e^{\frac{-1}{W e} \delta t} \frac{\partial u}{\partial r}\right|_{t} S^{r r}\right|_{t}\right)+\left.\frac{2}{R e}\left(1-\frac{\lambda_{2}}{\lambda_{1}}\right) \frac{\partial u}{\partial r}\right|_{t^{*}}\left[1-e^{\frac{1}{W r} \delta t}\right]
\end{aligned}
$$

De (1.58) obtemos o valor da componente não-newtoniana $S^{r r}$ do tensor extra-tcrsãa. Proccdendo de maneira análoga para a componente $S^{z z}$, a equação (1.51) fornece

$$
\begin{aligned}
S^{z z}(t+\delta t)=e^{\frac{1}{W_{e}} \partial t} S^{z z}(t) & -\frac{\delta \iota}{2}\left(\left.\frac{\partial v S^{z z}}{\partial z}\right|_{t+\delta t}+\left.e^{\frac{-1}{W e} \delta t} \frac{\partial v S^{z z}}{\partial z}\right|_{\ell}\right)+\delta l\left(\left.\left.\frac{\partial v}{\partial z}\right|_{t+\delta t} S^{z z}\right|_{t+\delta t}\right. \\
& \left.+\left.\left.e^{\frac{-1}{W_{c}} \delta l} \frac{\partial v}{\partial z}\right|_{t} S^{z z}\right|_{t}\right)+\left.\frac{2}{R e}\left(1-\frac{\lambda_{2}}{\lambda_{1}}\right) \frac{\partial v}{\partial z}\right|_{t^{*}}\left[1-e^{\frac{-1}{W e} \delta t}\right] .(1.59)
\end{aligned}
$$

As discretizações para o cálculo de $S^{r r}$ e $S^{z z}$ são feitas na seção 3.10 .

\subsubsection{Cálculo do Tensor extra-tensão em 'Inflows' e 'Outflows'}

Nestes contornos as componentes do tensor extra-tensão são especificados como segue.

\section{Contorno de Injeção - 'Inflow'}

Na entrada do fluido, isto é, no injetor, adotaremos a cstratégia empregada por Crochet (Crochet \& Walters [13]) e Mompean (Mompean e Deville [15]), em que considera-se que as componentes do tensor são dadas por

$$
S^{r r}=0, \quad S^{z z}=0, \quad S^{\theta 0}=0, \quad \text { e } \quad S^{r z}=0 .
$$

\section{Contorno de Ejeção - 'Outflow'}

Na saída do fluido, isto é, no ejetor, consideramos que não há variação da velocidade e variações das componentes do tensor na direção normal ao contorno. Para isso, impõem-se a condição homogênea de Neumann tanto para as componentes da velocidade dadas $\mathrm{cm}$ 
(1.29) quanto para as componentes do tensor extra-tensão $\mathbf{S}$ (ver (Mompean e Deville [15]) e (Phillips e Williams [18])), ou seja,

$$
\frac{\partial S^{r r}}{\partial n}=\frac{\partial S^{z z}}{\partial n}=\frac{\partial S^{\theta \theta}}{\partial n}=\frac{\partial S^{r z}}{\partial n}=0 .
$$

\subsubsection{Condições de Contorno na Superfície Livre}

Neste trabalho vamos considerar escoamentos transientes com superfícies livres de um fluido viscoelástico movendo-se dentro de uma atmosfera passiva, na qual pode-se tomar a pressão como sendo nula. Se desprezarmos as tensões superficiais, as condições de contorno na superfícic livre são (ver Batchelor [14])

$$
\begin{aligned}
& \mathbf{n} \cdot(\boldsymbol{\sigma} \cdot \mathbf{n})=0, \\
& \mathbf{m} \cdot(\boldsymbol{\sigma} \cdot \mathbf{n})=0,
\end{aligned}
$$

onde $\mathbf{n}$ é o vetor unitário normal exterior à superfície livre, $\mathbf{m}$ é o vetor tangencial a superfície livre e $\boldsymbol{\sigma}$ é o tensor de tensão total dado pcla equação (1.2)

$$
\sigma=-p \mathbf{I}+\tau,
$$

onde

$$
\tau=2 \mu_{0}\left(\frac{\lambda_{2}}{\lambda_{1}}\right) \mathbf{D}+\mathbf{S}
$$

que na forma adimensional é dado por

$$
\tau=\frac{2}{R e}\left(\frac{\lambda_{2}}{\lambda_{1}}\right) \mathbf{D}+\mathbf{S} .
$$

Tomando $\mathbf{n}=\left(n_{r}, n_{z}\right)$ e $\mathrm{m}=\left(n_{z:}-n_{r}\right)$ e utilizando (1.2) e (1.7), as equaçōes (1.62) e (1.63) se reduzem a

$$
\begin{aligned}
& -p+\frac{2}{\operatorname{Re}}\left[\left(\frac{\lambda_{2}}{\lambda_{1}}\right)\left(\frac{\partial u}{\partial r} n_{r}^{2}+\left(\frac{\partial u}{\partial z}+\frac{\partial v}{\partial r}\right) n_{z} n_{r}+\frac{\partial v}{\partial z} n_{z}^{2}\right)\right]+S^{r r} n_{r}^{2}+2 S^{z r} n_{r} n_{z}+S^{z z} n_{z}^{2}=0 \\
& \frac{1}{R e}\left(\frac{\lambda_{2}}{\lambda_{1}}\right)\left[2\left(\frac{\partial v}{\partial z}-\frac{\partial u}{\partial r}\right) n_{r} n_{z}+\left(\frac{\partial u}{\partial z}+\frac{\partial v}{\partial r}\right)\left(n_{r}^{2}-n_{z}^{2}\right)\right]+\left(S^{z z}-S^{r r}\right) n_{r} n_{z}+S^{z r}\left(n_{r}^{2}-n_{z}^{2}\right)=0
\end{aligned}
$$

respectivamente.

As equaçōes (1.64) e (1.65) constituem as condiçóes de contorno a serem impostas na supcrfície livre do fluido. A discretização dessas equaçõos por diferenças finitas são apresentadas na seção 3.8 . 


\section{Capítulo 2}

\section{Método Numérico}

Neste capítulo apresentaremos um método numérico para resolver as equações governantes juntamente com as condições de contorno e iniciais. O método numérico desenvolvido é baseado no trabalho apresentado por Tomé et al. [12] para escoamentos viscoelásticos bidimensionais com superfícies livres.

\subsection{Algoritmo Computacional}

Para resolvermos as equações (1.20) - (1.26) suponhamos que em um dado tempo $t_{n}$, o vetor velocidade $\mathbf{u}\left(r, z, t_{n}\right)$ e o tensor extra-tensão $\mathbf{S}\left(r, z, l_{n}\right)$ são conhecidos. Além disso, são dadas as condiçōes de contorno para o vetor velocidade $\mathbf{u}$, o tensor extra-tensão $\mathbf{S}$ e a pressão.

Então, para calcular o vetor velocidade, o campo de pressão e o tensor extra-tensão no tempo atual $t_{n+1}=t_{n}+\delta t$ procedemos da seguinte maneira:

Passo 1: Scja $\tilde{p}\left(r, z, \iota_{n}\right)$ um campo de pressão arbitrário que satisfaz as condições de contorno para a pressão na superfície livre. Este campo de pressão é calculado por meio da equação (1.64).

Passo 2: Calcular o vetor velocidade intermediário, $\tilde{\mathbf{u}}\left(r, z, t_{n+1}\right)$ por

$$
\begin{aligned}
\frac{\partial \tilde{u}}{\partial t}= & -\frac{1}{r} \frac{\partial\left(r u^{2}\right)}{\partial r}-\frac{\partial(u v)}{\partial z}-\frac{\partial \tilde{p}}{\partial r}+\frac{1}{R e}\left(\frac{\lambda_{2}}{\lambda_{1}}\right)\left[\frac{1}{r} \frac{\partial}{\partial r}\left(r \frac{\partial u}{\partial r}\right)+\frac{\partial^{2} u}{\partial z^{2}}-\frac{u}{r^{2}}\right] \\
& +\left(\frac{1}{r} \frac{\partial}{\partial r}\left(r S^{r r}\right)+\frac{\partial S^{r r}}{\partial z}-\frac{S^{\theta \theta}}{r}\right)+\frac{1}{F_{r}^{2}} g_{r} \\
\frac{\partial \tilde{v}}{\partial t}= & -\frac{1}{r} \frac{\partial(r u v)}{\partial r}-\frac{\partial\left(v^{2}\right)}{\partial z}-\frac{\partial \tilde{p}}{\partial z}+\frac{1}{R e}\left(\frac{\lambda_{2}}{\lambda_{1}}\right)\left[\frac{1}{r} \frac{\partial}{\partial r}\left(r \frac{\partial v}{\partial r}\right)+\frac{\partial^{2} v}{\partial z^{2}}\right] \\
& +\left(\frac{1}{r} \frac{\partial}{\partial r}\left(r S^{r z}\right)+\frac{\partial S^{z z}}{\partial z}\right)+\frac{1}{F_{r}^{2}} g_{z} .
\end{aligned}
$$


Passo 3: Resolver a equação de Poisson

$$
\nabla^{2} \psi\left(r, z, l_{n+1}\right)=\nabla \cdot \tilde{\mathbf{u}}\left(r, z ; l_{n+1}\right),
$$

sujeita as seguintes condições de frontcira (ver (Tomé et al. [12]))

$$
\frac{\partial \psi\left(r, z, \iota_{n+1}\right)}{\partial n}=0
$$

cm contornos rígidos e 'inflows', e

$$
\psi=0
$$

na superfície livre e 'outflows', sendo $n$ a direção normal ao contorno rígido.

Passo 4: Calcular o vetor velocidade final por

$$
\mathbf{u}\left(r, z, t_{n+1}\right)=\tilde{\mathbf{u}}\left(r, z, t_{n+1}\right)-\nabla \psi\left(r, z, t_{n+1}\right) .
$$

Passo 5: Calcular a pressão. Pode-sc mostrar que a pressão é dada por (ver Tomé et al. [1])

$$
p\left(r, z, t_{n+1}\right)=\tilde{p}\left(r, z, t_{n}\right)+\frac{\psi\left(r, z, t_{n+1}\right)}{\delta t} .
$$

Passo 6: Atualizar as componentes do tensor extra-tensão nos contornos rígidos por meio da s equações derivadas na seção 1.2.1.

Passo 7: Atualizar as componentes do tensor extra-tensão no cixo de simetria por meio das cquações derivadas na seção 1.2.2.

Passo 8: Calcular as componentes do tensor extra-tensão $S^{r r}\left(r, z, t_{n+1}\right), S^{\theta \theta}\left(r, z, t_{n+1}\right)$, $S^{r z}\left(r, z, \iota_{n+1}\right) \subset S^{z z}\left(r, z, \iota_{n+1}\right)$ por meio das equações

$$
\begin{aligned}
\frac{\partial S^{r r}}{\partial t}= & {\left[-\frac{1}{r} \frac{\partial\left(r u S^{r r}\right)}{\partial r} \cdot \frac{\partial\left(v S^{r r}\right)}{\partial z}+2 \frac{\partial u}{\partial r} S^{r r}+2 \frac{\partial u}{\partial z} S^{r z}\right.} \\
+ & \left.\frac{1}{W e}\left[\frac{2}{R e}\left(1-\frac{\lambda_{2}}{\lambda_{1}}\right) \frac{\partial u}{\partial r}-S^{r r}\right]\right]_{t_{n}} \\
\frac{\partial S^{\theta \theta}}{\partial t}= & {\left[-\frac{1}{r} \frac{\partial\left(r u S^{\theta \theta}\right)}{\partial r}-\frac{\partial\left(v S^{\theta \theta}\right)}{\partial z}+2 \frac{u}{r} S^{\theta \theta}\right.} \\
& \left.+\frac{1}{W e}\left[\frac{2}{\operatorname{Re}}\left(1-\frac{\lambda_{2}}{\lambda_{1}}\right) \frac{u}{r}-S^{\theta \theta}\right]\right]_{t_{n}}, \\
\frac{\partial S^{\prime z z}}{\partial t}= & {\left[-\frac{1}{r} \frac{\partial\left(r u S^{z z}\right)}{\partial r}-\frac{\partial\left(v S^{S^{z} z}\right)}{\partial z}+2 \frac{\partial v}{\partial r} S^{r z}+2 \frac{\partial v}{\partial z} S^{r z}\right.} \\
+ & \left.\frac{1}{W e}\left[\frac{2}{R e}\left(1-\frac{\lambda_{2}}{\lambda_{1}}\right) \frac{\partial v}{\partial z}-S^{r z}\right]\right]_{t_{n}}
\end{aligned}
$$




$$
\begin{gathered}
\frac{\partial S^{r z}}{\partial t} \quad\left[-\frac{1}{r} \frac{\partial\left(r u S^{r z}\right)}{\partial r}-\frac{\partial\left(v S^{r z}\right)}{\partial z}+\frac{\partial v}{\partial r} S^{r r}+\frac{\partial u}{\partial z} S^{z z}-\frac{u}{r} S^{r z}\right. \\
\left.+\frac{1}{W e}\left[\frac{1}{R e}\left(1-\frac{\lambda_{2}}{\lambda_{1}}\right)\left(\frac{\partial u}{\partial z}+\frac{\partial v}{\partial r}\right)-S^{r z}\right]\right]_{t_{n}} .
\end{gathered}
$$

Passo 9: Atualizar as posições das partículas marcadoras que representam o fluido. Estas partículas são utilizadas para representar a superfície livre do fluido cujas coordenadas são armazenadas e atualizadas no fim de cada ciclo de cálculo integrando as equações

$$
\frac{d r}{d t}=u, \quad \frac{d z}{d t}=v,
$$

obtendo assim a ııova posição para cada partícula marcadora

As equações correspondentes aos passos Passo 1 - Passo 5 são análogas às cquações correspondentes a fluidos ncwtonianos e já são resolvidas pelo sistema de simulação FreeflowAXI. Neste trabalho precisamos desenvolver equações de diferenças finitas para resolver as equaçôes provenientes dos passos Passo 6, Passo 7 e Passo 8, conforme será apresentado no capítulo 3 a seguir. 


\section{Capítulo 3}

\section{Aproximação por Diferenças Finitas}

Este capítulo apresenta as discretizações para a resolução das equações do método numérico apresentado no capítulo 2. As cquações (2.1) - (2.9) serão resolvidas pclo método de diferenças finitas sobre uma malha deslocada, com espaçamento $\delta r$ e $\delta z$ nas direções $r$ c $z$, respectivamente.

As componentes da velocidade $u$ e $v$ são aproximadas nos pontos $\left(i+\frac{1}{2}, j\right)$ e $\left(i, j+\frac{1}{2}\right)$, respectivamente. No centro da célula $(i, j)$ são avaliadas a pressão, a função potencial $\psi \mathrm{e}$ as componentes $S^{r r}, S^{\theta \theta}, S^{r z}$ e $S^{z z}$ do tensor extra-tensão $\mathrm{S}$ (ver figura 3.1).

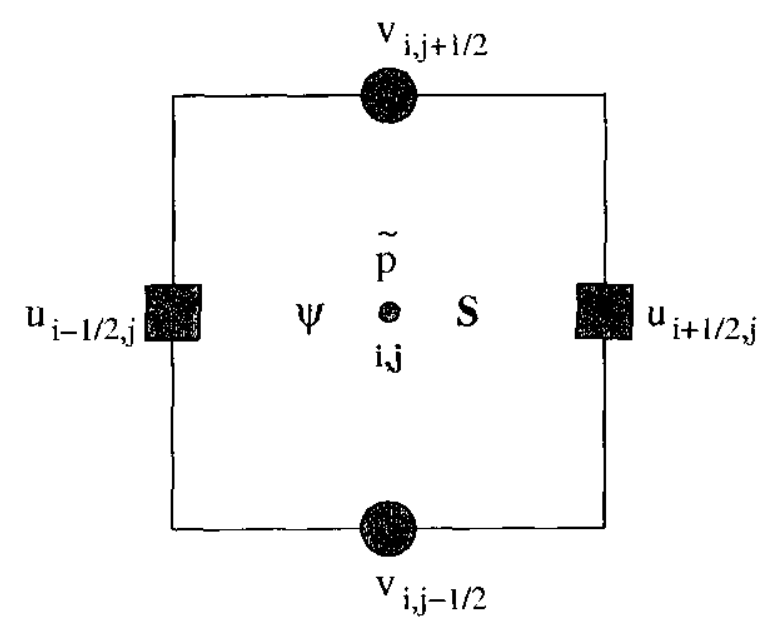

Figura 3.1: Célula Computacional.

\subsection{Classificação das Células}

Como o fluido está continuamente em movimento, é necessário fazer uma classificação das células da malha para identificar se as mesmas contém ou não fluido, se estão no contorno rígido ou são cêlulas que contém a superfície livre. Para tanto, adota-se a seguinte 
classificação:

- Células vazias (E): São células que não contém fluido;

- Células cheias (F): São células cheias de fluido e não possuem nenhuma face em contato com células vazias;

- Células de superfície (S): São células que contém fluido e possuem pelo menos uma face em contato com células vazias;

- Células de contorno (B): São células que definem um contorno rígido de maneira que as condições de contorno possam ser impostas;

- Células 'Inflow' (I): São células que definem a entrada de fluido;

- Células 'Outflow' (O): São células que definem a saída de fluido.

A figura 3.2 ilustra a estrutura de células na malha em um dado instante.

\begin{tabular}{|c|c|c|c|c|}
\hline 0 & o & $\mathrm{o}$ & 0 & 0 \\
\hline E & E & E & E & E \\
\hline E & E & E & E & E \\
\hline $\mathrm{E}$ & $\mathrm{E}$ & E & E & $\mathrm{E}$ \\
\hline$\hat{s}$ & $\mathbf{s}$ & $\mathrm{E}$ & E & $\mathrm{E}$ \\
\hline $\mathbf{F}$ & $\mathrm{F}$ & $s$ & E & $\mathrm{E}$ \\
\hline $\mathbf{F}$ & $\mathrm{F}$ & $\$$ & E & E \\
\hline $\mathbf{F}$ & $\mathrm{F}$ & s & E & E \\
\hline $\mathbf{F}$ & $\mathrm{F}$ & $s$ & E & $\mathrm{E}$ \\
\hline $\mathbf{F}$ & F & B & B & B \\
\hline $\mathbf{F}$ & $\mathbf{F}$ & B & & \\
\hline $\mathbf{F}$ & $\mathbf{F}$ & B & & \\
\hline I & I & & & \\
\hline
\end{tabular}

Figura 3.2: Tipos de célula no domínio computacional.

\subsection{Discretização das Equações}

Para o cálculo das componentes da velocidade $\tilde{u}, \tilde{v}$ das equações (2.1) e (2.2), as derivadas temporais são aproximadas pelo método de Euler Explícito. O gradiente de pressão é aproxi -mado por diferenças centrais e para os termos convectivos utilizamos o método 'upwind' 
de alta ordem CLBISTA [16]. Os tcrmos envolvendo o divergente do tensor extra- tensão da equação (2.1) são aproximados por diferenças centrais como

$$
\begin{gathered}
\left.\frac{1}{r} \frac{\partial}{\partial r}\left(r S^{r r}\right)\right|_{i+\frac{1}{2}, j}=\frac{1}{r_{i+\frac{1}{2}}} \frac{\left(r_{i+1} S_{i+1, j}^{r r}-r_{i} S_{i, j}^{r r}\right)}{\delta r}, \\
\left.\frac{\partial S^{S r z}}{\partial z}\right|_{i+\frac{1}{2}, j}=\frac{S_{i+\frac{1}{2}, j+\frac{1}{2}}^{r z}-S_{i+\frac{1}{2}, j-\frac{1}{2}}^{r z}}{\delta z}
\end{gathered}
$$

onde os termos $S_{i \vdash \cdot \frac{1}{2}, j+\frac{1}{2}}^{r z}$ são aproximados por uma média aritmética dos quatro valores mais próximos, isto é,

$$
S_{i+\frac{1}{2}, j+\frac{1}{2}}^{r z} \frac{\left(S_{i, j}^{r z}+S_{i+1, j}^{r z}+S_{i, j+1}^{r z}+S_{i \nmid 1, j+1}^{r z}\right)}{4} .
$$

Se a célula $(i, j)$ faz vizinhança corn uma cólula de contorno pela face supcrior ou pela face inferior, aproximna-se a derivada $\left.\frac{\partial S^{r z}}{\partial z}\right|_{i, \frac{1}{2}, j}$ por diferenças progressivas ou regressivas (ver Tomé et al. [12]). Neste trabalho, utilizaremos uma expansão en sćric de Taylor para $S^{r z}$ no centro da célula $(i, j)$ para obtermos uma aproximação de $2^{\circ}$ ordem para csta derivada. No caso da figura $3.3 \mathrm{a} \mathrm{cm}$ que célula $(i, j)$ tem a face superior em contato com uma cólula de contorno (B), utilizando série de Taylor, obtém-se a seguinte aproximação de $2^{\circ}$ ordem para $\frac{\partial S^{r z}}{\partial z}$

$$
\left.\frac{\partial S^{r z}}{\partial z}\right|_{i+\frac{1}{2}, j}=\frac{3 S_{i+\frac{1}{2}, j}^{r z}-4 S_{i+\frac{1}{2}, j}^{r z}+S_{i+\frac{1}{2}, j-2}^{r z}}{2 \delta z},
$$

onde os termos do (3.4) são aproximados por médias aritméticas

$$
S_{i+\frac{1}{2}, j \cdots 2}^{r z}:-\frac{S_{i, j-2}^{r z}+S_{i+1, j-2}^{r z}}{2}, \quad S_{i+\frac{1}{2}, j-1}^{r z}=\frac{S_{i, j-1}^{r z}+S_{i+1, j}^{r z}}{2}, \quad S_{i+\frac{1}{2}, j}^{r z}=\frac{S_{i, j}^{r z}+S_{i+1, j}^{r z}}{2} .
$$

No caso em que a célula $(i, j)$ tem a face inferior em contato com uma célula de contorno (B), procedemos da mesina forma.

Para aproximar a derivada $\left.\frac{1}{r} \frac{\partial}{\partial r}\left(r S^{r z}\right)\right|_{i, j+\frac{1}{2}}$ da equação $(2.2)$ procedemos de maneira semclhante, como segue (ver figura 3.3b)

$$
\left.\frac{1}{r} \frac{\partial}{\partial r}\left(r S^{r z}\right)\right|_{i, j+\frac{1}{2}}-\frac{1}{r_{i}} \frac{\left(r_{i-2} S_{i}^{T_{2} \tau, j+\frac{1}{2}}-4 r_{i \cdot 1} S_{i-1, j+\frac{1}{2}}^{r z}+3 r_{i} S_{i, j+\frac{1}{2}}^{r z}\right)}{2 \delta r},
$$

onde

$$
S_{i-2, j+\frac{1}{2}}^{r z}-\frac{S_{i-2, j}^{r z}+S_{i-2, j+1}^{r z}}{2}, \quad S_{i-1, j+\frac{1}{2}}^{r z}=\frac{S_{i-1, j}^{r z}+S_{i-1, j ! 1}^{r z}}{2}, \quad S_{i, j+\frac{1}{2}}^{r z}=\frac{S_{i, j}^{r z}+S_{i, j+1}^{r z}}{2},
$$




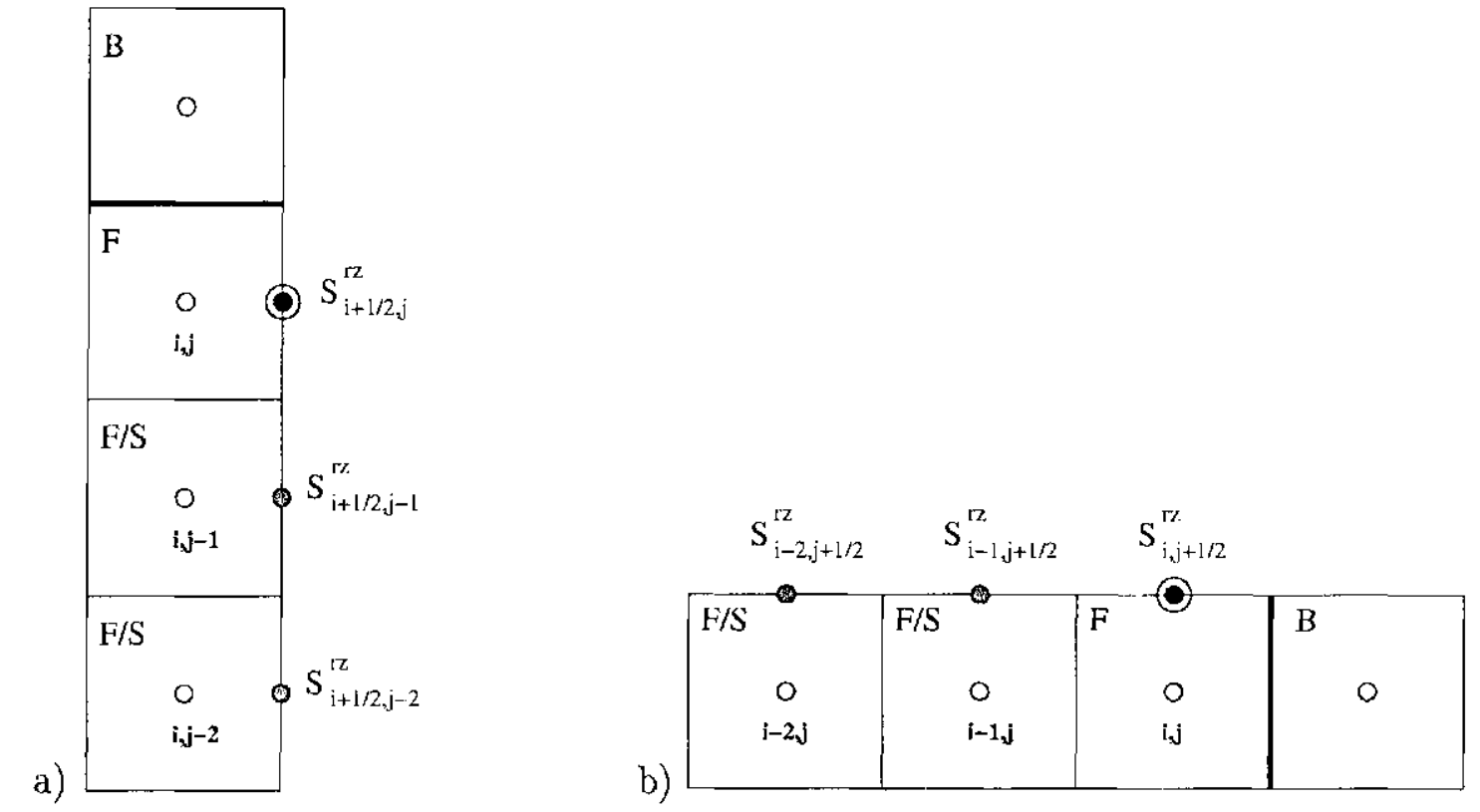

Figura 3.3: $\Lambda$ proximação de $\frac{\partial S^{r z}}{\partial z}$ e $\frac{1}{r} \frac{\partial}{\partial r}\left(r S^{r z}\right)$ cm pontos adjacentes ao contorno rígido.

Para calcularmos as equações discretizadas $(2.1)$ e (2.2) procedemos da seguinte forma

$$
\begin{aligned}
& u_{i+\frac{1}{2}, j}^{(n+1)}=u_{i+\frac{1}{2}, j}+\delta t F(u, v), \\
& v_{i, j+\frac{1}{2}}^{(n+1)}=v_{i, j+\frac{1}{2}}+\delta t G(u, v),
\end{aligned}
$$

onde as funções $F(u, v), G(u, v)$ podem ser escritas como

$$
\begin{aligned}
F(u, v)= & -\frac{1}{r_{i+\frac{1}{2}}} \operatorname{conv}\left(r u^{2}\right)-\operatorname{conv}(v u)-\frac{\tilde{p}_{i+1, j}-\tilde{p}_{i, j}}{\delta r} \\
& +\frac{1}{R e}\left(\frac{\lambda_{2}}{\lambda_{1}}\right)\left[\frac{1}{r_{i+\frac{1}{2}}} \frac{\left(r_{i} u_{i \cdots \frac{1}{2}, j}-\left(r_{i}+r_{i+1}\right) u_{i+\frac{1}{2}, j}+r_{i+1} u_{i+\frac{3}{2}, j}\right)}{\delta r^{2}}\right. \\
& \left.+\frac{\left(u_{i+\frac{1}{2}, j-1}-2 u_{i+\frac{1}{2}, j}+u_{i+\frac{1}{2}, j+1}\right)}{\delta z^{2}}-\frac{u_{i+\frac{1}{2}, j}}{r_{i+\frac{1}{2}}^{2}}\right] \\
& +\left(\frac{1}{r_{i+\frac{1}{2}}} \frac{r_{i+1} S_{i+1, j}^{r r}-r_{i} S_{i, j}^{r r}}{\delta r}+\frac{S_{i+\frac{1}{2}, j+\frac{1}{2}}^{r z}-S_{i+\frac{1}{2}, j-\frac{1}{2}}^{r z}}{\delta z}+\frac{S_{i+1, j}^{\theta \theta}+S_{i, j}^{\theta \theta}}{2 r_{i+\frac{1}{2}}}\right)+\frac{1}{F_{r}^{2}} g_{r},(3.9)
\end{aligned}
$$




$$
\begin{aligned}
G(u, v) & =-\frac{1}{r_{i}} \operatorname{conv}(r u v)-\operatorname{conv}\left(v^{2}\right)-\frac{\bar{p}_{i, j+1}-\hat{p}_{i, j}}{\delta z} \\
& +\frac{1}{R e}\left(\frac{\lambda_{2}}{\lambda_{1}}\right)\left[\frac{1}{r_{i}} \frac{\left(r_{i-\frac{1}{2}} v_{i-1, j+\frac{1}{2}}-\left(r_{i+\frac{1}{2}}+r_{i \frac{1}{2}}\right) v_{i, j+\frac{1}{2}}+r_{i 1 \frac{1}{2}} v_{i \nmid 1, j+\frac{1}{2}}\right)}{\delta r^{2}}\right. \\
& \left.+\frac{\left(v_{i, j-\frac{1}{2}}-2 v_{i, j+\frac{1}{2}}+v_{i, j+\frac{3}{2}}\right)}{\delta z^{2}}\right] \\
& +\left(\frac{1}{r_{i}} \frac{\left.r_{i \mid \frac{1}{2}} S_{i+\frac{1}{2}, j+\frac{1}{2}}^{T r}-r_{i} \frac{1}{2} S_{i-\frac{1}{2}, j+\frac{1}{2}}^{T z}+\frac{S_{i, j+1}^{z z}-S_{i, j}^{z z}}{\delta z}\right)+\frac{1}{F_{r}^{2}} g_{z}}{\delta r}\right.
\end{aligned}
$$

onde $\operatorname{conv}\left(r u^{2}\right), \operatorname{conv}(v u), \operatorname{conv}(r u v)$ e conv $\left(v^{2}\right)$ são as discretizações dos termos convectivos que serão apresentadas na seção 3.7 .

Para a discretização das equações das componentes $S^{r r}, S^{\theta \theta}, S^{r z}$ e $S^{z z}$ do tensor extratensão procedemos da mesma forma que foi feito para as equações das componentes da velocidade. Novamente, para tratar os termos convectivos utilizamos o esquema CUBIST $\Lambda$ [16] e o método de Euler Explícito para aproximar as derivadas temporais. Assim, as equações (2.6) - (2.9) podem ser escritas como

$$
\begin{aligned}
& S_{i, j}^{r r^{(n+1)}}=S_{i, j}^{r r}+\delta t H_{1}\left(S^{r r}, S^{r z}, S^{\theta \theta}, S^{z z}\right), \\
& S_{i, j}^{r^{(n+1)}}=S_{i, j}^{r z}+\delta t H_{2}\left(S^{r r}, S^{r z}, S^{\theta \theta}, S^{r z}\right), \\
& S_{i, j}^{\theta \theta(n+1)}=S_{i, j}^{\theta \theta}+\delta t H_{3}\left(S^{r r}, S^{r z}, S^{\theta \theta}, S^{r z}\right), \\
& S_{i, j}^{z^{\left(z^{(n+1)}\right.}}=S_{i, j}^{z z z}+\delta t H_{4}\left(S^{r r}, S^{r z}, S^{\theta \theta}, S^{z z}\right),
\end{aligned}
$$

onde as funções $H_{1}, H_{2}, H_{3}$ e $H_{4}$ podem ser escritas como

$$
\begin{aligned}
H_{1} \quad & -\frac{1}{W e} S_{i, j}^{r \tau}-\frac{1}{r_{i}} \operatorname{conv}\left(r u S^{r r}\right)_{i, j}-\operatorname{conv}\left(v S^{r r}\right)_{i, j} \mid 2 \frac{\left(u_{i+\frac{1}{2}, j}-u_{i-\frac{1}{2}, j}\right)}{\delta r} S_{i, j}^{r r} \\
+ & 2 \frac{\left(\left.u_{i, j}\right|_{2} ^{1}-u_{i, j-\frac{1}{2}}\right)}{\delta z} S_{i, j}^{r z}+\frac{1}{R e} \frac{2}{W e}\left(1-\frac{\lambda_{2}}{\lambda_{1}}\right) \frac{\left(u_{i+\frac{1}{2}, j}-u_{i-\frac{1}{2}, j}\right)}{\delta r}, \\
H_{2}= & -\frac{1}{W e} S_{i, j}^{z z}-\frac{1}{r_{i}} \operatorname{conv}\left(r u S^{z z}\right)_{i, j}-\operatorname{conv}\left(v S^{z z}\right)_{i, j}+2 \frac{\left(v_{i, j+\frac{1}{2}}-v_{i, j} \frac{1}{2}\right)}{\delta z} S_{i, j}^{z z} \\
+ & 2 \frac{\left(v_{i+\frac{1}{2}, j}-v_{i \cdot \frac{1}{2}, j}\right)}{\delta r} S_{i, j}^{r z}+\frac{1}{R e} \frac{2}{W e}\left(1-\frac{\lambda_{2}}{\lambda_{1}}\right) \frac{\left(v_{i, j+\frac{1}{2}}-v_{i, j-\frac{1}{2}}\right)}{\delta z},
\end{aligned}
$$




$$
\begin{aligned}
& I_{3}=-\frac{1}{W_{e}} S_{i, j}^{\theta \theta}-\frac{1}{r_{i}} \operatorname{conv}\left(r u S^{\theta \theta}\right)_{i, j}-\operatorname{conv}\left(v S^{0 \theta}\right)_{i, j}+\frac{1}{r_{i}}\left(u_{i+\frac{1}{2}, j}+u_{i-\frac{1}{2}, j}\right) S_{i, j}^{\theta \theta} \\
& +\frac{1}{\operatorname{Re}} \frac{2}{W e}\left(1-\frac{\lambda_{2}}{\lambda_{1}}\right) \frac{\left(u_{i+\frac{1}{2}, j}+u_{i \cdot \frac{1}{2}, j}\right)}{2 r_{i}} \\
& I_{4} \quad-\frac{1}{W_{e}} S_{i, j}^{r z}-\frac{1}{r_{i}} \operatorname{conv}\left(r u S^{r z}\right)_{i, j}-\operatorname{conv}\left(v S^{r z}\right)_{i, j}+\frac{\left(v_{i+\frac{1}{2}, j}-v_{i-\frac{1}{2}, j}\right)}{\delta r} S_{i, j}^{r r} \\
& +\frac{\left(u_{i, j+\frac{1}{2}}-u_{i, j} \frac{1}{2}\right)}{\delta z} S_{i, j}^{z z}+\frac{1}{R e} \frac{1}{W e}\left(1-\frac{\lambda_{2}}{\lambda_{1}}\right)\left(\frac{u_{i, j+\frac{1}{2}}-u_{i, j-\frac{1}{2}}}{\delta z}+\frac{v_{i+\frac{1}{2}, j}-v_{i} \frac{1}{2}, j}{\delta r}\right)
\end{aligned}
$$

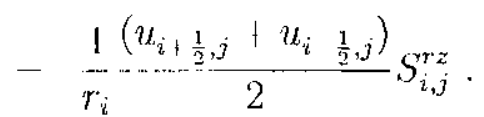

\subsection{Aproximação da Equação de Poisson para $\psi$}

$\Lambda$ cquação de Poisson (2.3) cm coordenadas cilíndricas é dada por

$$
\left.\frac{\partial}{\partial r}\left(r \frac{\partial \psi}{\partial r}\right)+r \frac{\partial^{2} \psi}{\partial z^{2}}=r \hat{l}\right)
$$

$\Lambda$ proximando as derivadas por diferenças finitas de $2^{\circ}$ ordem e assumindo $\delta r \quad \delta z$, temos

$$
-r_{i} \psi_{i, j \cdots 1}-r_{i}{ }_{2}^{1} \psi_{i \cdots 1, j}+4 r_{i} \psi_{i, j}-r_{i+\frac{1}{2}} \psi_{i+1, j}-r_{i} \psi_{i, j+1}--r_{i} \delta r^{2} \tilde{D}_{i, j}
$$

onde

$$
\tilde{D}_{i, j} \cdots \frac{1}{r_{i}}\left(\frac{r_{i+\frac{1}{2}} \tilde{u}_{i+\frac{1}{2}, j} \cdots r_{i-\frac{1}{2}} \tilde{u}_{i} \frac{1}{2}, j}{\delta r}\right)+\frac{\tilde{v}_{i, j \backslash \frac{1}{2}}-\tilde{v}_{i, j-\frac{1}{2}}}{\delta z} .
$$

$\Lambda$ cquação (3.16) quando aplicada em todos os pontos do domínio gera um sistema linear para $\psi_{i, j}$ cuja matriz ć simćtrica e definida positiva. Este sistema ć resolvido pelo método de gradicntes conjugados.

\subsection{Aproximação da Equação da Velocidade Final}

$\Lambda$ velocidado final ć obtida discretizando a equação (2.4) nas faces $\left(i+\frac{1}{2}, j\right) \subset\left(i, j \mid \frac{1}{2}\right)$, isto $\hat{c}$,

$$
\left\{\begin{array}{cc}
u_{i+\frac{1}{2}, j}^{n+1} & -\tilde{u}_{i+\frac{1}{2}, j}^{n+1}-\left(\frac{\psi_{i+1, j}^{n+1}-\psi_{i, j}^{n+1}}{\delta r}\right), \\
v_{i, j+\frac{1}{2}}^{n+1} & \cdots \tilde{v}_{i, j+\frac{1}{2}}^{n+1}-\left(\frac{\psi_{i, j+1}^{n+1}-\psi_{i, j}^{n+1}}{\delta z}\right) .
\end{array}\right.
$$




\subsection{Aproximação da Equação da Pressão}

$\Lambda$ pressão é obtida pela aplicação de $(2.5)$ no centro da célula $(i, j)$

$$
p_{i, j}^{n+1}=\tilde{p}_{i, j}+\frac{\psi_{i, j}^{n+1}}{\delta t} .
$$

\subsection{Cálculo do passo no tempo}

O tamanho do passo no tempo é calculado segundo as seguintes restrições:

$\left.1^{\circ}\right)$ Nenhuma partícula pode cruzar mais do quo uma célula cm um dado intervalo de tempo, ou scja,

$$
\delta t<\frac{\delta r}{|u|} \text { e } \delta t<\frac{\delta \tilde{z}}{|v|}
$$

$\left.2^{\circ}\right)$ Adotaremos a seguinte restrição de cstabilidade que envolve os números de Reynolds e Weissenberg por causa da discretização explícita da equação de quantidade de movimento

$$
\left\{\begin{aligned}
\delta t & =0.25 W e \text { We } \delta r^{2} \quad \text { se } \quad \begin{array}{c}
R e \\
W e
\end{array} \\
\delta t & =0.25 \delta r^{2} \quad \text { caso contrário }
\end{aligned}\right.
$$

Para satisfazer (3.18) é suficiente que

$$
\delta t<\frac{\delta r}{\left|U_{\max }\right|} \quad \text { ○ } \delta t<\frac{\delta z}{\left|V_{\max }\right|}
$$

onde $U_{\max } \mathrm{c} V_{\max }$ são os máximos valores de $u c v$.

A implementação dessas condições segue as mesmas normas como implementado no código GENSMAC (Tomé e McKeo |2|).

\subsection{Discretização dos Termos Convectivos}

Os termos convectivos são tratados de maneira particular por serem os responsáveis por muitos fenômenos complexos nos escoamentos e são os principais causadores de instabilidades numéricas nas simulações. Para estimarmos os termos convectivos das equações (1.21) - (1.26) utilizamos o esquema de alta ordem CLBISTA [16] (' $\Lambda$ convergent and universally bounded interpolation scheme for the treatment of advection').

Considere a figura 3.4 , onde $\phi_{A}$ e $\phi_{B}$ são os valores da variável genérica $\phi$ nos pontos $P_{A}$ e $P_{B}$, respectivamente. Para calcular a derivada parcial da variável genérica $\phi$ no ponto $P_{0}$, digamos $\frac{\partial \phi}{\partial s}$, onde $s$ é um dos eixos coordenados (neste caso, $r$ ou $z$ ), aproximamos por

$$
\left.\frac{\partial \phi}{\partial s}\right|_{P_{0}}-\frac{\phi_{B}-\phi_{A}}{\Delta s}
$$




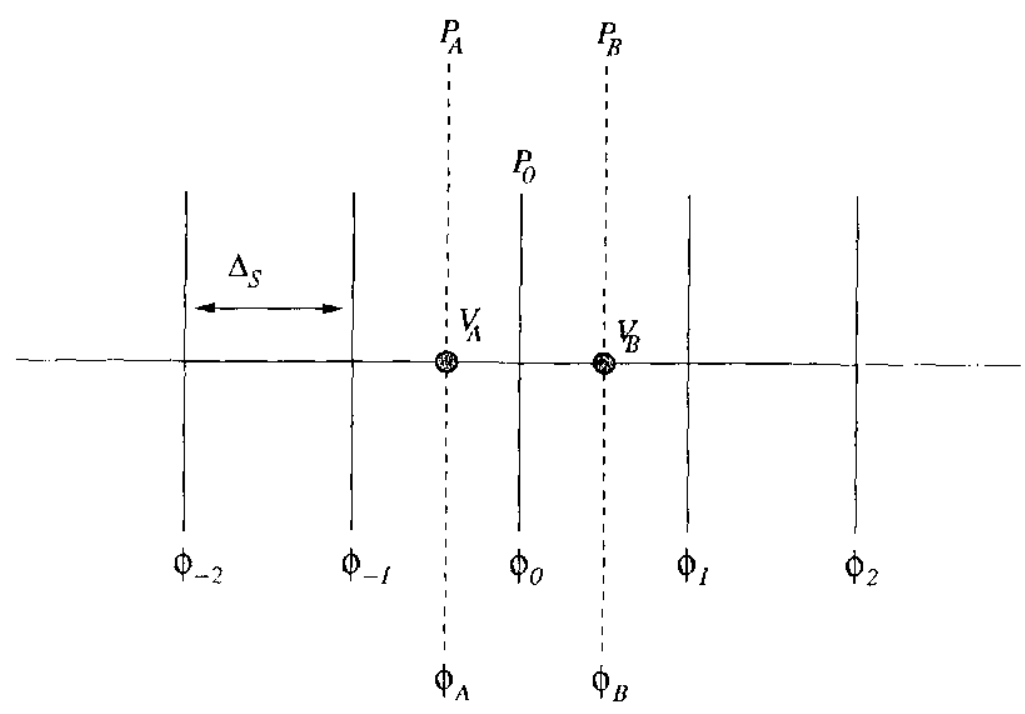

Figura 3.4: 'Stencil' usado para calcular $\phi_{A}$ e $\phi_{B}$ usando o esquema CUBISTA.

Os valores de $\phi_{A}$ e $\phi_{B}$ são obtidos em termos dos valores vizinhos, $\phi_{2}, \phi_{-1}, \phi_{0}, \phi_{1}, \phi_{2}$ e as direções das velocidades convectivas $\left(V_{A}, V_{B}\right)$, como scguc.

Esquema CUBISTA:

$$
\begin{aligned}
& \begin{cases}\phi_{0}, & \hat{\phi}_{0} \notin[0,1] \\
7 & -3,1,\end{cases} \\
& \text { se } V_{B} \geq 0, \quad \phi_{B}= \begin{cases}\frac{7}{4} \phi_{0}-\frac{3}{4} \phi 1, & \hat{\phi}_{0} \in(0,3 / 8) \\
3 & \left.\hat{\phi}_{0}, \frac{1}{3} \phi_{1}+3 / 3 / 4\right)\end{cases} \\
& \begin{cases}\frac{3}{8} \phi_{1}+\frac{6}{8} \phi_{0}-\frac{1}{8} \phi_{-1}, \quad \hat{\phi}_{0} \in[3 / 8,3 / 4] \\
3 \phi_{1}+\frac{1}{4} \phi_{0}\end{cases} \\
& \text { se } \quad V_{B}<0, \quad \phi_{B}= \begin{cases}\phi_{1}, & \hat{\phi}_{1} \notin[0,1] \\
\frac{7}{4} \phi_{1}-\frac{3}{4} \phi_{2}, & \hat{\phi}_{1} \in(0,3 / 8) \\
\frac{3}{8} \phi_{0}+\frac{6}{8} \phi_{1}-\frac{1}{8} \phi_{2}, & \hat{\phi}_{1} \in[3 / 8,3 / 4] \\
\frac{3}{4} \phi_{0}+\frac{1}{4} \phi_{1}, & \hat{\phi}_{1} \in(3 / 4,1)\end{cases} \\
& \text { se } \quad V_{A} \geq 0, \quad \phi_{A} \cdot \begin{cases}\phi_{-1}, & \hat{\phi}_{-1} \notin[0,1] \\
\frac{7}{4} \phi_{.1}-\frac{3}{4} \phi_{-2}, & \hat{\phi}_{-1} \in(0,3 / 8) \\
\frac{3}{8} \phi_{0}+\frac{6}{8} \phi_{-1}-\frac{1}{8} \phi_{-2}, & \hat{\phi}_{-1} \in[3 / 8,3 / 4] \\
\frac{3}{4} \phi_{0}+\frac{1}{4} \phi_{-1}, & \hat{\phi}_{-1} \in(3 / 4,1)\end{cases} \\
& \text { se } \quad V_{A}<0, \quad \phi_{A}= \begin{cases}\phi_{0}, & \left.\hat{\phi}_{0} \notin \mid 0,1\right] \\
\frac{3}{4} \phi_{0}-\frac{3}{4} \phi_{1}, & \hat{\phi}_{0} \in(0,3 / 8) \\
\frac{3}{8} \phi_{.1}+\frac{6}{8} \phi_{0}-\frac{1}{8} \phi_{1}, & \hat{\phi}_{0} \in[3 / 8,3 / 4] \\
\frac{3}{4} \phi_{\cdots 1}+\frac{1}{4} \phi_{0}, & \hat{\phi}_{0} \in(3 / 4,1)\end{cases}
\end{aligned}
$$

onde $\hat{\phi}_{i},(i=-1,0,1)$, é definido em função das velocidades 'upstream' $\left(\phi_{U}\right)$, 'remoteupstream' $\left(\phi_{R}\right)$ e 'downstream' $\left(\phi_{D}\right)$ no ponto $P_{A}$ e $P_{B}$, isto $\hat{e}$, de acordo com a dirç̧ão da 
velocidade convectiva e é definido por

$$
\hat{\phi}_{i} \quad \frac{\phi_{U}-\phi_{R}}{\phi_{l}-\phi_{R}} .
$$

Por exemplo, considere o termo convectivo $\operatorname{conv}\left(v^{2}\right) \quad \frac{\partial(v v)}{\partial z}$ que é avaliado no ponto $\left(i, j+\frac{1}{2}\right)$. A derivada é aproximada por

$$
\left.\frac{\partial(v v)}{\partial z}\right|_{i, j+\frac{1}{2}} \frac{\hat{v}_{i, j+1} v_{i, j+1}-\hat{v}_{i, j} v_{i, j}}{\delta z}
$$

onde $\hat{v}$ é a velocidade convectiva obtida pela média dos valores vizinhos

$$
\hat{v}_{i, j+1}=\frac{v_{i, j+\frac{3}{2}}+v_{i, j+\frac{1}{2}}}{2}, \quad \hat{v}_{i, j}=\frac{v_{i, j ! \frac{1}{2}}+v_{i, j-\frac{1}{2}}}{2} .
$$

As velocidades transportadas são calculadas por meio do esquema convectivo apresentado anteriormente (CUBIST $\Lambda$ ).

Por exemplo, os valores $v_{i, j+1}$ e $v_{i, j}$ em (3.21) são obtidos como segue. Defisindo

$$
S_{i, j+1}=-\left\{\begin{array}{ll}
0, & \hat{v}_{i, j+1} \geq 0 \\
1, & \text { caso contrário }
\end{array} \quad, \quad S_{i, j}=- \begin{cases}0, & \hat{v}_{i, j} \geq 0 \\
1, & \text { caso contrário }\end{cases}\right.
$$

temos,

$$
\begin{aligned}
& \hat{\phi}_{i, j+1}=\left(1-S_{i, j 11}\right)\left(\frac{v_{i, j+\frac{1}{2}}-v_{i, j-\frac{1}{2}}}{v_{i, j+\frac{3}{2}}-v_{i, j-\frac{1}{2}}}\right) \cdot S_{i, j+1}\left(\frac{v_{i, j+\frac{3}{2}}-v_{i, j+\frac{5}{2}}}{v_{i, j+\frac{1}{2}}-v_{i, j+\frac{5}{2}}}\right) \\
& v_{i, j+1}=\left(1-S_{i, j+1}\right)\left\{\begin{array}{ll}
v_{i, j+\frac{1}{2},}, & \hat{\phi}_{i, j+1} \notin[0,1] \\
\frac{7}{4} v_{i, j ! \frac{1}{2}}-\frac{3}{4} v_{i, j-\frac{1}{2}}, & \hat{\phi}_{i, j+1} \in(0,3 / 8) \\
\frac{3}{8} y_{i, j+\frac{3}{2}}+\frac{6}{8} y_{i, j+\frac{1}{2}}-\frac{1}{8} y_{i, j-\frac{1}{2}}, & \hat{\phi}_{i, j+1} \in[3 / 8,3 / 4] \\
\frac{3}{4} v_{i, j ! \frac{3}{2}}+\frac{1}{4} v_{i, j+\frac{1}{2}}, & \hat{\phi}_{i, j ! 1} \in(3 / 4,1)
\end{array}\right\} \\
& +S_{i, j+1}\left\{\begin{array}{ll}
v_{i, j+\frac{3}{2}}, & \hat{\phi}_{i, j+1} \notin[0,1] \\
\frac{7}{4} v_{i, j+\frac{3}{2}}-\frac{3}{4} v_{i, j+\frac{5}{2}}, & \hat{\phi}_{i, j+1} \in(0,3 / 8) \\
\frac{3}{3} v_{i, j+\frac{1}{2}}+\frac{6}{8} v_{i, j+\frac{3}{2}}-\frac{1}{8} v_{i, j+\frac{5}{2}}, & \hat{\phi}_{i, j+1} \in[3 / 8,3 / 4] \\
\frac{3}{4} v_{i, j+\frac{1}{2}}+\frac{1}{4} v_{i, j+\frac{3}{2}}, & \hat{\phi}_{i, j+1} \in(3 / 4,1)
\end{array}\right\} \\
& \hat{\phi}_{i, j}=\left(1-S_{i, j}\right)\left(\frac{v_{i, j-\frac{1}{2}}-v_{i, j}-\frac{3}{2}}{v_{i, j+\frac{1}{2}}-v_{i, j-\frac{3}{2}}}\right)+S_{i, j}\left(\frac{v_{i, j}+\frac{1}{2}-v_{i, j+\frac{3}{2}}}{v_{i, j-\frac{1}{2}}-v_{i, j+\frac{3}{2}}}\right)
\end{aligned}
$$




$$
\begin{aligned}
& v_{i, j}=\left(1-S_{i, j}\right)\left\{\begin{array}{ll}
v_{i, j-\frac{1}{2}}, & \hat{\phi}_{i, j} \notin\{0,1] \\
\frac{7}{4} v_{i, j-\frac{1}{2}}-\frac{3}{4} v_{i, j-\frac{3}{2}}, & \hat{\phi}_{i, j} \in(0,3 / 8) \\
\frac{3}{8} v_{i, j+\frac{1}{2}}+\frac{6}{8} v_{i, j}-\frac{1}{2} v_{i, j}, \frac{3}{2}, & \hat{\phi}_{i, j} \in[3 / 8,3 / 4\} \\
\frac{3}{4} v_{i, j+\frac{1}{2}} \mid \frac{1}{4} v_{i, j-\frac{1}{2}}, & \hat{\phi}_{i, j} \in(3 / 4,1)
\end{array}\right\} \\
& \left\{S_{i, j}\left\{\begin{array}{ll}
v_{i, j+\frac{1}{2}}, & \hat{\phi}_{i, j} \notin|0,1| \\
\frac{7}{4} v_{i, j+\frac{1}{2}}-\frac{3}{4} v_{i, j+\frac{3}{2}}, & \hat{\phi}_{i, j} \in(0,3 / 8) \\
\frac{3}{8} v_{i, j-\frac{1}{2}}+\frac{6}{8} v_{i, j+\frac{1}{2}}-\frac{1}{8} v_{i, j+\frac{3}{2}}, & \hat{\phi}_{i, j} \in|3 / 8,3 / 4| \\
\frac{3}{4} v_{i, j} \frac{1}{2}+\frac{1}{4} v_{i, j+\frac{1}{2}}, & \hat{\phi}_{i, j} \in(3 / 4,1)
\end{array}\right\}\right.
\end{aligned}
$$

Os outros termos convectivos são aproximados de maneira análoga a este procedimento.

\subsection{Aproximação das Condições de Contorno na Super- fície Livre}

Considere as condições de contorno na superfície livre dadas pelas equações (1.64)e (1.65). Por mcio da equação (1.64) calculamos a pressão na superfície livre e por meio da equação (1.65) calcularnos a velocidade na interface. Para aplicarmos cstas condições consideramos as idóias apresentadas por Tomé et al. [12] considerando os seguintes casos:

1. Células de superfície (S) com somente uma face em contato com uma cólula vazia (E). Neste caso, assumimos que a superfície livre é vertical ou horizontal dependendo de qual face está em contato corn a célula vazia $(E)$.

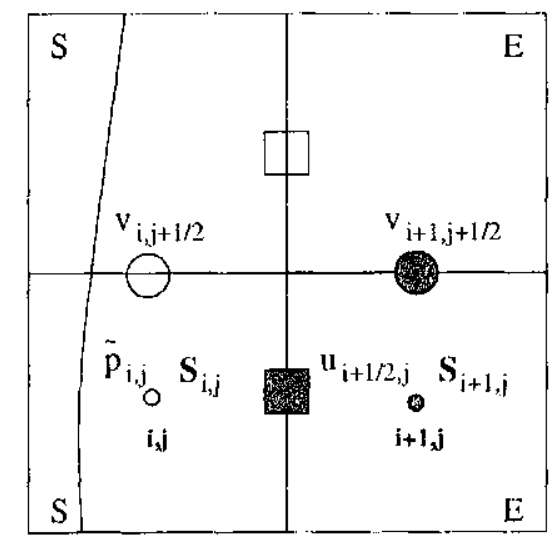

Figura 3.5: Cólula (S) com a face direita en contato com uma cólula (E). 
Consideremos o caso de uma célula de superfície com somente a face direita em contato com uma face de uma célula vazia como mostra a figura 3.5. Neste caso, tomamos $\mathbf{n}=(1,0)$ e as equações $(1.64)$ c (1.65) se reduzem a

$$
\begin{gathered}
\tilde{p}=\frac{1}{R e}\left(\frac{\lambda_{2}}{\lambda_{1}}\right)\left(2 \frac{\partial u}{\partial r}\right)+S^{r r}, \\
\frac{1}{R c}\left(\frac{\lambda_{2}}{\lambda_{1}}\right)\left(\frac{\partial u}{\partial z}, \frac{\partial v}{\partial r}\right)+S^{r z}=0 .
\end{gathered}
$$

Observamos que para o cálculo das velocidades intermediárias $\tilde{\mathbf{u}}$ usando as equações de quantidade de movimento, os valores de $\tilde{p}_{i, j}, u_{i+\frac{1}{2}, j}$ e $v_{i+1, j+\frac{1}{2}}$ são exigidos. Estes valores são obtidos pelas equações (3.22) e (3.23) c pela equação (1.20) como segue. Primeiro, discretizando a equação da continuidade no centro da célula, calculamos

$$
u_{i+\frac{1}{2}, j}=\frac{1}{r_{i+\frac{1}{2}}}\left(r_{i-\frac{1}{2}} u_{i-\frac{1}{2}, j}-r_{i} \frac{\delta r}{\delta z}\left(v_{i, j+\frac{1}{2}}-v_{i, j-\frac{1}{2}}\right)\right) .
$$

Agora, discretizando (3.23) no ponto $\left(i+\frac{1}{2}, j \mid \frac{1}{2}\right)$, temos

$$
\frac{1}{R e}\left(\frac{\lambda_{2}}{\lambda_{1}}\right)\left(\frac{u_{i+\frac{1}{2}, j+1}-u_{i+\frac{1}{2}, j}}{\delta z}+\frac{v_{i+1, j+\frac{1}{2}}-v_{i, j+\frac{1}{2}}}{\delta r}\right)+S_{i+\frac{1}{2}, j+\frac{1}{2}}^{r z}=0
$$

e isolando $v_{i \nmid 1, j \backslash \frac{1}{2}}$, vem

$$
v_{i+1, j+\frac{1}{2}}=v_{i, j+\frac{1}{2}}-\frac{\delta r}{\delta z}\left(u_{i+\frac{1}{2}, j 11}-u_{i \uparrow} \frac{1}{2}, j\right)-\operatorname{Re}\left(\frac{\lambda_{1}}{\lambda_{2}}\right) \delta r S_{i+\frac{1}{2}, j+\frac{1}{2}}^{r z},
$$

em que

$$
S_{i+\frac{1}{2}, j+\frac{1}{2}}^{r z} \frac{S_{i, j}^{r z}+S_{i \uparrow 1, j}^{r z}+S_{i, j+1}^{r z}+S_{i+1, j+1}^{r \tilde{z}}}{4}
$$

A pressão $\tilde{p}_{i, j}$ c obtida discretizando (3.22) no centro da célula $(i, j)$

$$
\tilde{p}_{i, j}=\frac{1}{\operatorname{Re}}\left[2 \frac{\lambda_{2}}{\lambda_{1}}\left(\frac{u_{i 1 \frac{1}{2}, j}-u_{i} \frac{1}{2}, j}{\delta r}\right)\right]+S_{i, j}^{r r} .
$$

As demais configurações de cólulas de superfície (S) com apenas uma face em contato com uma cólula vazia (E) são tratadas de maneira análoga.

2. Células de superfície (S) com duas faccs adjacentes em contato com células vazias (E) Nestas células assumimos que o vetor normal faz um ângulo de $45^{\circ}$ com as duas faces cm contato com célula vazia (E), ou scja, tomamos $n \quad\left( \pm \frac{\sqrt{2}}{2}, \pm \frac{\sqrt{2}}{2}\right)$.

Considerando a figura 3.6, o vetor normal toma a forma $\mathbf{n}=\left(\frac{\sqrt{2}}{2}, \frac{\sqrt{2}}{2}\right)$. $\log 0$, as equações (1.64) e (1.65) se reduzem a

$$
\tilde{p}-\frac{1}{R e}\left(\frac{\lambda_{2}}{\lambda_{1}}\right)\left(\frac{\partial u}{\partial z}+\frac{\partial u}{\partial r}+\frac{\partial v}{\partial r}+\frac{\partial v}{\partial z}\right)+\frac{1}{2}\left(S^{r r}+2 S^{r z}+S^{z z}\right)
$$




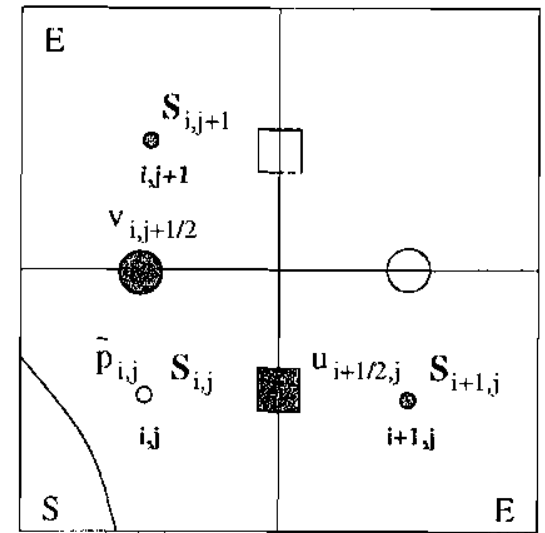

Figura 3.6: Célula (S) com duas faces em contato com cólulas (E).

$$
\frac{1}{R e} \frac{\lambda_{2}}{\lambda_{1}}\left(\frac{\partial v}{\partial z}-\frac{\partial u}{\partial r}\right)+\frac{1}{2}\left(S^{z z}-S^{r r}\right) \quad 0 .
$$

Neste caso, o cálculo do vetor velocidade e do tensor $\mathbf{S}$ requerem os valores de $\tilde{p}_{i, j}$, $u_{i+\frac{1}{2}, j}$ e $v_{i, j+\frac{1}{2}}$. Para calcular $u_{i+\frac{1}{2}, j}$ e $v_{i, j+\frac{1}{2}}$ usamos a equação da continuidade (1.20) e a condição tangencial (3.26) que discretizadas no contro da célula (S) produzen as equações

$$
\begin{gathered}
\frac{1}{r_{i}} \frac{r_{i+\frac{1}{2}} u_{i+\frac{1}{2}, j}-r_{i-\frac{1}{2}} u_{i-\frac{1}{2}, j}}{\delta r}+\frac{v_{i, j+\frac{1}{2}}-v_{i, j-\frac{1}{2}}}{\delta z} \cdots, \\
\frac{1}{\operatorname{Re}} \frac{\lambda_{2}}{\lambda_{1}}\left(\frac{v_{i, j+\frac{1}{2}}-v_{i, j} \frac{1}{2}}{\delta z} \cdots \frac{u_{i-1 \frac{1}{2}, j}-u_{i-\frac{1}{2}, j}}{\delta r}\right)+\frac{1}{2}\left(S_{i, j}^{z z} \cdots S_{i, j}^{r r}\right)=0 .
\end{gathered}
$$

As equações (3.27) e (3.28) fornecem urn sistema $2 \times 2$ para $u_{i+\frac{1}{2}, j}$ e $v_{i, j \vdash \frac{1}{2}}$. Resolvendo este sistema, obtemos

$$
\begin{aligned}
& u_{i+\frac{1}{2}, j}-\left(\frac{r_{i}+r_{i-\frac{1}{2}}}{r_{i}+r_{i+\frac{1}{2}}}\right) u_{i} \frac{1}{2}, j+\frac{R e}{2}\left(\frac{r_{i}}{r_{i}+r_{i+\frac{1}{2}}}\right) \delta r \frac{\lambda_{1}}{\lambda_{2}}\left(S_{i, j}^{z z}-S_{i, j}^{r r r}\right), \\
& v_{i, j+\frac{1}{2}}=v_{i, j}-\frac{1}{2} \frac{\delta z}{r_{i}} \frac{\delta}{\delta r}\left(r_{i+\frac{1}{2} u_{i+\frac{1}{2}, j}-r_{i_{2}} u_{i} l_{2}, j}\right) .
\end{aligned}
$$

Para o cálculo de $\tilde{p}_{i, j}$, aproximamos a equação (3.25) no centro da célula (S), obtendo

$$
\begin{aligned}
\tilde{p}_{i, j} & =\frac{1}{R e} \frac{\lambda_{2}}{\lambda_{1}}\left[\frac{u_{i+\frac{1}{2}, j}-u_{i-\frac{1}{2}, j}}{\delta r}+\frac{u_{i+\frac{1}{2}, j} \mid u_{i \frac{1}{2}, j}-u_{i \nmid \frac{1}{2}, j-1}-u_{i-\frac{1}{2}, j} 1}{\delta z}\right. \\
& \left.+\frac{v_{i, j+\frac{1}{2}}-v_{i, j} \frac{1}{2}}{\delta z}+\frac{v_{i, j \mid \frac{1}{2}}+v_{i, j \frac{1}{2}}-v_{i-1, j+\frac{1}{2}}-v_{i-1, j-\frac{1}{2}}}{\delta r}\right] \\
& +\frac{1}{2}\left(S_{i, j}^{r r}+2 S_{i, j}^{r z} \mid S_{i, j}^{z z}\right) .
\end{aligned}
$$

As demais configurações de células (S) com duas faces adjacentes em contato com células (E) são obtidos de maneira análoga a anterior. 
3. Células de superfície (S) com duas faces opostas em contato com cêlulas vazias (E) ou células de superfície (S) com três faces em contato com células vazias (E).

Para essas ć́lulas não conseguimos obter uma aproximação para o vetor normal $\mathbf{n}$. Nessas ć́lulas, o valor da velocidade numa das faces ó calculado de modo que a equação da continuidade seja satisfeita. O valor da pressão e os valores das componentes do tensor extra-tensão são considerados nulos. Se essas células aparecerem durante a execução de um dado problema, a malha deverá ser refinada para minimizar o aparccimento das mesmas.

\subsection{Cálculo das Componentes do Tensor extra-tensão em Contornos Rígidos}

Quando as equaçôes discretizadas para o cálculo do tensor extra-tensão (3.11) - (3.14) são aplicadas em nós que são adjacentes ao contorno rígido, os valores de $S^{r r}, S^{\theta \theta}, S^{z z} \mathrm{e}$ $S^{r z}$ nlas células de contorno (B) são exigidos.

Estes valores podem ser obtidos por mcio das equações derivadas na seção 1.2.1. Para isto, percorremos as células de contorno (B) c verificamos qual face está em contato com células (F) ou cólulas (S), como segue.

1. Células de contorno (B) com apcnas a face superior ou a face inferior em contato com uma célula (F) ou uma célula (S).

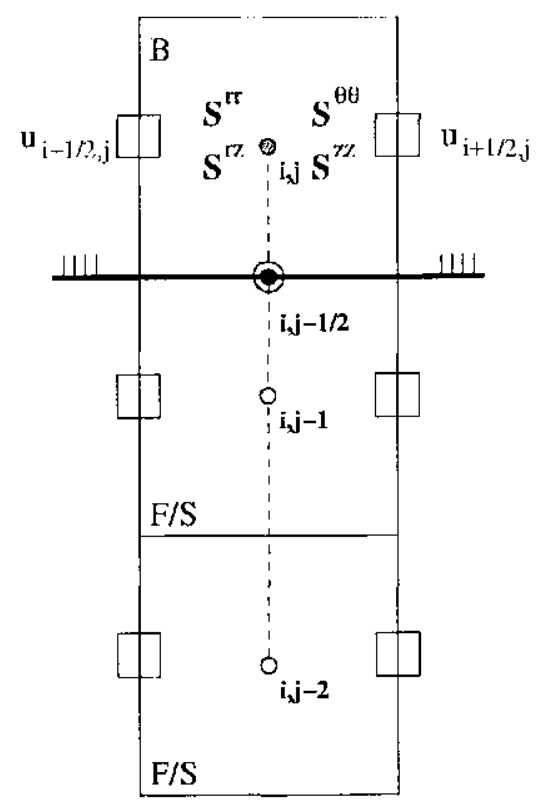

Figura 3.7: Célula (B) com a face inferior em contato com uma célula (F/S). 
Considerando a figura 3.7, assumimos que o contorno rígido é horizontal e discretizando as equações (1.38), (1.39) e (1.40), os valores das componentes do tensor extra-tensão no ponto $\left(i, j-\frac{1}{2}\right)$ são dados por

$$
\begin{aligned}
& S_{i, j-\frac{1}{2}}^{\theta \theta}{ }^{(n+1)}=0, \\
& S_{i, j \frac{1}{2}}^{z z}{ }^{(n+1)}=0 \text {, } \\
& S_{i, j \frac{1}{2}}^{r z}{ }^{(n+1)} \quad \cdots \quad e^{\frac{-\partial t}{W \epsilon}} S_{i, j}^{r z} \frac{1}{2}{ }^{(n)}+\left.\frac{1}{R e}\left(1-\frac{\lambda_{2}}{\lambda_{1}}\right) \frac{\partial u}{\partial z}\right|_{i, j-\frac{1}{2}} ^{l^{*}}\left[1-e^{\frac{-\delta t}{W e}}\right], \\
& \left.S_{i, j-\frac{1}{2}}^{r r}(n+1)=e^{\frac{\delta t}{W e}} S_{i, j-\frac{1}{2}}^{r r}+\delta t\left[\left.e^{\frac{-\delta t}{W t}} \frac{\partial u}{\partial z}\right|_{i, j} ^{t^{n}} S_{\frac{1}{2}}^{r z} S_{i, j-\frac{1}{2}}^{(n)}+\left.\frac{\partial u}{\partial z}\right|_{i, j} ^{t^{n+1}} S_{i, j-\frac{1}{2}}^{r z}{ }^{(n+1)}\right] 3.32\right)
\end{aligned}
$$

onde $\left.\frac{\partial u}{\partial z}\right|_{i, j \cdot \frac{1}{2}} ^{l^{*}}$ é calculada fazendo uma média aritmética nos tempos $t_{n}$ e $t_{n+1}$, ou seja,

$$
\left.\frac{\partial u}{\partial z}\right|_{i, j-\frac{1}{2}} ^{t^{*}}=\frac{1}{2}\left[\left.\frac{\partial u}{\partial z}\right|_{i, j \cdots \frac{1}{2}} ^{\ell^{n}},\left.\frac{\partial u}{\partial z}\right|_{i, j} ^{t^{n+1}}\right] .
$$

Estas derivadas são aproximadas da seguinte forma:

- Se a célula $(i, j-2)$ é cheia (F) ou de superfícic (S) fazemos uma aproximação de segunda ordem expandindo $u$ em série do Taylor em torno do ponto $\left(i, j-\frac{1}{2}\right)$. Neste caso, a aproximação da derivada ć feita avaliando a séric de Taylor nos pontos $(i, j-1),(i, j-2)$ obtendo (desde que $u_{i, j} \frac{1}{2}=0$ )

$$
\left.\frac{\partial u}{\partial z}\right|_{i, j-\frac{1}{2}} ^{t^{n}}-\frac{-u_{i, j}^{n}}{1}+\frac{1}{3} u_{i, j-2}^{n},\left.\quad \frac{\partial u}{\partial z}\right|_{i, j-\frac{1}{2}} ^{t^{n+1}}=\frac{-u_{i, j-1}^{n+1}+\frac{1}{3} u_{i, j}^{n+1} 2}{\delta z}{ }^{2}
$$

- Se apenas a célula $(i, j-1)$ é cheia (F) ou de superfície (S) a derivada é aproximada por diferenças avançadas, logo

$$
\left.\frac{\partial u}{\partial z}\right|_{i, j-\frac{1}{2}} ^{t^{n}}=\frac{-2 u_{i, j}^{n} 1}{\delta z},\left.\quad \frac{\partial u}{\partial z}\right|_{i, j-\frac{1}{2}} ^{l^{n+1}}=\frac{-2 u_{i, j-1}^{n+1}}{\delta z} .
$$

A vclocidade nas posições $(i, j-1)$ e $(i, j-2)$ das equações (3.33) e (3.34) são obtidas por

$$
u_{i, j-2}=\frac{u_{i+\frac{1}{2}, j-2}+u_{i-\frac{1}{2}, j-2}}{2} \text { e } u_{i, j-1} \quad \frac{u_{i+\frac{1}{2}, j-1}+u_{i-\frac{1}{2}, j} 1}{2} .
$$


Para obtermos $S_{i, j}^{r z}$ e $S_{i, j}^{r r}$ fazemos uma interpolação linear utilizando os nós $\left(i, j-\frac{1}{2}\right)$ e $(i, j-1)$, respectivamente. Para simplificar a notação, o índice $t^{n+1}$ é abandonado, uma vez que todos os termos de (3.35) - (3.38) estão no tempo $t^{(n+1)}$. Desta forma

$$
\begin{aligned}
& S_{i, j}^{\theta \theta}=-S_{i, j-1}^{\theta \theta}, \\
& S_{i, j}^{z z}=-S_{i, j-1}^{z z}, \\
& S_{i, j}^{r z}=2 S_{i, j-\frac{1}{2}}^{r z}-S_{i, j-1}^{r z}, \\
& S_{i, j}^{r r}=2 S_{i, j-\frac{1}{2}}^{r r}-S_{i, j-1}^{r r} .
\end{aligned}
$$

Para células de contorno (B) com apenas a face superior em contato com célula cheia (F) ou de superfície (S), os valores das componentes do tensor extra-tensão são obtidos de maneira análoga.

2. Células de contorno (B) com apenas a face direita ou a face esquerda em contato com uma célula (F) ou urma cólula (S).

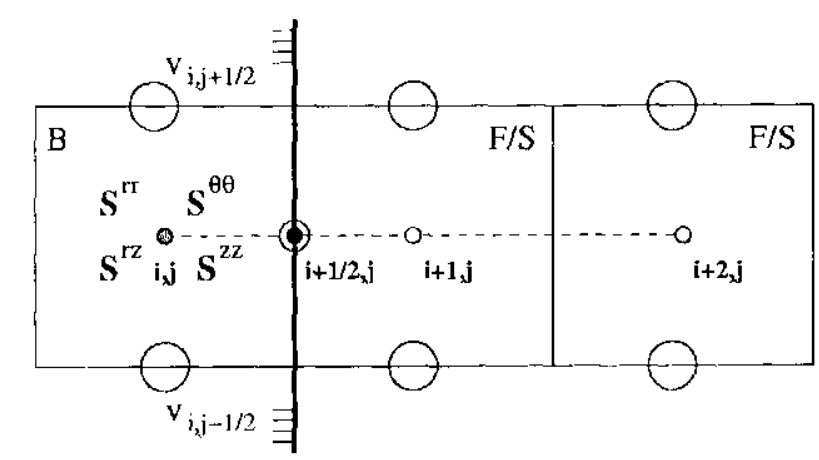

Figura 3.8: Célula (B) com a face direita em contato corn uma célula (F/S)

Considerando a figura 3.8 o cálculo das componentes do tensor extra-tensão nestas células é efetuado por meio das equações (1.45) - (1.48) obtidas na seção 1.2 .1 e são dados por

$$
\begin{aligned}
& S_{i+\frac{1}{2}, j}^{\theta \theta}(n+1)=0, \\
& S_{i+\frac{1}{2}, j}^{r r}{ }^{(n+1)}=0 \text {, } \\
& S_{i+\frac{1}{2}, j}^{r z}{ }^{(n+1)}=e^{\frac{\delta t}{W *}} S_{i+\frac{1}{2}, j}^{r z}+\left.\frac{1}{R e}\left(1-\frac{\lambda_{2}}{\lambda_{1}}\right) \frac{\partial v}{\partial r}\right|_{i-1 \frac{1}{2}, j} ^{t^{*}}\left[1-e^{\frac{-\delta t}{W e}}\right]
\end{aligned}
$$

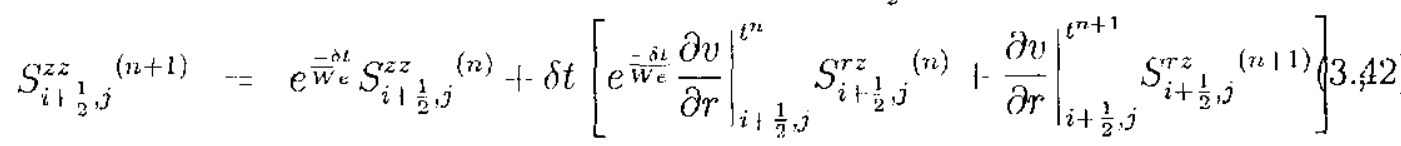


onde $\left.\frac{\partial v}{\partial r}\right|_{i+\frac{1}{2}, j} ^{t^{*}}$ é obtida fazendo uma média aritmética nos tempos $t_{n}$ e $t_{n+1}$, ou seja,

$$
\left.\frac{\partial v}{\partial r}\right|_{i, \frac{1}{2}, j} ^{t^{*}}=\frac{1}{2}\left[\left.\frac{\partial v}{\partial r}\right|_{i \rightarrow \frac{1}{2}, j} ^{t^{n}}+\left.\frac{\partial v}{\partial r}\right|_{i+1, \frac{1}{2}, j} ^{t^{n+1}}\right]
$$

Estas derivadas são aproximadas da seguinte forma:

- Se a célula $(i+2, j)$ é chcia $(F)$ on de superfícic (S) fazemos uma aproximaçäo de segunda ordem expandindo $v$ cm série de Taylor em torno do ponto $\left(i+\frac{1}{2}, j\right)$. Neste caso, a aproximaçào da derivada é feita utilizando os pontos $(i-1, j) \mathrm{c}$ (i $12, j$ ) com $v_{i+\frac{1}{2}, j}=0$

$$
\left.\frac{\partial v}{\partial r}\right|_{i \mid \frac{1}{2}, j} ^{t^{n}}-\frac{v_{i+1, j}^{n}-\frac{1}{3} v_{i+2, j}^{n}}{\delta r},\left.\quad \frac{\partial v}{\partial r}\right|_{i \mid \frac{1}{2}, j} ^{t^{n+1}}-\frac{v_{i+1, j}^{n+1}-\frac{1}{3} v_{i+2, j}^{n+1}}{\delta r}
$$

- Se apenas a célula $(i+1, j)$ ć cheia (F) ou de superfície (S) a derivada é aproximada por diferenças avançadas, logo

$$
\left.\frac{\partial v}{\partial r}\right|_{i+\frac{1}{2}, j} ^{t^{n}}=\frac{2 v_{i+1, j}^{n}}{\delta r},\left.\quad \frac{\partial v}{\partial r}\right|_{i+\frac{1}{2}, j} ^{t^{n+1}}=\frac{2 v_{i+1, j}^{n+1}}{\delta r}
$$

A velocidade nas posições $(i+1, j)$ : $(i+2, j)$ das cquações $(3.43)$ - (3.44) são obtidas por

$$
v_{i-1, j} \cdot \frac{v_{i|1, j| \frac{1}{2}+v_{i 1-1, j-1}}}{2} \text { o } v_{i+2, j}=\frac{v_{i+2, j+\frac{1}{2}} \mid v_{i+2, j-\frac{1}{2}}}{2}
$$

E por firn, para obtermos $S_{i, j}^{r z}$ c $S_{i, j}^{r r}$ fazemos urna intcrpolação lincar utilizando os nós $\left(i+\frac{1}{2}, j\right)$ c $(i \mid 1, j)$. Novamente, o indice $t^{(n+1)}$ e abandonado para simplificar a notação, logo

$$
\begin{aligned}
& S_{i, j}^{\theta \theta}=-S_{i+1, j}^{\theta \theta}, \\
& S_{i, j}^{r r}=-S_{i \cdot 1, j}^{r r}, \\
& S_{i, j}^{r z}=2 S_{i+\frac{1}{2}, j}^{r z}-S_{i+1, j}^{r z}, \\
& S_{i, j}^{z z}-2 S_{i+\frac{1}{2}, j}^{z z}-S_{i+1, j}^{z z z}
\end{aligned}
$$

Para cólulas de contorno (B) com apenas a face esquerda em contato com cólula cheia (F) ou de superfície (S), os valores das componentes do tensor extra-tensão são obtidos analogarnente. 
3. Células de contorno (B) com duas faces em contato corn uma célula (F) ou uma célula (S).

Neste caso, assumimos que o contorno rígido é paralclo aos eixos $r$ e $z$.

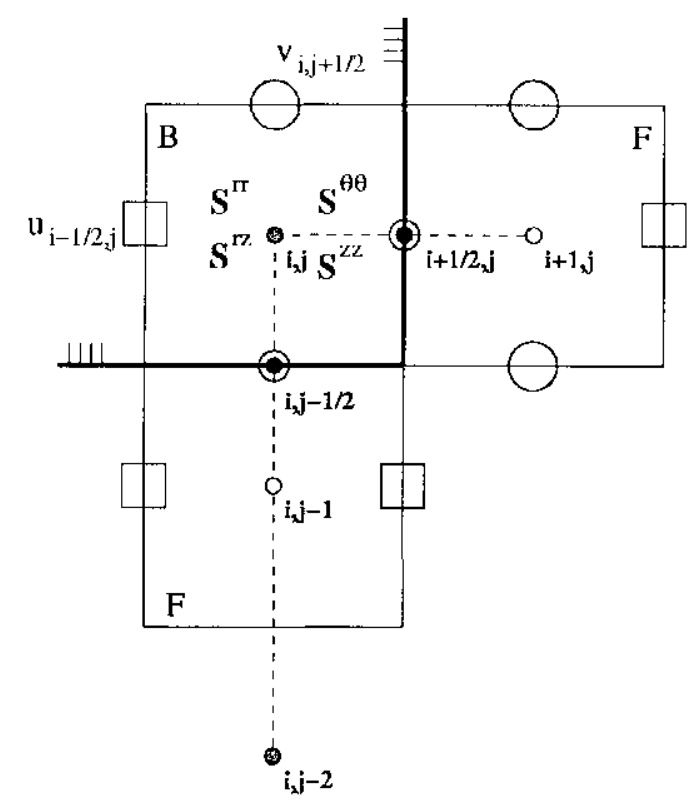

Figura 3.9: Célula (B) com a face inferior e a face direita em contato com células (F).

Nestas células, o valor das componentes do tensor extra-teısão é obtido pela média aritmética dos valores calculados nas direções $r$ e $z$. Por exemplo, considerando a figura 3.9, o cálculo das componentes do tensor extra-tensão na ć́lula (B) é dado por

$$
\begin{aligned}
& S_{i, j}^{\theta \theta}=-\frac{1}{2}\left(S_{i, j-1}^{\theta \theta}+S_{i+1, j}^{\theta \theta}\right), \\
& S_{i, j}^{r r}=S_{i, j-\frac{1}{2}}^{r r}-\frac{1}{2}\left(S_{i, j-1}^{r r}+S_{i+1, j}^{r r}\right), \\
& S_{i, j}^{z z}-S_{i+\frac{1}{2}, j}^{z z}-\frac{1}{2}\left(S_{i, j-1}^{z z}+S_{i+1, j}^{z z}\right), \\
& S_{i, j}^{r z}-S_{i, j-\frac{1}{2}}^{r z}+S_{i+\frac{1}{2}, j}^{r z}-\frac{1}{2}\left(S_{i, j}^{r z}+S_{i \uparrow 1, j}^{r z}\right) .
\end{aligned}
$$

onde $S_{i, j-\frac{1}{2}}^{r z}, S_{i, j-\frac{1}{2}}^{r r}, S_{i+\frac{1}{2}, j}^{r z}$ e $S_{i+\frac{1}{2}, j}^{z z}$ são dados pelas equações (3.31), (3.32), (3.41) e (3.42), respectivamente. A configuração de células de contorno (B) com duas faces em contato com células cheias (F) ou de superfícic (S) existe para mais sete casos e os valores das componentes do tensor extra-tensão nestes casos são obtidos de maneira análoga. 


\subsection{Cálculo das Componentes do Tensor extra-tensão no Eixo de Simetria}

De acordo com o que foi mostrado na seção 1.2 .2 , as componentes do tensor extratensão $\mathbf{S}$ no eixo de simetria são dadas pelas equações

$$
\begin{aligned}
& S^{00}(t+\delta t)=0 \\
& S^{r z}(t+\delta l)=0 \text {, } \\
& S^{r r}(t+\delta l)=e^{w^{\frac{1}{2} \delta t}} S^{r r}(l)-\frac{\delta l}{2}\left(\left.\frac{\partial v S^{r r}}{\partial z}\right|_{t, \delta t}\left|e^{\frac{-1}{w e} \delta t} \frac{\partial v S^{r r}}{\partial z}\right|_{t}\right)+\delta t\left(\left.\left.\frac{\partial u}{\partial r}\right|_{t+\delta t} S^{r r}\right|_{t+\delta t}\right. \\
& \left.+\left.\left.e^{\frac{-1}{W e} \delta t} \frac{\partial u}{\partial r}\right|_{t} S^{r r}\right|_{t}\right)+\left.\frac{2}{R e}\left(1-\frac{\lambda_{2}}{\lambda_{1}}\right) \frac{\partial u}{\partial r}\right|_{t=t^{*}}\left[1-e^{\frac{-1}{W e} \delta t}\right] \text {, } \\
& S^{z z}(t+\delta t)=e^{\frac{1}{W e} \delta t} S^{z z}(t)-\frac{\delta t}{2}\left(\left.\frac{\partial v S^{z z}}{\partial z}\right|_{t+\delta t}+\left.e^{\frac{-1}{\bar{W}} \delta t} \frac{\partial v S^{z z}}{\partial z}\right|_{t}\right)+\delta t\left(\left.\left.\frac{\partial v}{\partial z}\right|_{t+\delta t} S^{z z}\right|_{t+\delta t}\right. \\
& \left.+\left.\left.e^{\frac{-1}{\bar{w} e} \delta t} \frac{\partial v}{\partial z}\right|_{t} S^{z z}\right|_{t}\right)+\left.\frac{2}{R e}\left(1-\frac{\lambda_{2}}{\lambda_{1}}\right) \frac{\partial v}{\partial z}\right|_{t=t^{*}}\left[1-e^{\frac{1}{w e} \delta t}\right] .
\end{aligned}
$$

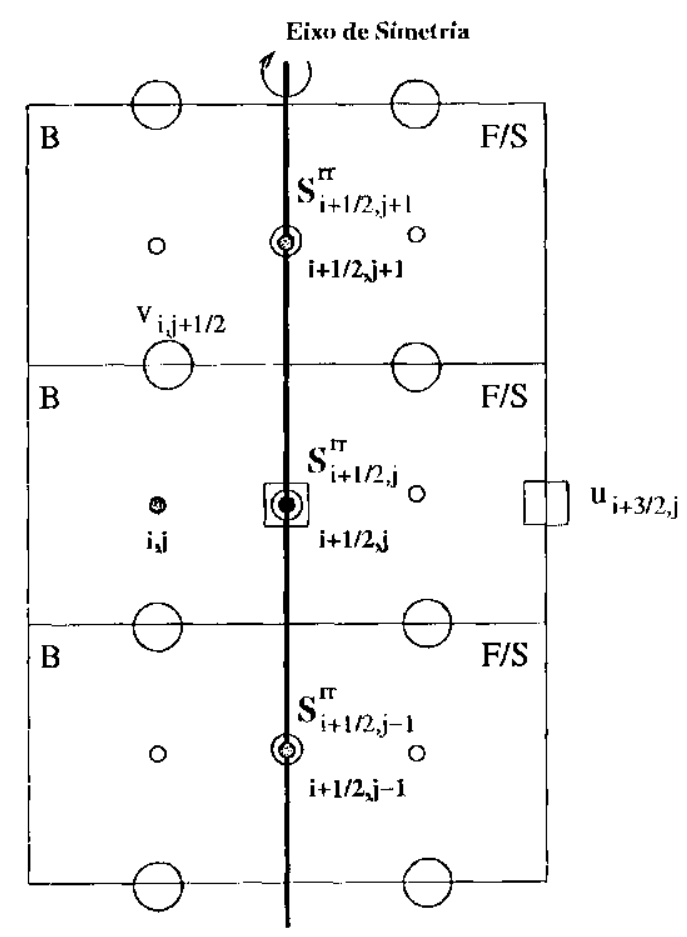

Figura 3.10: Cálculo da componente $S^{r r}$ no cixo de simetria.

Considerando a figura 3.10 , as equações (3.49) - (3.52) são aplicadas no ponto $\left(i+\frac{1}{2}, j\right)$ c discretizadas por diferenças finitas. As componentes do tensor extra-tensão são calculadas como segue. 


$$
S_{i, j}^{\theta \theta}=-S_{i+1, j}^{\theta \theta} \quad \text { e } \quad S_{i, j}^{r z}=-S_{i+1, j}^{r z} .
$$

Para obtermos os valores de $S^{r r}$ no eixo de simetria aproximamos a equação (3.51) por

$$
\begin{aligned}
\left.S_{i+\frac{1}{2}, j}^{r r}\right|^{t+\delta t} & \left.e^{\frac{-1}{W e} \delta t} S_{i+\frac{1}{2}, j}^{r r}\right|^{t}-\frac{\delta t}{2}\left\{\left[v_{i+\frac{1}{2}, j+1} S_{i+\frac{1}{2}, j 11}^{r r}-v_{i+\frac{1}{2}, j-1} S_{i+\frac{1}{2}, j-1}^{r r}\right]^{t+\delta t}\right. \\
+ & \left.e^{\frac{1}{W \epsilon} \delta t}\left[v_{i+\frac{1}{2}, j+1} S_{i+\frac{1}{2}, j+1}^{r r}-v_{i+\frac{1}{2}, j-1} S_{i+\frac{1}{2}, j-1}^{r r}\right]^{t}\right\}+\delta t\left\{\left[\left.\frac{\partial u}{\partial r}\right|_{i+\frac{1}{2}, j} S_{i+\frac{1}{2}, j}^{r r}\right]^{\ell+\delta t}\right. \\
& \left.+e^{\frac{-1}{W t} \delta t}\left[\frac{\partial u t}{\partial r} S_{i+\frac{1}{2}, j}^{r r}\right]^{\iota}\right\}+\left.\frac{2}{R e}\left(1-\frac{\lambda_{2}}{\lambda_{1}}\right) \frac{\partial u}{\partial r}\right|_{i+\frac{1}{2}, j} ^{t=\iota^{*}}\left[1-e^{\frac{-1}{W e} \delta t}\right]
\end{aligned}
$$

As derivadas $\frac{\partial u}{\partial r}$ da equação acima são aproximadas por diferenças avançadas

$$
\left.\frac{\partial u}{\partial r}\right|_{i+\frac{1}{2}, j}-\frac{u_{i+\frac{3}{2}, j}-u_{i+\frac{1}{2}, j}}{\delta r}
$$

sendo $u_{i+\frac{1}{2}, j}=0$ (pela condição de não-escorregamento).

A equação (3.54) pode ser escrita como

$$
\begin{aligned}
& {\left[v_{i+\frac{1}{2}, j} S_{1} S_{i+\frac{1}{2}, j+1}^{r r}+\left(1-\left.\delta t \frac{\partial u}{\partial r}\right|_{i+\frac{1}{2}, j}\right) S_{i+\frac{1}{2}, j}^{r r}+v_{i+\frac{1}{2}, j+1} S_{i+\frac{1}{2}, j \mid 1 !}^{r r}\right]^{\ell \mid \delta \iota}=} \\
& \left\{e^{\frac{1}{W_{\epsilon}} \delta t} S_{i+\frac{1}{2}, j}^{r r}+\frac{\delta l}{2} e^{\frac{-1}{W_{k}} \delta t}\left[v_{i+\frac{1}{2}, j+1} S_{i+\frac{1}{2}, j+1}^{r r}-v_{i+\frac{1}{2}, j-1} S_{i+\frac{1}{2}, j-1}^{r r}\right]+\delta t e^{\frac{-1}{W_{e}} \delta l} \frac{\partial u}{\partial r} S_{i+\frac{1}{2}, j}^{r r}\right\}^{t} \\
& +\left.\frac{2}{R e}\left(1-\frac{\lambda_{2}}{\lambda_{1}}\right) \frac{\partial u}{\partial r}\right|_{i+\frac{1}{2}, j} ^{t=t^{*}}\left[1-e^{\frac{-1}{\bar{w}_{e}} \delta l}\right]
\end{aligned}
$$

Para obtermos as velocidades $v_{i+\frac{1}{2}, j-1}$ e $v_{i+\frac{1}{2}, j+1}$ fazemos a média das velocidades mais próximas, isto é,

$v_{i+\frac{1}{2}, j+1} \quad \frac{v_{i, j+\frac{3}{2}}+v_{i+1, j+\frac{3}{2}}+v_{i, j+\frac{1}{2}}+v_{i+1, j+\frac{1}{2}}}{4}, \quad v_{i+\frac{1}{2}, j-1}=\frac{v_{i, j-\frac{1}{2}}+v_{i+1, j-\frac{1}{2}}+v_{i, j-\frac{3}{2}}+v_{i+1, j-\frac{3}{2}}}{4}$

A equação (3.55) resulta num sistema linear tridiagonal para o cálculo da componente $S_{i+\frac{1}{2}, j}^{r r}(t+\delta t)$ no eixo de simetria. Para resolvermos este sisterna utilizaremos o método de 
Eliminação de Gauss com pivotamento parcial. De forma análoga, a cquação (3.52) fornece

$$
\begin{aligned}
& {\left[v_{i+\frac{1}{2}, j} S_{1} S_{i+\frac{1}{2}, j-1}^{z z}+\left(1-\left.\delta t \frac{\partial v}{\partial z}\right|_{i+\frac{1}{2}, j}\right) S_{i+\frac{1}{2}, j}^{z z}+v_{i+\frac{1}{2}, j+1} S_{i+\frac{1}{2}, j+1}^{z z}\right]^{t+\delta t}=} \\
& \left\{e^{-\frac{1}{W_{e}} \delta t} S_{i+\frac{1}{2}, j}^{z z}+\frac{\delta l}{2} e^{\frac{1}{W_{e}} \delta t}\left[v_{i+\frac{1}{2}, j+1} S_{i+\frac{1}{2}, j+1}^{z z}-v_{i+\frac{1}{2}, j-1} S_{i+\frac{1}{2}, j-1}^{z z}\right]+\delta l e^{-1} \delta e^{-1 \delta} \frac{\partial u}{\partial r} S_{i+\frac{1}{2}, j}^{z z}\right\}^{l} \\
& +\left.\frac{2}{R e}\left(1-\frac{\lambda_{2}}{\lambda_{1}}\right) \frac{\partial v}{\partial z}\right|_{i+\frac{1}{2}, j} ^{t-t^{*}}\left[1-e^{\frac{-1}{W c} \delta t}\right] .
\end{aligned}
$$

Essa equação também é resolvida pelo método Eliminação de Gauss com pivotamento parcial para o cálculo de $S_{i+\frac{1}{2}, j}^{z z}(t+\delta t)$.

\subsection{Cálculo das Componentes do Tensor extra-tensão em 'Inflows' e 'Outflows'}

Considerando as condições da seção 1.2 .3 , temos que nos 'inflows' $\mathbf{S}=\mathbf{0}$ e nos 'outflows' a condição homogênea de Neumann ó imposta.

(1) Células 'Inflow' (I) situadas na face inferior da célula injetora.

Para estas células o inflow coincide com a face injetora conforme a figura 3.11 .

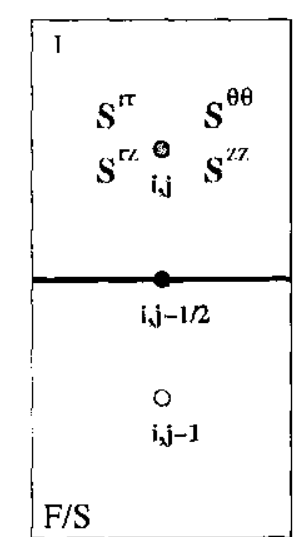

Figura 3.11: Célula 'Inflow' (I) corn a face inferior em contato com uma célula (F) ou uma célula (S). 
Neste caso, os valores das componentes do tensor extra-tensão são dados pela equação $(1.60)$

$$
\begin{aligned}
& S_{i, j}^{\theta \theta}=-S_{i, j-1}^{\theta \theta}, \\
& S_{i, j}^{r r}=-S_{i, j \quad 1}^{r r} \quad \\
& S_{i, j}^{r z}=-S_{i, j-1}^{r z}, \\
& S_{i, j}^{z z}=-S_{i, j-1}^{z z}
\end{aligned}
$$

Para as outras configurações de células 'Inflow' (I) em contato com célula (F) ou célula (S), as cormponentes do tensor extra-tensão são calculados de maneira análoga.

\section{(2) Células 'Outflow' (O) situadas na face superior da célula ejetora.}

Considerando a figura 3.12 , temos que o contorno coincide com a aresta horizontal.

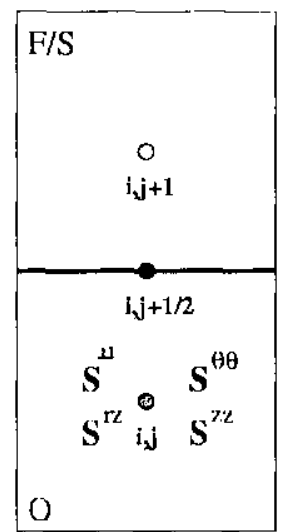

Figura 3.12: Cólula 'Outflow' $(\mathrm{O})$ com a face superior em contato com uma célula $(\mathrm{F})$ ou uma célula (S).

Neste caso, os valores das componentes do tensor extra-tensão são dados pela equação $(1.61)$

$$
\begin{aligned}
& S_{i, j}^{\theta \theta}-S_{i, j+1}^{\theta 0}, \\
& S_{i, j}^{r r}=S_{i, j+1}^{r r}, \\
& S_{i, j}^{r z}=S_{i, j+1}^{r z}, \\
& S_{i, j}^{z z}=S_{i, j-1}^{z z} .
\end{aligned}
$$

Para as outras configurações de cćlula 'Outflow'(O) em contato com cćlula (F) ou célula (S), as componentes do tensor extra-tensão são calculados de maneira análoga. 


\section{Capítulo 4}

\section{Resultados Numéricos e Validação do Método Numérico}

As equações de diferenças finitas desenvolvidas no capítulo anterior foram implementadas no sistcrna de simulação Freeflow- $\Lambda$ XI para que o mesmo possa simular escoamentos viscoclásticos governados pela cquação constitutiva Oldroyd-B. O sistema Freeflow-AXI foi aplicado para simular a escoamento ern um tubo e também para simular os seguintes problemas: inchamento do extrudado, gota incidindo contra uma superfície rígida e 'splashing drop'.

\subsection{Validação do Método Numérico}

Para validar o método numérico apresentado neste trabalho simulamos o problema do escoamento totalmente desenvolvido em um tubo c comparamos os resultados numéricos com a solução analítica para este problema, como segue.

Consideramos um tubo de raio $L$ e de comprimento $5 L$ (ver figura 4.1). Na cntrada do tubo foram impostas as condições para escoamento totalmente desenvolvido, cujas expressões para a velocidade e para as componentes do tensor extra-tensão são dadas por

$$
\begin{gathered}
v(r)=\frac{2 U_{0}\left(L^{2}-r^{2}\right)}{L^{2}} ; \quad u=0 ; \\
S^{r z}=\frac{1}{R c}\left(1-\frac{\lambda_{2}}{\lambda_{1}}\right)\left(\frac{\partial v}{\partial r}\right) ; \quad S^{z z}=2 W e S^{r z}\left(\begin{array}{c}
\partial v \\
\frac{v}{\partial r}
\end{array}\right) ; \quad S^{r r}=0 ; \quad S^{\theta \theta}=0 .
\end{gathered}
$$

Na saída do tubo são aplicadas as condições de contorno apresentadas na seção 3.11 . Na parede do tubo, impomos a condição de naão-escorregamento para a velocidade e as expressões para as componentes do tensor extra-tensão apresentadas na seção 3.9. No eixo 


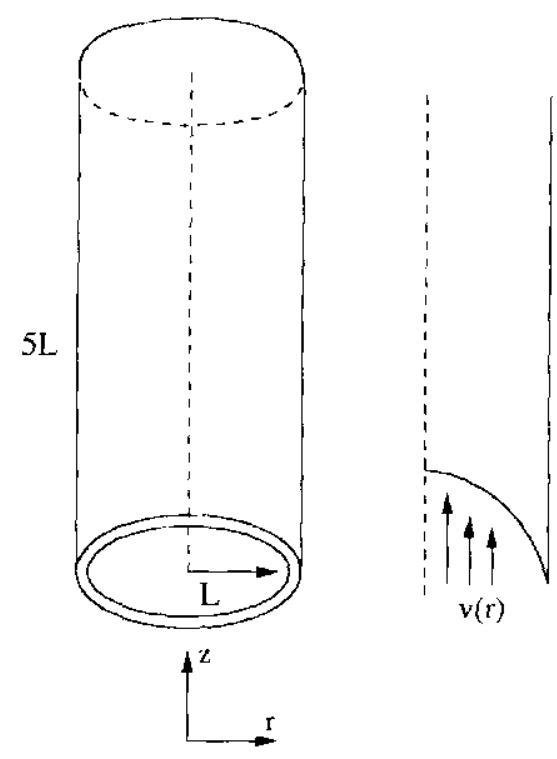

Figura 4.1: Domínio computacional para a simulaçăo do escoamento em um tubo.

de simetria impomos a condição de cscorregamento para a velocidade e para as componentes do tensor extra-tensão utilizamos as expressões obtidas na seção 3.10 .

Para simular este problema utilizamos os seguintes dados de entrada

- domínio computacional: $5.0 \mathrm{~m} \times 1.0 \mathrm{~m}$;

- velocidade de escala do fluido na entrada do tubo: $U_{0}=1.0 \mathrm{~ms}^{-1}$;

- viscosidade cincmática: $\nu=1.0 \mathrm{~m}^{2} \mathrm{~s}^{-1}$;

- raio do tubo: $L=1.0 \mathrm{~m}$;

- $\lambda_{1}=0.5, \lambda_{2}=0.25$. Logo, Re $\ldots U_{0} L / \nu-1.0$ e $W e \ldots \lambda_{1}\left(U_{0} / L\right)=0.5$.

A simulação começou no tempo $t=0 s$ com o tubo completamente vazio e o fluido foi injetado na entrada do tubo até o mesmo ficar totalmente cheio e o escoamento atingir o estado estacionário $(l=50 \mathrm{~s})$. Após o estado estacionário ser atingido, os valores da velocidade e das componentes do tensor extra-tensão em qualquer seção transversal do tubo devem ser os mesmos que os impostos na entrada do tubo.

Para verificarmos este fato e compararmos os resultados numćricos com a solução analítica dada pelas equações (4.1) e (4.2), a simulação foi realizada utilizando três malhas. 

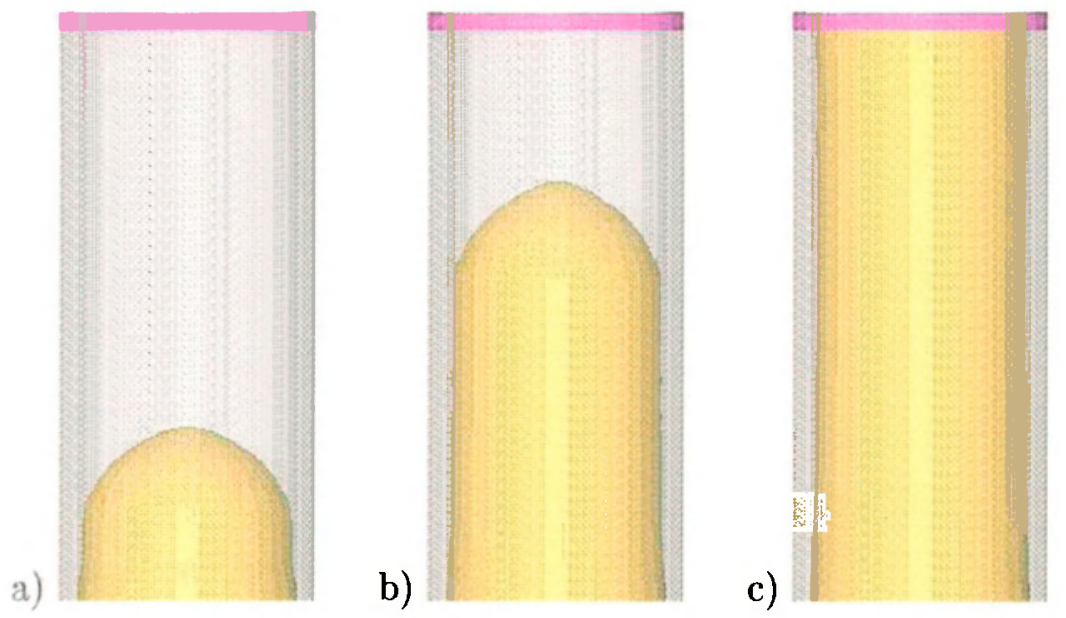

Figura 4.2: Simulação numérica do escoamento no tubo. Visualização tridimensional frontal nos tempos: a) $t=1.0$, b) $t=3.0$ e c) $t=50.0$.

- Malha 1 (grossa): $25 \times 5$ células; $\delta r=\delta z=0.2$.

- Malha 2 (intermediária): $50 \times 10$ células; $\delta r=\delta z=0.1$.

- Malha 3 (fina): $100 \times 20$ células; $\delta r=\delta z=0.05$.

A figura 4.3 mostra os resultados obtidos numericamente comparados com a solução analítica para a componente de velocidade $v$ e para as componentes do tensor extra-tensão $S^{r z}$ e $S^{z z}$ em $t=50 s$ para $y=2.5 m$ (meio do tubo).

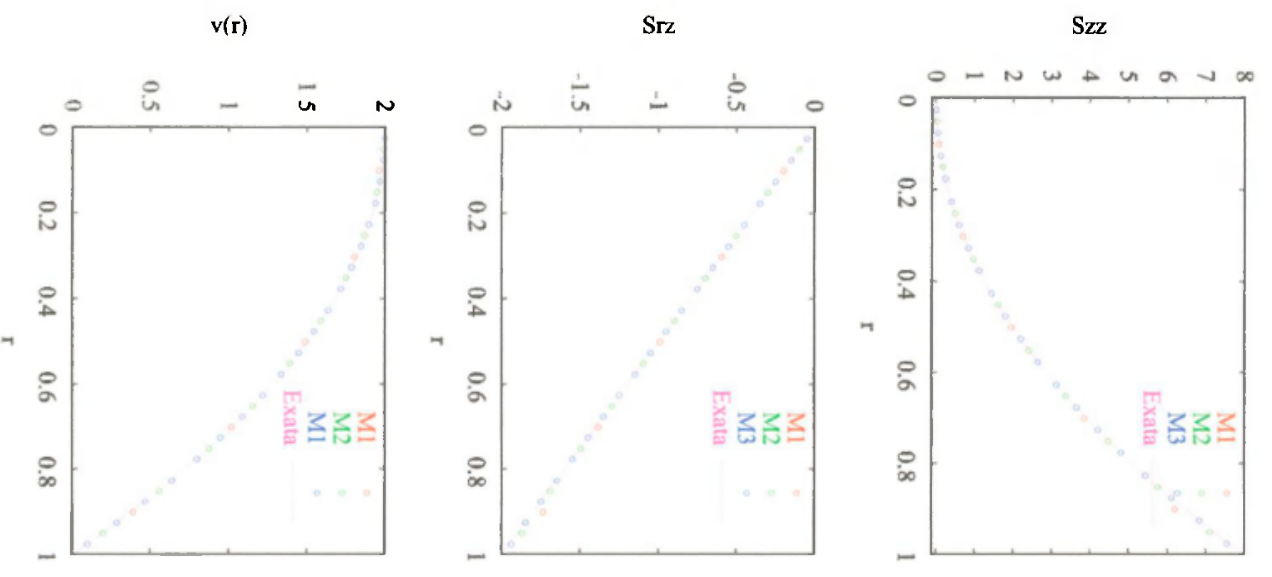

Figura 4.3: Solução numérica \& Solução analítica.

Por meio da figura 4.3 podemos observar que há uma ótima concordância entre a solução analítica e a solução numérica obtida nas três malhas e que ao refinarmos a malha a solução numérica aproxima-se mais da solução analítica. Estes resultados também validam o tratamento utilizado para o cálculo das componentes do tensor não-newtoniano 
$\left(S^{r r}, S^{r z}, S^{\theta \theta}, S^{z z}\right)$ na parede do tubo e no eixo de simetria, conforme apresentado nas seções 3.9 e 3.10 , respectivamente.

Para verificarmos a convergência do método numérico apresentado calculamos o erro relativo entre a solução analítica e a solução numérica na norma $l_{2}$ usando as três malhas.

$$
E(\text { SolNum })=\frac{\sum(\text { SolEx }- \text { SolNum })^{2}}{\sum(\text { SolEx })^{2}} .
$$

Os resultados obtidos são mostrados na tabcla a seguir onde podemos observar que os erros decrescem a medida que a malha é refinada.

\begin{tabular}{|c|c|c|c|}
\hline & Malha1 & Malha2 & Malha3 \\
\hline$E(v(r))$ & $7.810 E-5$ & $5.520 E-6$ & $3.554 E-7$ \\
\hline$E\left(S^{r z}\right)$ & $3.365 E-4$ & $2.227 E-5$ & $1.420 E-6$ \\
\hline$E\left(S^{z z}\right)$ & $1.349 E-3$ & $9.111 E-5$ & $5.853 E-6$ \\
\hline
\end{tabular}




\subsection{Resultados Numéricos}

Para demonstrarmos que a técnica numérica empregada neste trabalho pode simular escoamentos viscoelásticos com superfícies livres em movimento, são apresentados três exemplos muméricos onde podemos notar o comportamento viscoelástico. Fm cada um destes exemplos, o fator $\lambda_{2} / \lambda_{1}$ assumiu o valor unitário para representar um fluido newtoniano.

Foram obtidos resultados numéricos para os problemas do inchamento do extrudado, impacto de uma gota incidindo contra uma superfície rígida e o 'splashing drop'.

\subsubsection{Simulação Numérica do Inchamento do Extrudado}

Considere o escoamento de um fluido dentro de um tubo que ao ser expelido para a atmosfera exibe o fenômeno conhecido como inchamento do extrudado.

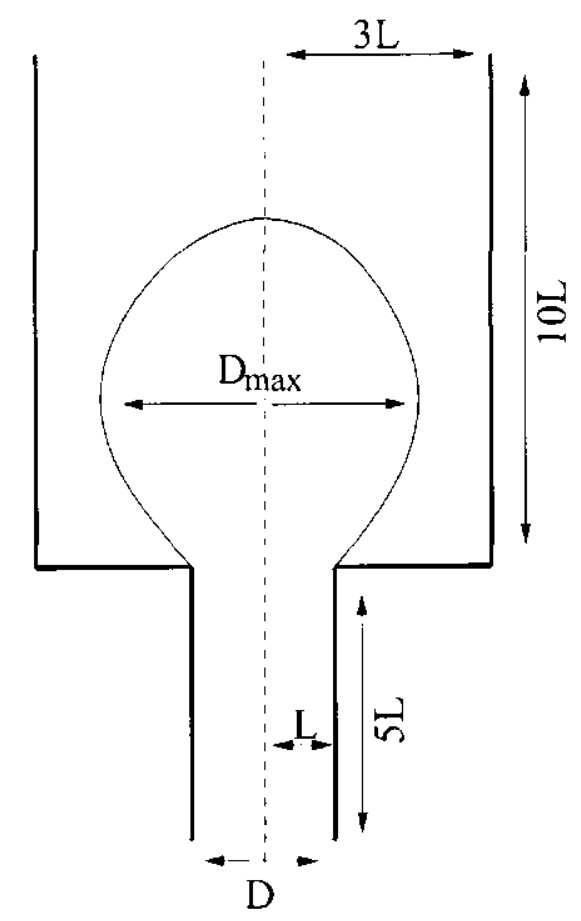

Figura 4.4: Domínio computacional para a simulação do inchamento do extrudado.

Este fenômeno é caracterizado pelo aumento do diâmetro do extrudado em relação ao diâmetro interno do tubo e vom sendo estudado por vários pesquisadores como, Tanner [20], Ryan \& Dutta [21], Crochet \& Kcunings [22] e Tomé et al. [12]. O inchamento do extrudado ć calculado pela relação entre a dilatação sofrida pelo fluido após emergir na atmosfera e o diâmetro do tudo que o fluido ocupava. Fsssa dilatação é quantificada pelo 
parâmetro:

$$
S_{r}=\frac{D_{m u: x}}{D},
$$

onde $D_{\text {mux }}$ e $D$ são dados conforme a figura 4.4 .

Para simularmos este problema, aplicamos a condição de não-escorregamento na parede do tubo enquanto que as condições de simetria apresentadas na seção 3.10 são aplicadas no cixo de simetria. Os valores da velocidade e das componentes do tensor extra-tensão na entrada do tubo são dados pelas equações (4.1) e (4.2). Na superfície livre do tubo, impomos as condições de contorno dadas pelas equações (1.64) e (1.65).

Foram realizadas três simulações com diferentes valores de Weissenberg nas quais empregamos o número de Weissenberg efetivo. O número de Weissenberg efetivo para o modelo Oldroyd-B é definido como (Yoo e Na, [19])

$$
W e_{\text {effect }}=\left(1-\frac{\lambda_{2}}{\lambda_{1}}\right) W c .
$$

Nestas simulações utilizamos os seguintes dados de entrada:

- Raio do tubo: $L=0.01 \mathrm{~m}$

- Distância do tubo ao ejetor: $10 /=0.1 \mathrm{~m}$;

- Espaçamento da malha: $\delta r=\delta z=0.001 \mathrm{~m}(10 \times 150$ células na malha $)$;

- Velocidade de escala do fluido no injetor: $U=1.00 \mathrm{~m}^{\prime-1}$;

- aceleração gravitacional: $g_{r}=g_{z}=0$;

- Parâmetros de escala: $L=0.01, U=1, \nu_{0}=0.01$. Portanto, Re $=1$ e $W e=1$.

- Definição do fluido: $\lambda_{1}=0.01$.

- Simulação 1: $\lambda_{2}=0.008 \Rightarrow W e_{\text {effect }}=0.2$

- Simulação 2: $\lambda_{2}=0.005 \Rightarrow W e_{\text {effect }}=0.5$

- Simulação 3: $\lambda_{2}=0.002 \Longrightarrow W e_{\text {effect }}=0.8$

A figura 4.5 mostra o jato escoando através de um tubo até ser expelido para a atmosfera crn diferentes tempos. No tempo $t=0.04 \mathrm{~s}$ os jatos mantém-se dentro do tubo não sendo possível notar qualquer diferença entre os três casos. A partir do momento em que o fluido deixa o tubo podemos notar as diferenças no tamanho do inchamento do extrudado para os três casos. A figura 4.6 mostra a visualização tridimensional frontal do escoamento $\mathrm{cm}$ diferentes tempos.

A partir do tempo $l=0.12 s$, podemos notar o aumento do inchamento do extrudado, desde o $W e_{\text {effect }}=0.2$ até o maior número de Weissenberg efetivo apresentado, We effect $=$ 
0.8. Podemos perceber que, no tempo $t=0.24 \mathrm{~s}$, o primeiro jato, em que $W e_{\text {effect }}=0.2$, não apresenta grande inchamento e já atinge o ejetor cnquanto que os outros dois casos ainda encontram-se em desenvolvimento. $O$ último jato a atingir o ejetor é o que apresenta maior inchamento em que $W e_{\text {effect }}=0.8$.

A razão de inchamento, $S_{r}=\frac{D_{\text {max }}}{D}$, varia aproximadamente de $65 \%$, no caso de $W c_{\text {effect }}=0.2$ até $98 \%$, no caso de $W e_{\text {effect }}=0.8$. Fisses resultados são coerentes com os encontrados na literatura e serão explicados mais detalhadamente a seguir. 


$$
t=0.04 s
$$
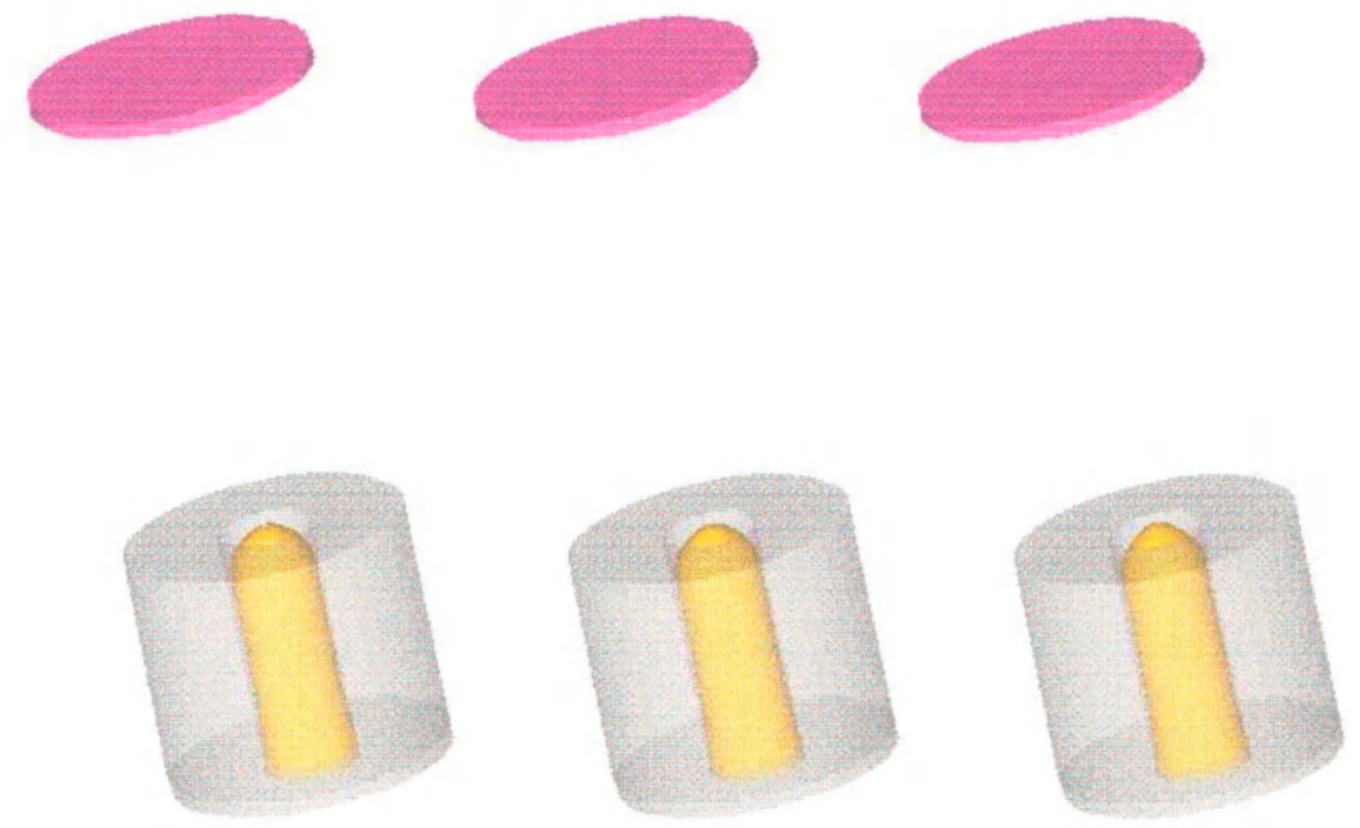

$$
t=0.06 s
$$
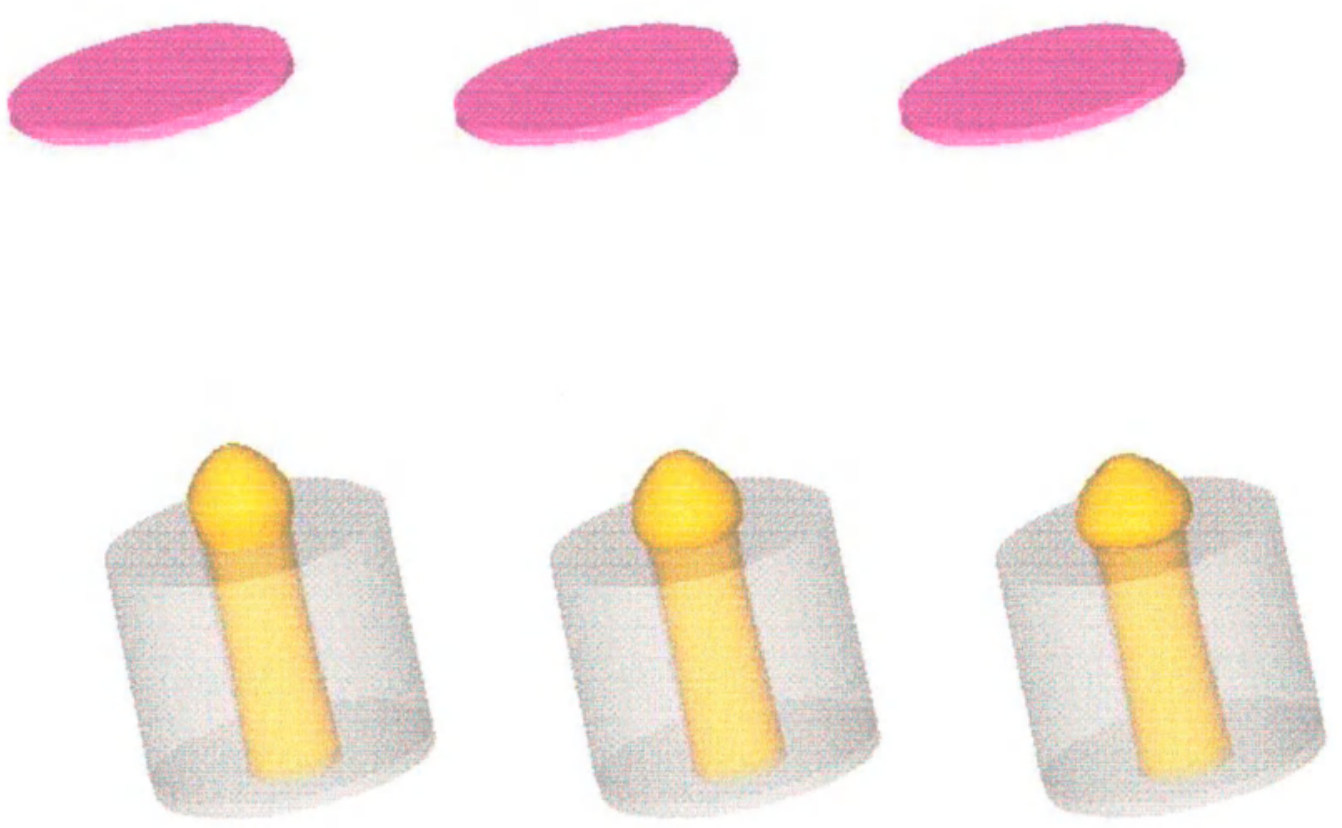

Figura 4.5: Simulação do inchamento do extrudado. We effect $=0.2$ (a esquerda), $W e_{\text {effect }}=0.5$ (no meio) e $W^{r} e_{\text {effect }}=0.8$ (a direita). Visualização tridimensional do fluido em diferentes tempos. 
$t=0.12 s$
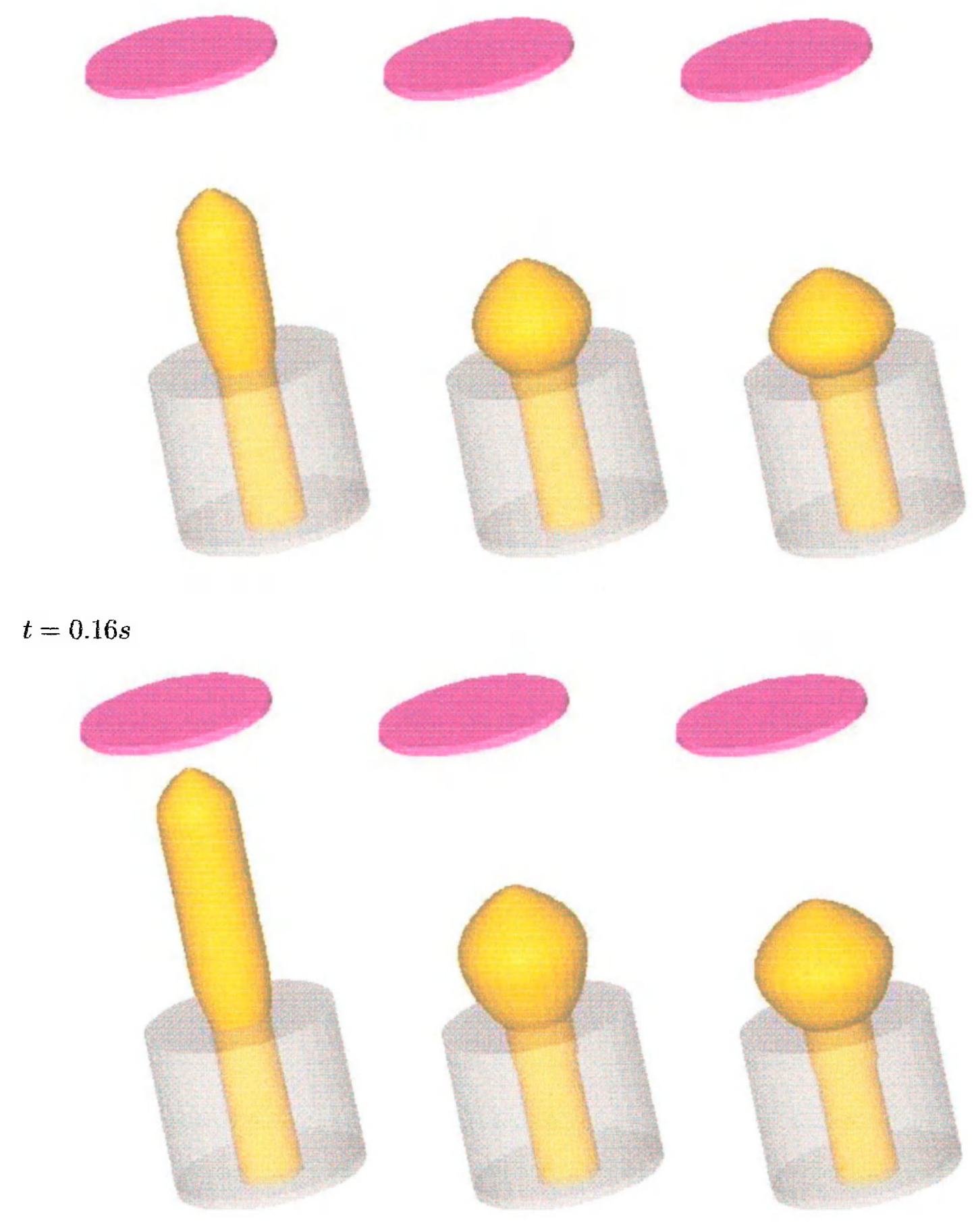

Figura 4.5: Continuação.

45 
$t=0.18 s$
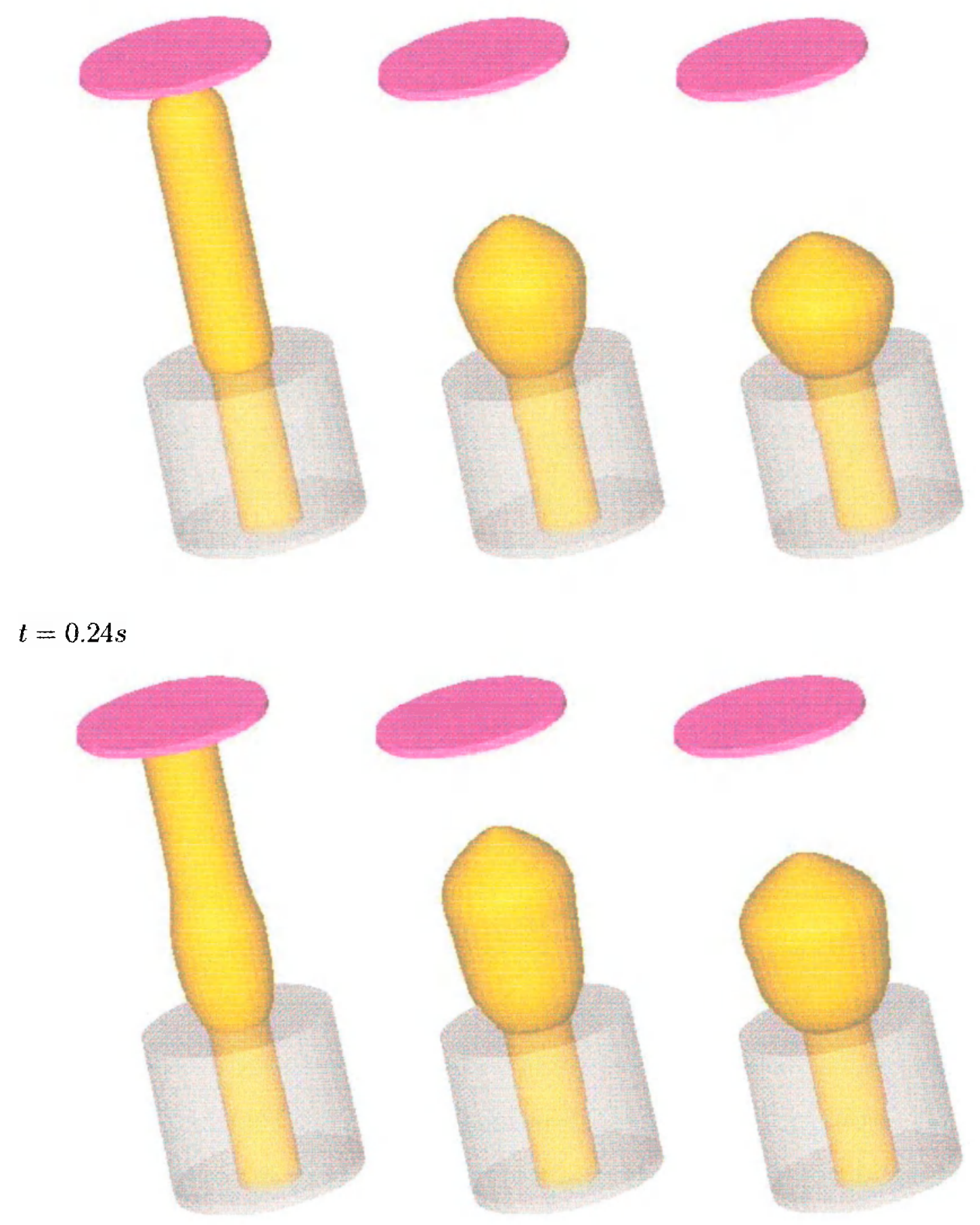

Figura 4.5: Continuação.

46 


$$
\text { If }
$$


$t=1.44 \mathrm{~s}$
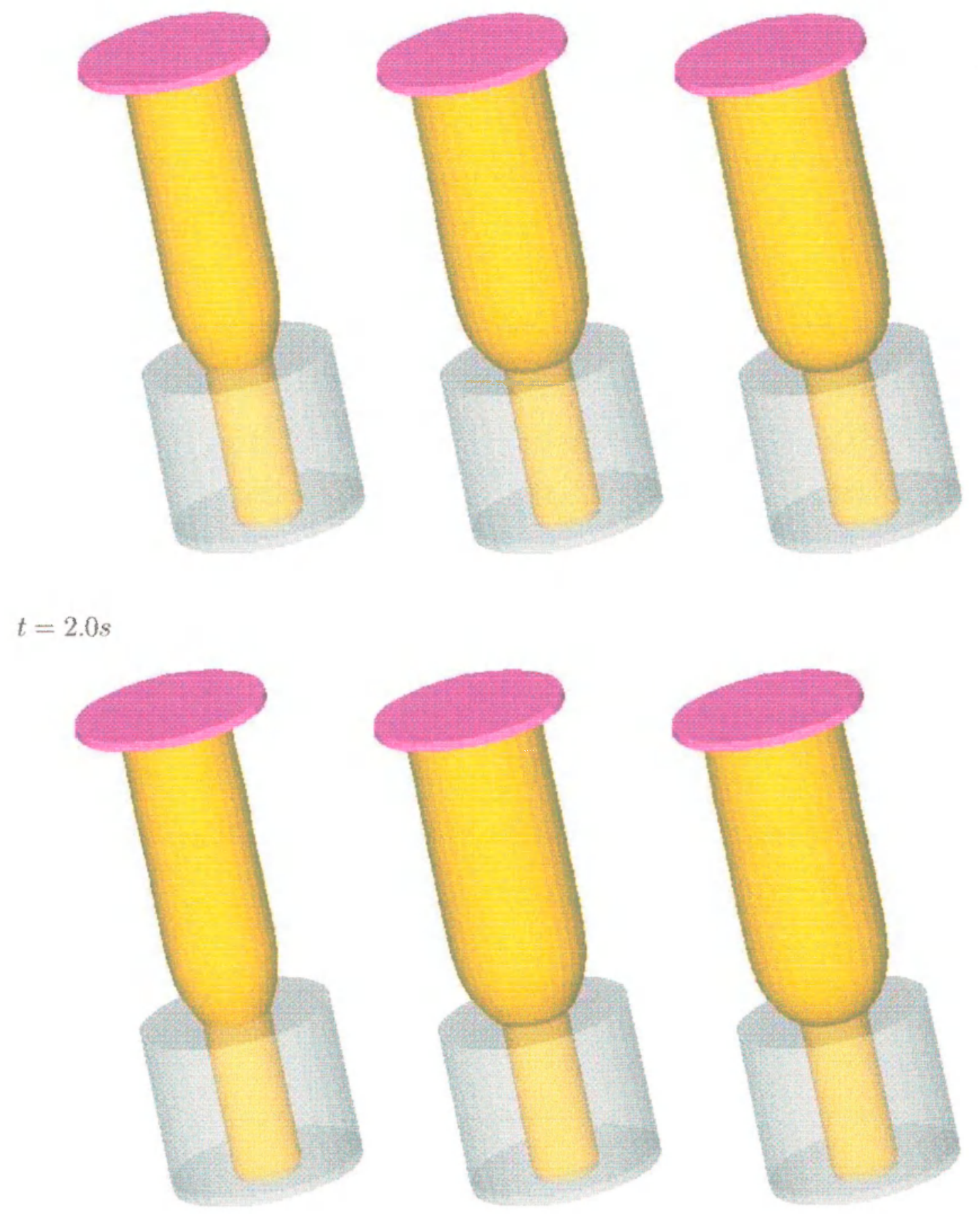

Figura 4.5: Continuação.

48 
$t=0.04 s$
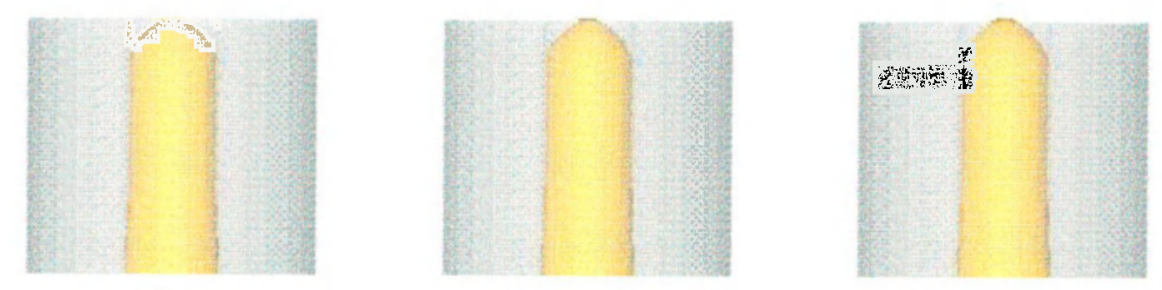

$t=0.06 s$
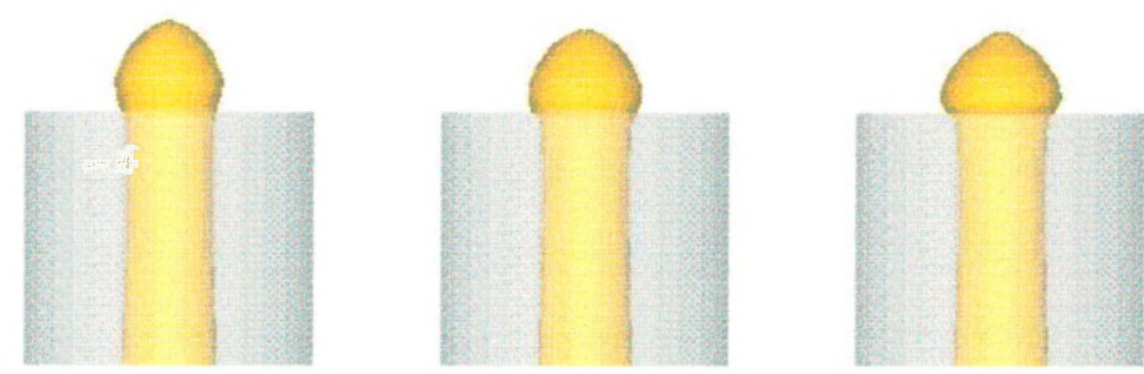

Figura 4.6: Simulação do inchamento do extrudado. $W e_{\text {effect }}=0.2($ a esquerda $), W e_{\text {effect }}=$ 0.5 (no meio) e We effect $=0.8$ (a direita). Visualização tridimensional frontal do fluido $\mathrm{cm}$ diferentes tempos. 
$t=0.12 s$
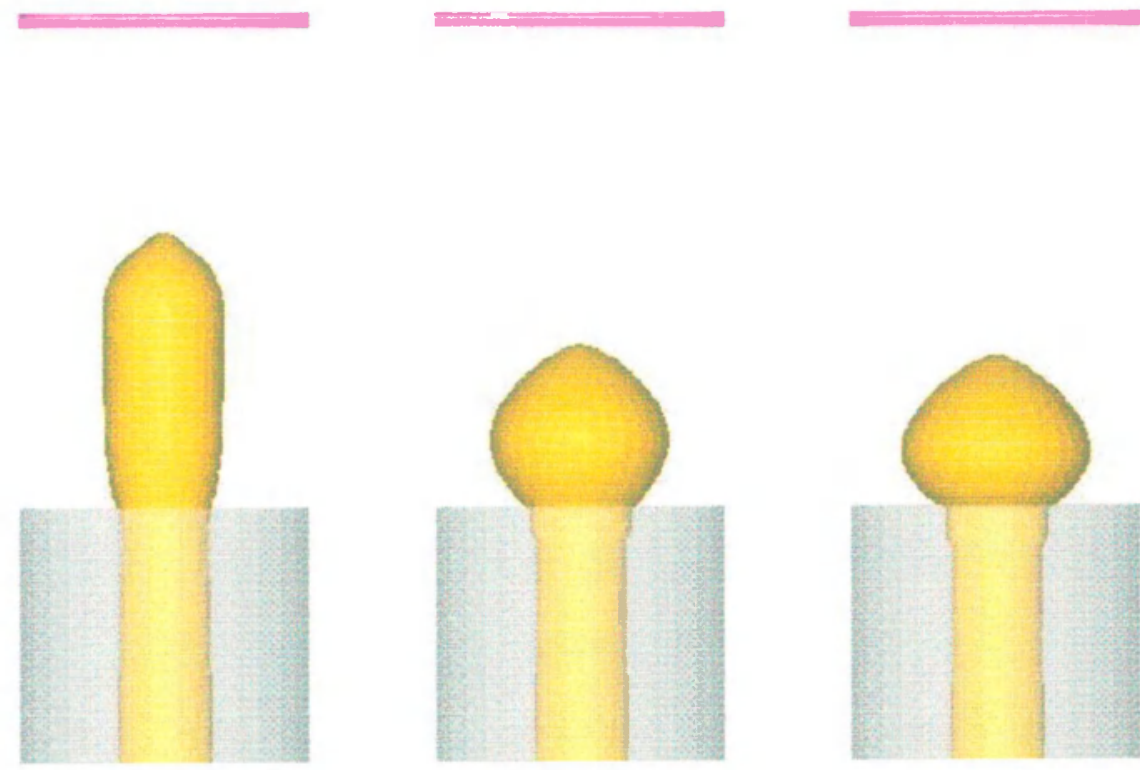

$t=0.16 \mathrm{~s}$
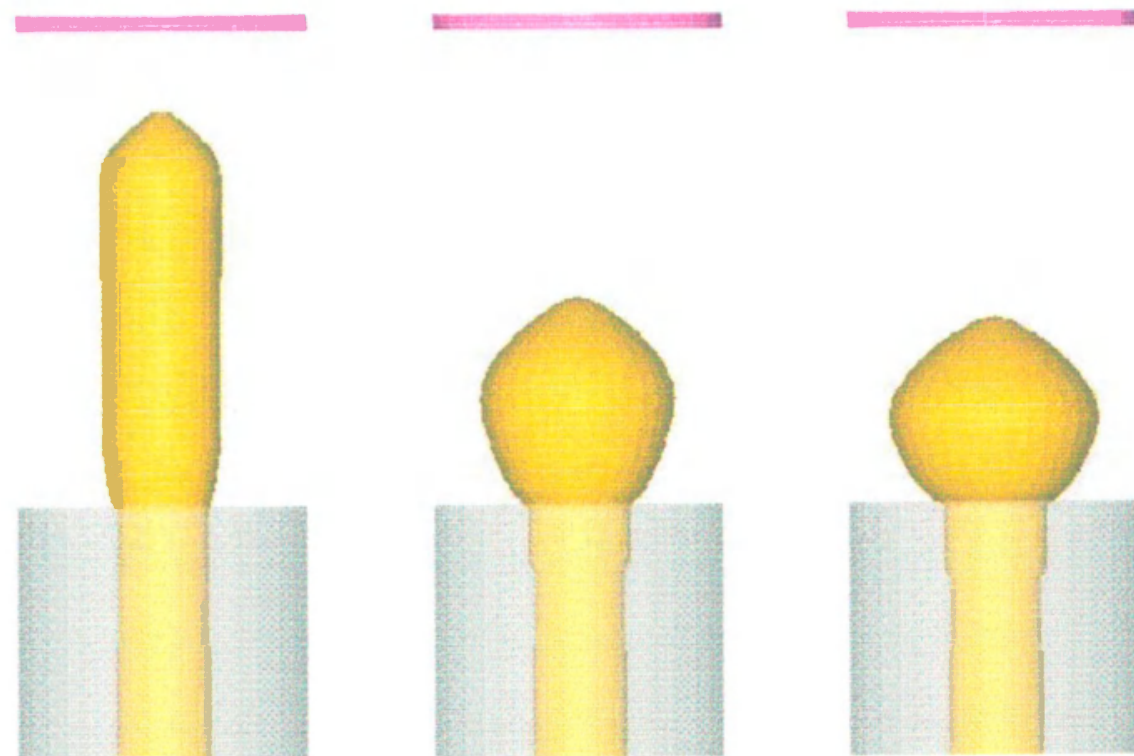

Figura 4.6: Continuação. 
$t=0.18 \mathrm{~s}$
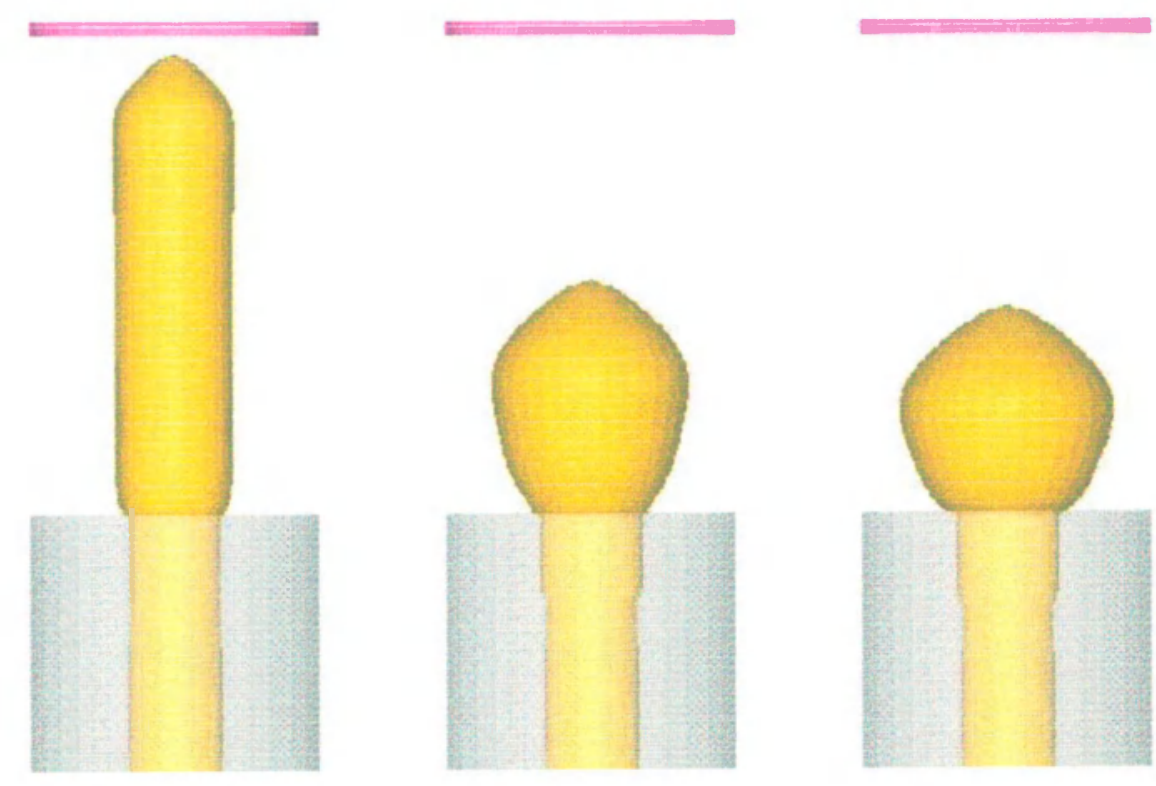

$t=0.24 \mathrm{~s}$
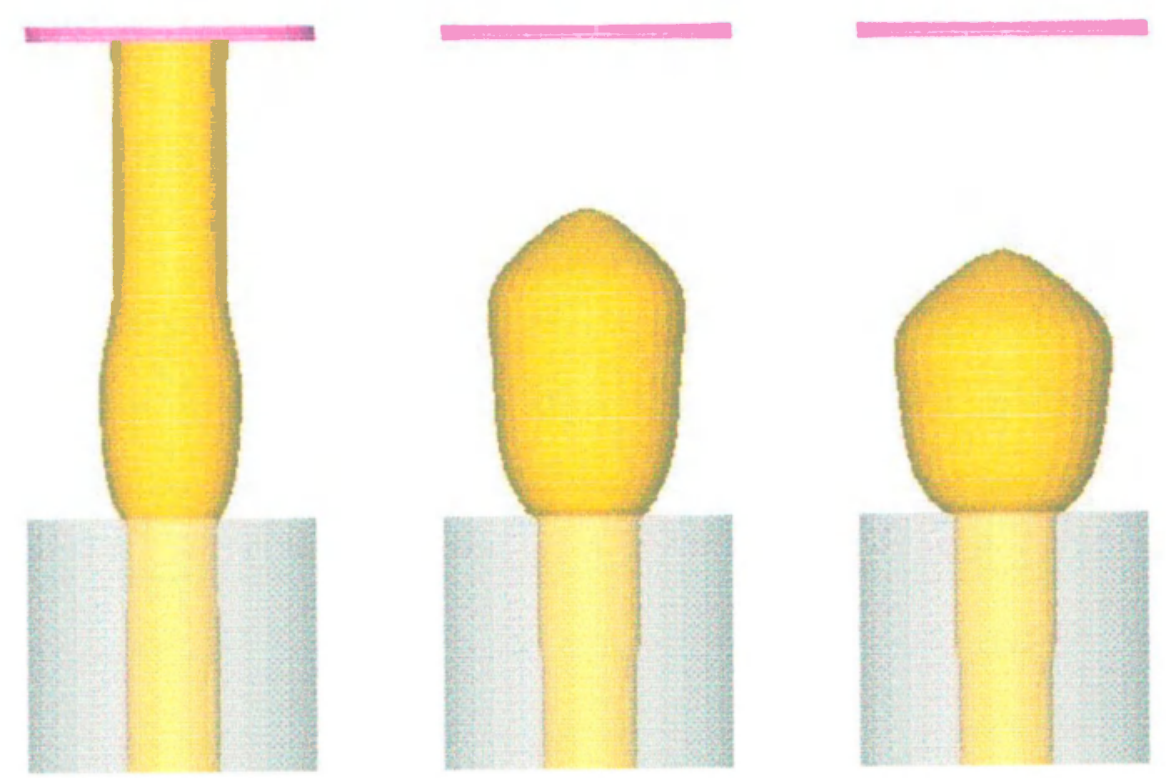

Figura 4.6: Continuação. 
$t=0.36 \mathrm{~s}$
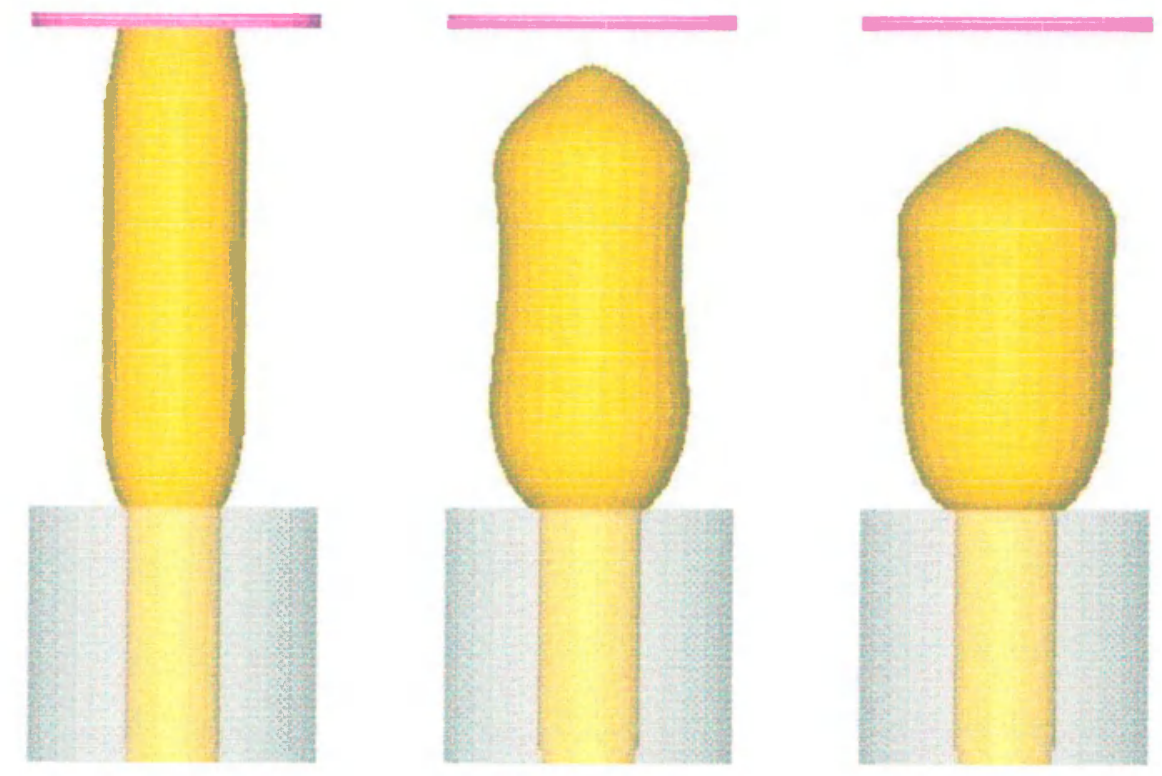

$t=0.72 \mathrm{~s}$
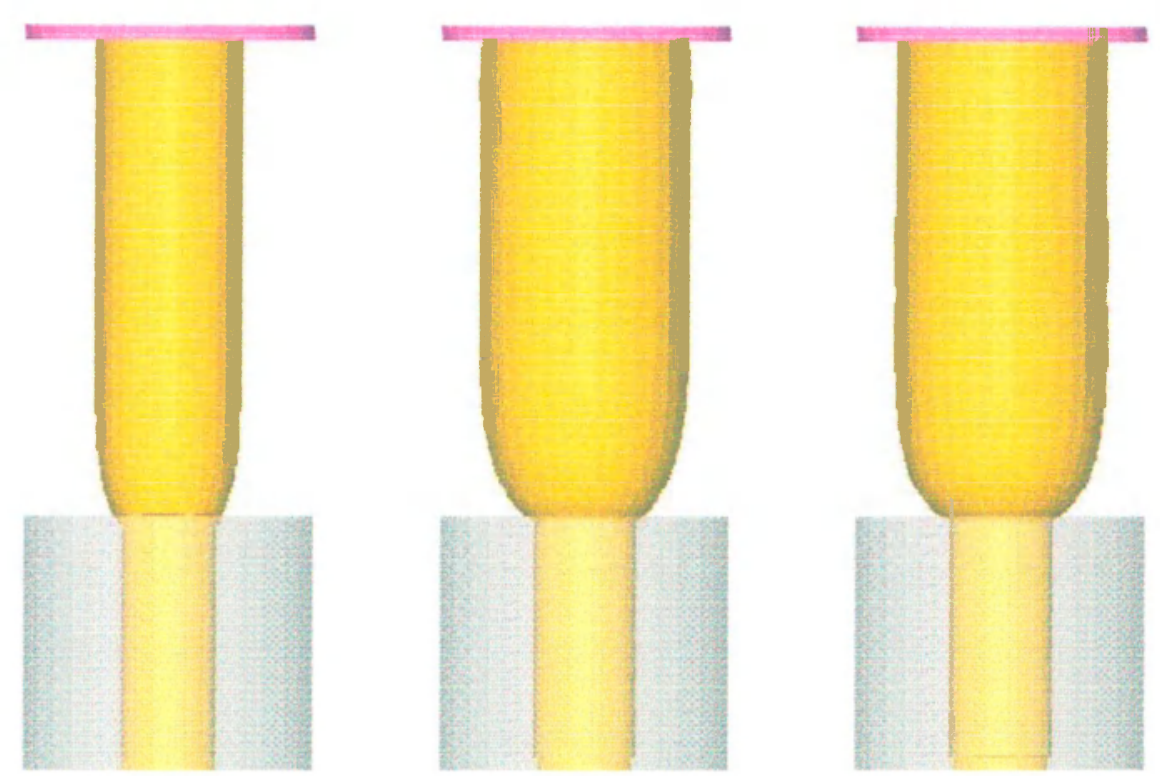

Figura 4.6: Continuação. 


$$
t=1.44 s
$$
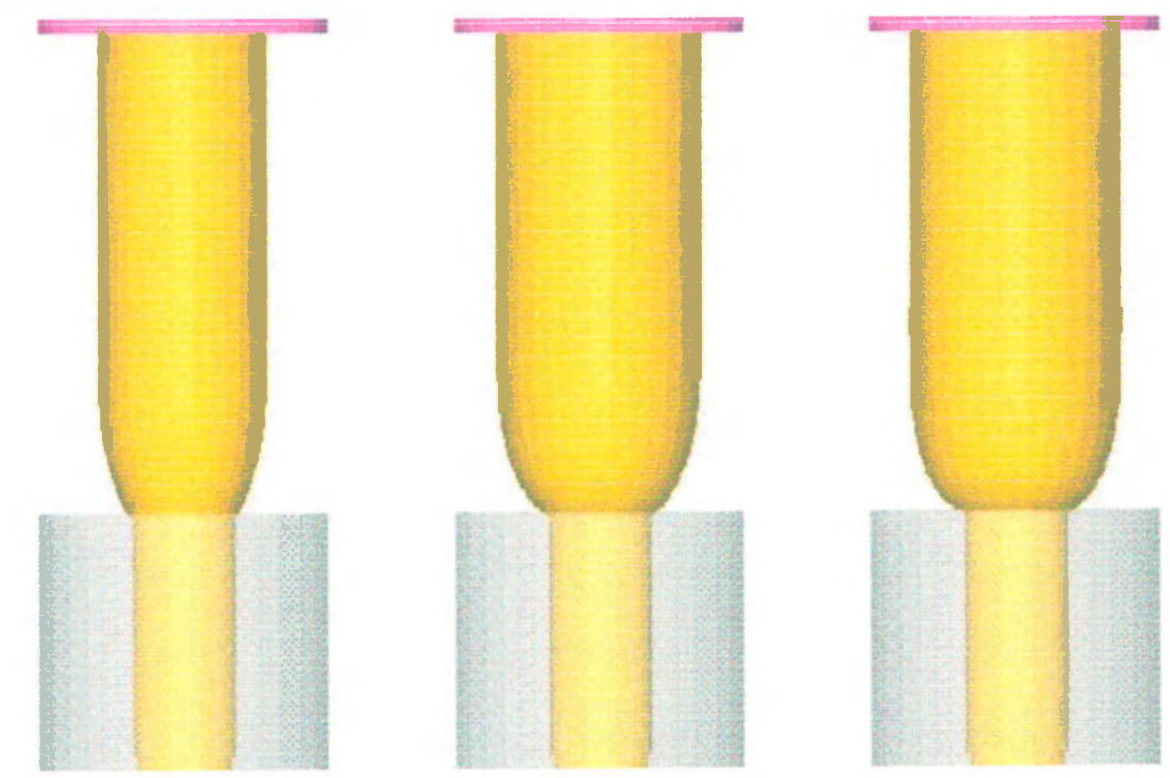

$t=2.0 \mathrm{~s}$
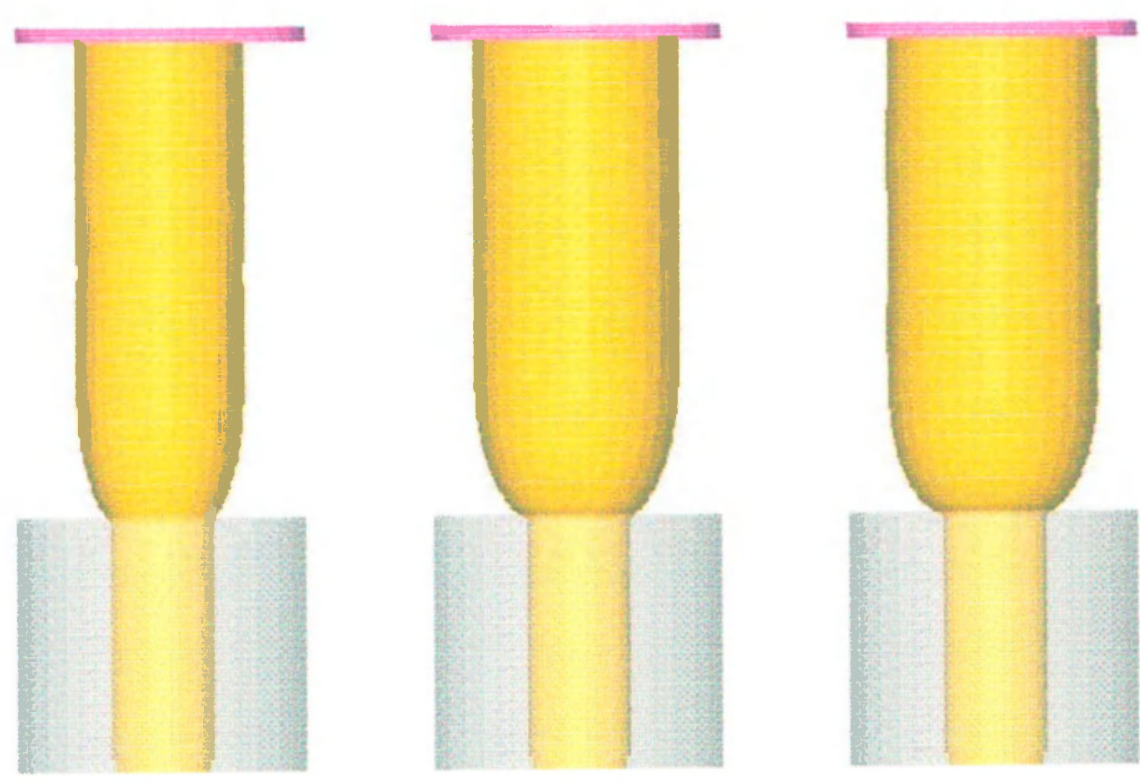

Figura 4.6: Continuação. 
Os resultados das simulaçōes mostrados na figura 4.5 foram utilizados para obtemos as componentes $\tau^{r r}, \tau^{r z}, \tau^{\theta 0}$ e $\tau^{z z}$ e as componentes de velocidades $u$ e $v$ no estado estacionário para $W e_{\text {effect }}=0.2,0.5$ e 0.8 .

l'or meio de uma análise sobre a solução analítica das componentes do tensor extratensão $\tau$ no estado estacionário dentro do tubo, as figuras $4.7-4.14$ mostram que, no eixo de simetria (meio do tubo) a componente $\tau^{z z}$ se anula e cresce continuamente até a parede do tubo; a componente $\tau^{r z}$ também se anula no eixo de simetria e decresce até a parede do tubo e as componentes $\tau^{r r}$ e $\tau^{\theta \theta}$ são nulas. De fato. pelas mudança de variáveis utilizada (vor equação (1.7)), temos

$$
\begin{aligned}
\tau^{r r} & =S^{r r}+\frac{2}{R e}\left(\frac{\lambda_{2}}{\lambda_{1}}\right)\left(\frac{\partial u}{\partial r}\right), \\
\tau^{r z} & =S^{r z}+\frac{1}{R e}\left(\frac{\lambda_{2}}{\lambda_{1}}\right)\left[\left(\frac{\partial v}{\partial r}\right)-\left(\frac{\partial u}{\partial z}\right)\right], \\
\tau^{\theta \theta} & =S^{\theta \theta}+\frac{2}{R e}\left(\frac{\lambda_{2}}{\lambda_{1}}\right)\left(\frac{u}{r}\right), \\
\tau^{z z} & =S^{z z}+\frac{2}{R e}\left(\frac{\lambda_{2}}{\lambda_{1}}\right)\left(\frac{\partial v}{\partial z}\right) .
\end{aligned}
$$

Ltilizando a equação (4.2), obtemos (desde que $\frac{\partial u}{\partial z}=\frac{\partial u}{\partial r}=\frac{\partial v}{\partial z}=0$ )

$$
\begin{aligned}
\tau^{r r} & =0 \\
\tau^{r z} & =\frac{1}{R e}\left[\frac{\partial v}{\partial r}\right], \\
\tau^{\theta \theta} & =0 \\
\tau^{z z} & =S^{z z}=2 \frac{W e}{R e}\left(1-\frac{\lambda_{2}}{\lambda_{1}}\right)\left(\frac{\partial v}{\partial r}\right)^{2} .
\end{aligned}
$$

Assim,

$$
N_{1}=\tau^{z z}-\tau^{r r}=\tau^{z z}
$$

Utilizando as equaçós (1.6) - (4.7) podemos discutir alguns fatos. A componente $\tau^{z z}$ depende de $\left(\frac{\partial v}{\partial r}\right)^{2}$, que é zero no centro do tubo, onde a velocidade é máxima, depois aumenta até atingir a parede do tubo. A componente $\tau^{r z}$ depende de $\frac{\partial v}{\partial r}$, que também é zero no centro do tubo, onde a velocidade é máxima, depois decresce até atingir a parede. As componentes $\tau^{r r}$ e $\tau^{\theta \theta}$ são zero em todo o tubo, onde podemos concluir que $N_{1}=\tau^{z z}$. O valor de $N_{1}$ é importante, pois á responsável pelo inchamento do extrudado: quanto maior for o valor de $N_{1}$ maior será o inchamento do extrudado.

Na saída do tubo o fluido passa por uma transição devido a mudança no tipo de condição de contorno (altamente cisalhante para condição de superfície livre) e depois ocorre o fenômeno de relaxação, não tendo mais taxa de cisalhamento e obtendo as variáveis em questões nulas. 

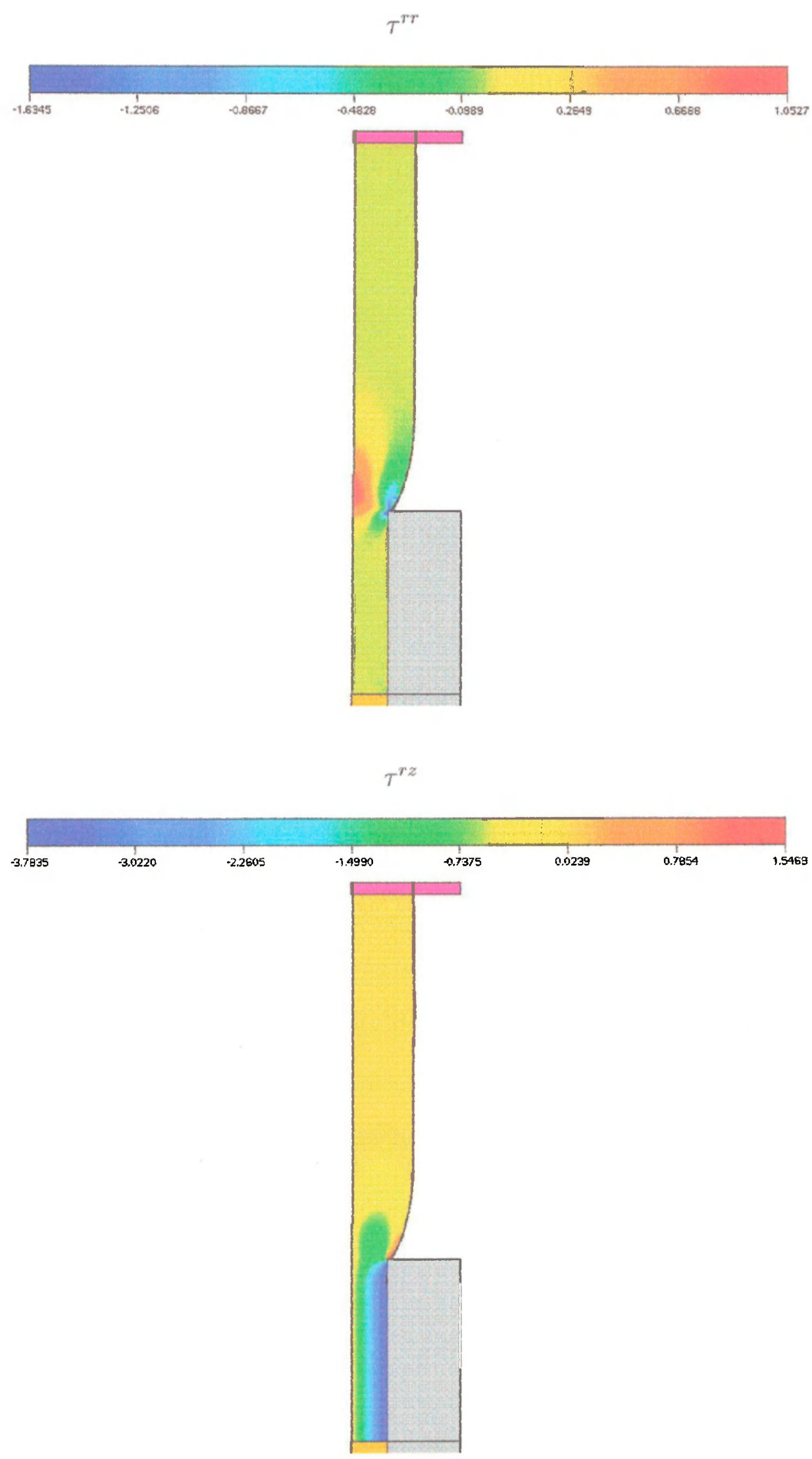

Figura 4.7: Visualização bidimensional das componentes $\tau^{r r}$ e $\tau^{r z} . W e_{\text {effect }}=0.2 \mathrm{e}$ lempo $t=100 \mathrm{~s}$ (estado estacionário). 


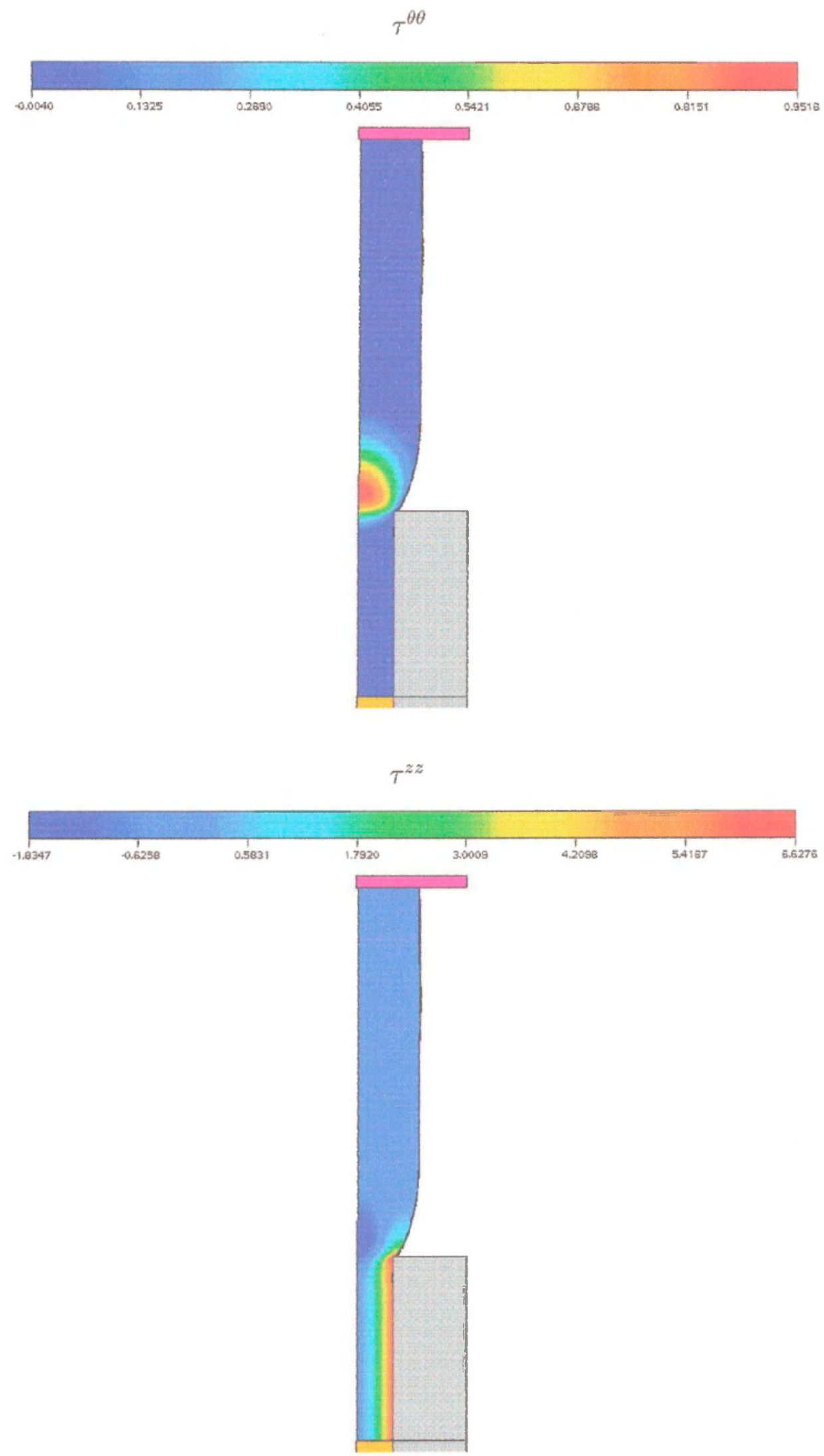

Figura 4.8: Visualização bidimensional das componentes $\tau^{\theta\urcorner} \in \tau^{z z}$. W effect $=0.2$ e tempo $t=100 \mathrm{~s}$ (estado estacionário). 

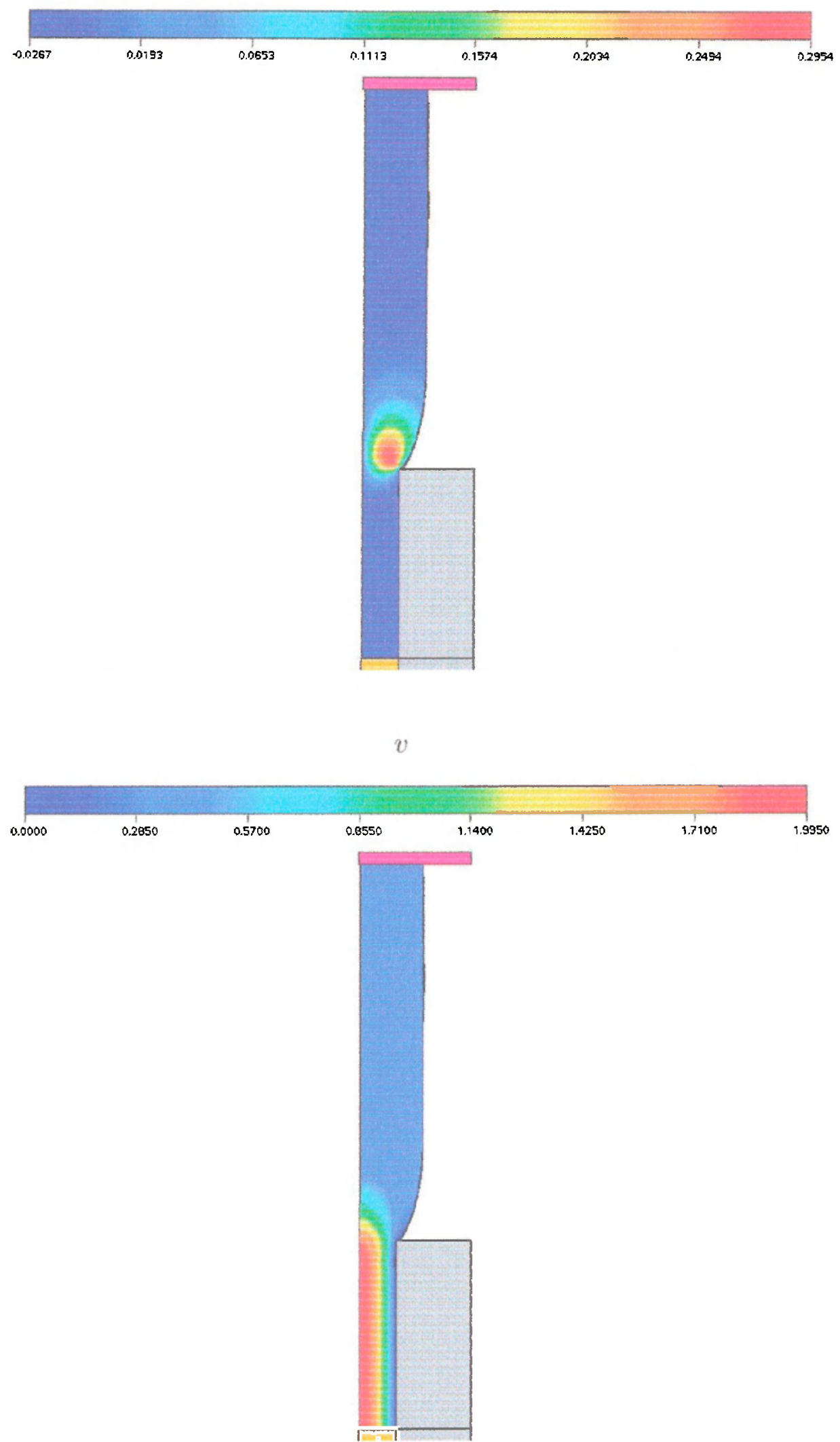

Figura 4.9: Visualização bidimensional das componentes da velocidade $u$ e $v . W e_{\text {eflect }}=0.2$ e tempo $t=100 \mathrm{~s}$ (estado estacionário). 

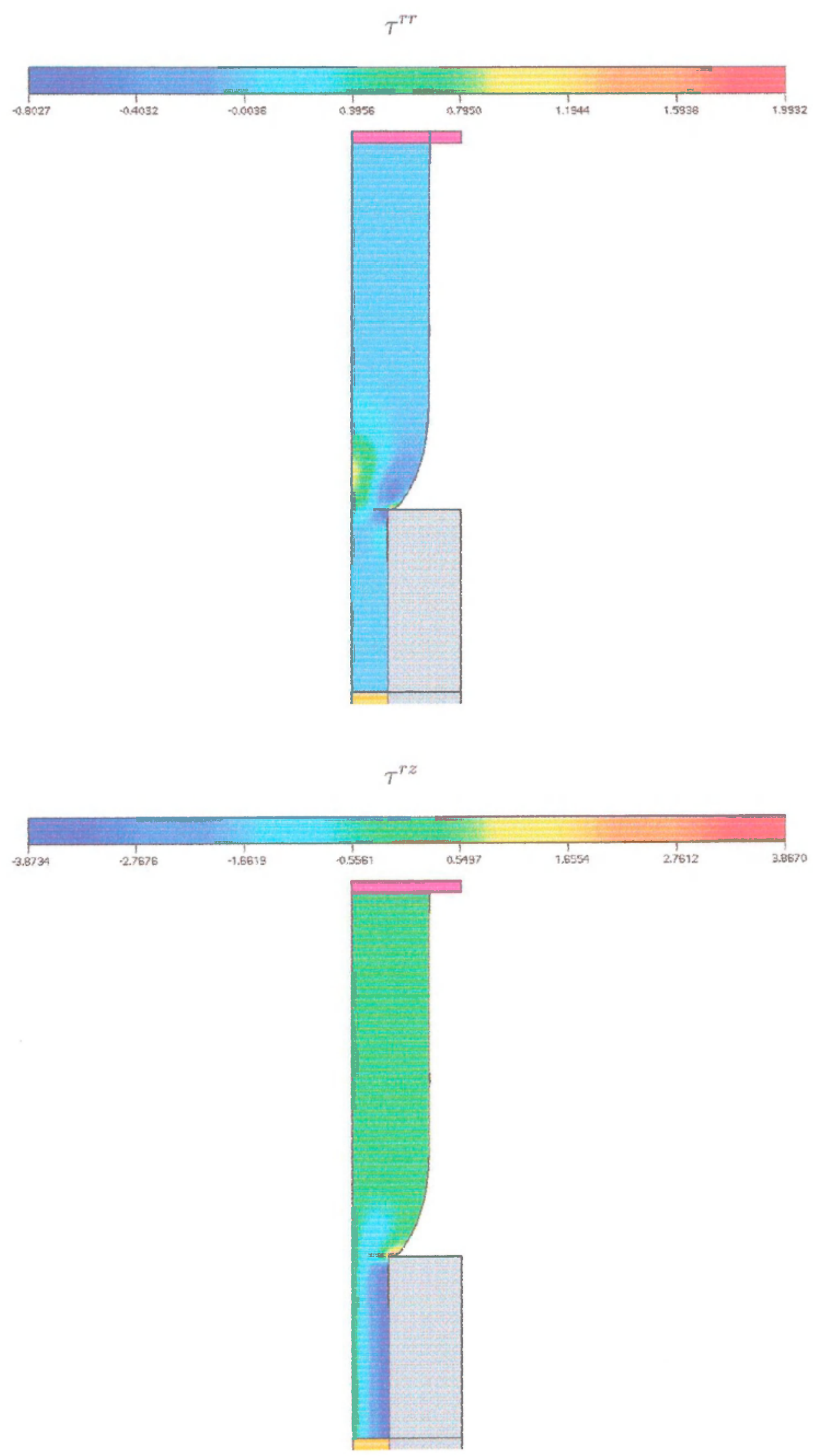

Figura 4.10: Visualização bidimensional das componentes $\tau^{T \tau}$ e $\tau^{\tau z} \cdot W e_{\mathbf{e}}$ ect $=0.5 \mathrm{e} \mathrm{tempo}$ $t=100 \mathrm{~s}$ (estado estacionário). 

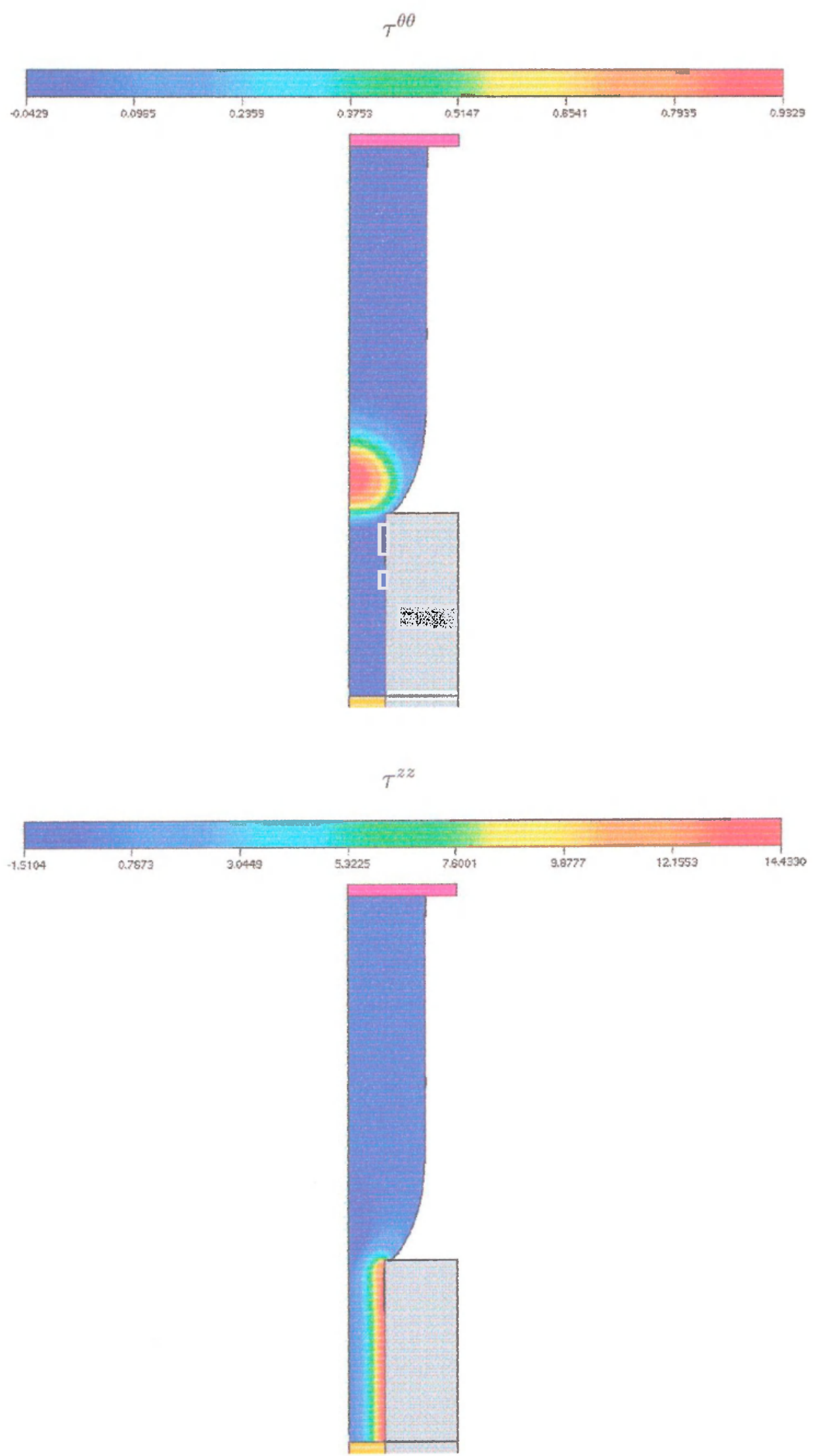

Figura 4.11: Visualização bidimensional das componentes $\tau^{96}$ e $\tau^{z z} . W e_{\mathbf{e}}$ ecl $=0.5$ e tempo $t=100 s$ (estado estacionário). 
u

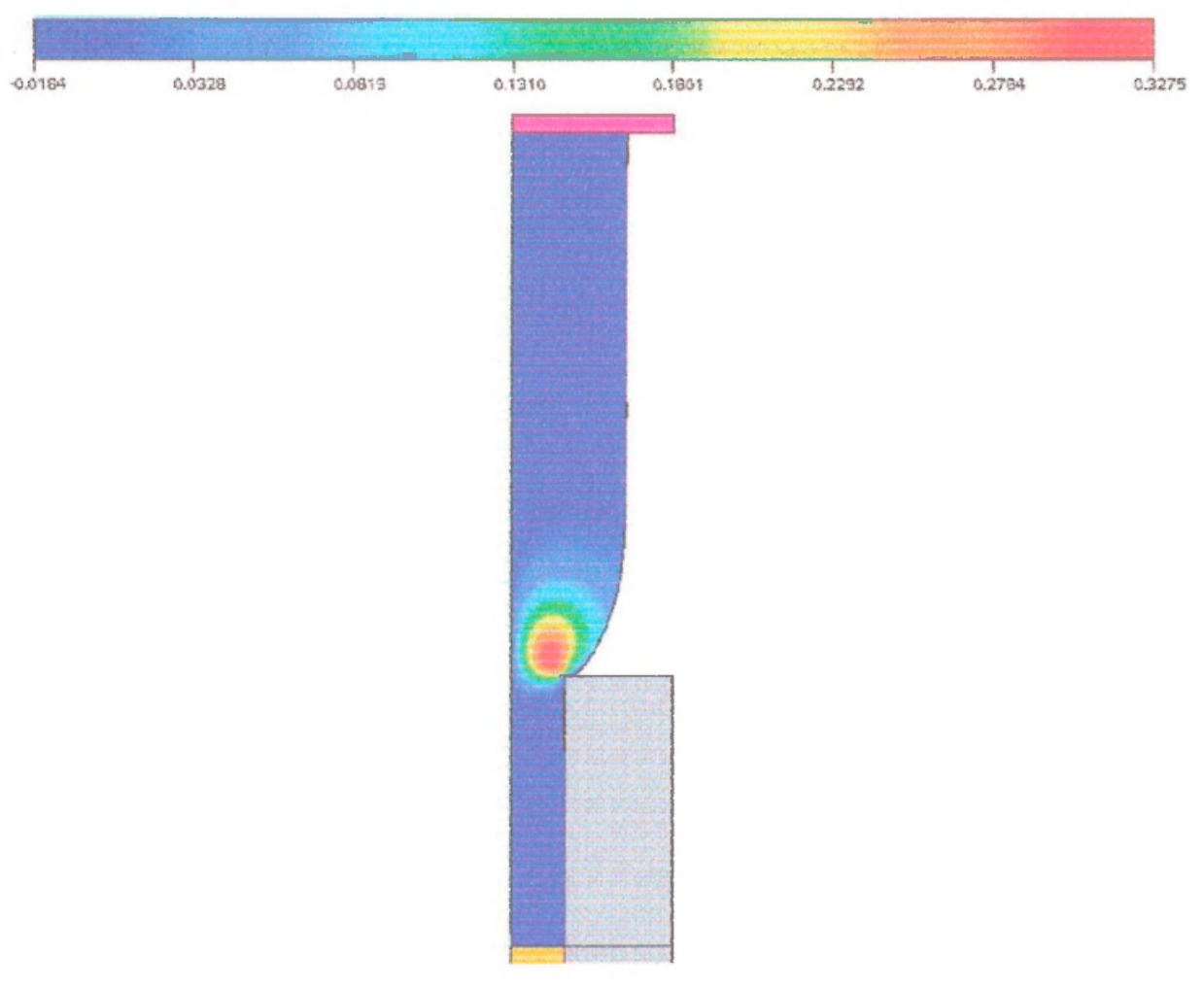

$v$

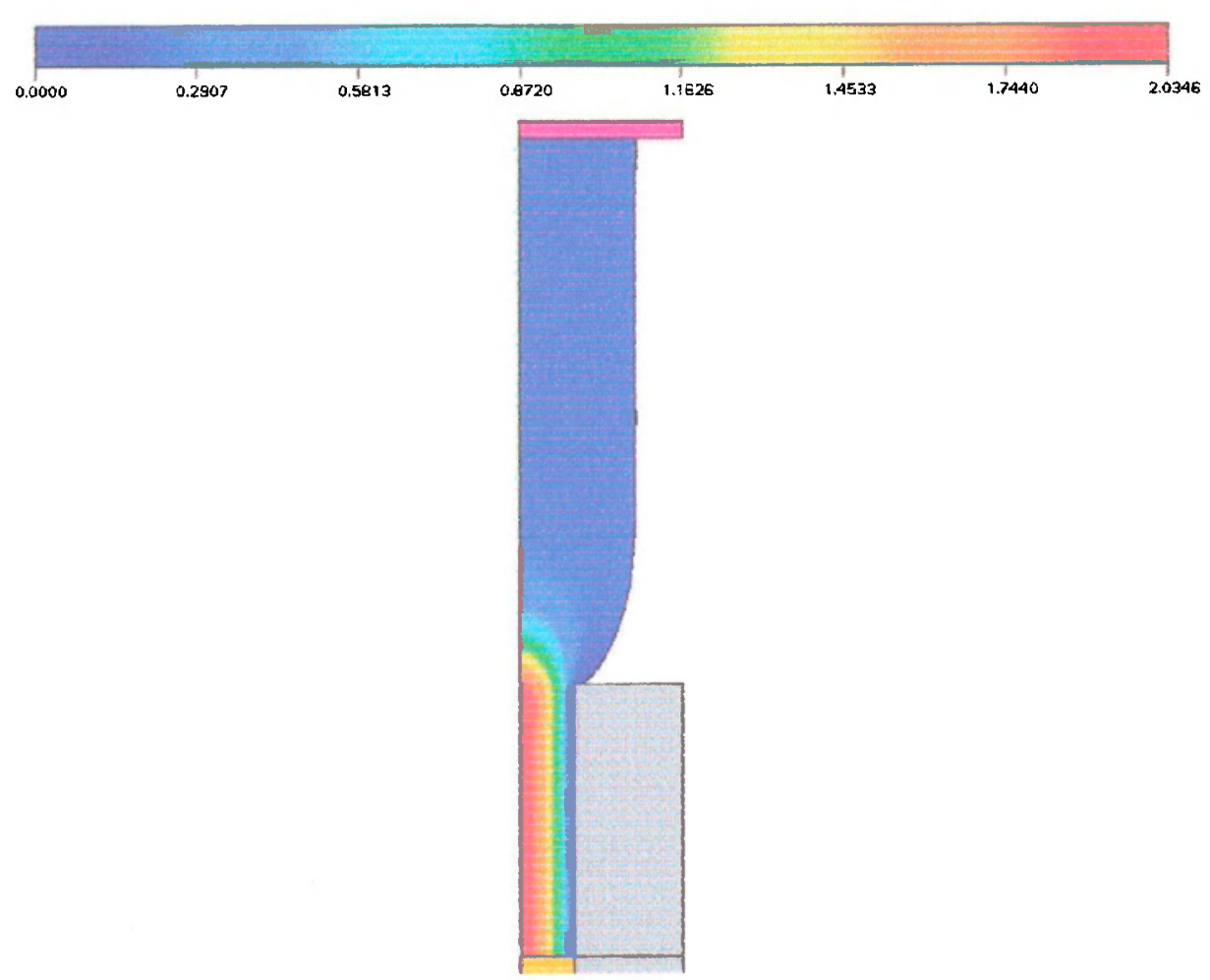

Figura 4.12: Visualização bidimensional das componentes da velocidade $u$ e $v$. $W e_{\text {effect }}=$ 0.5 e tempo $t=100$ s (estado estacionário). 


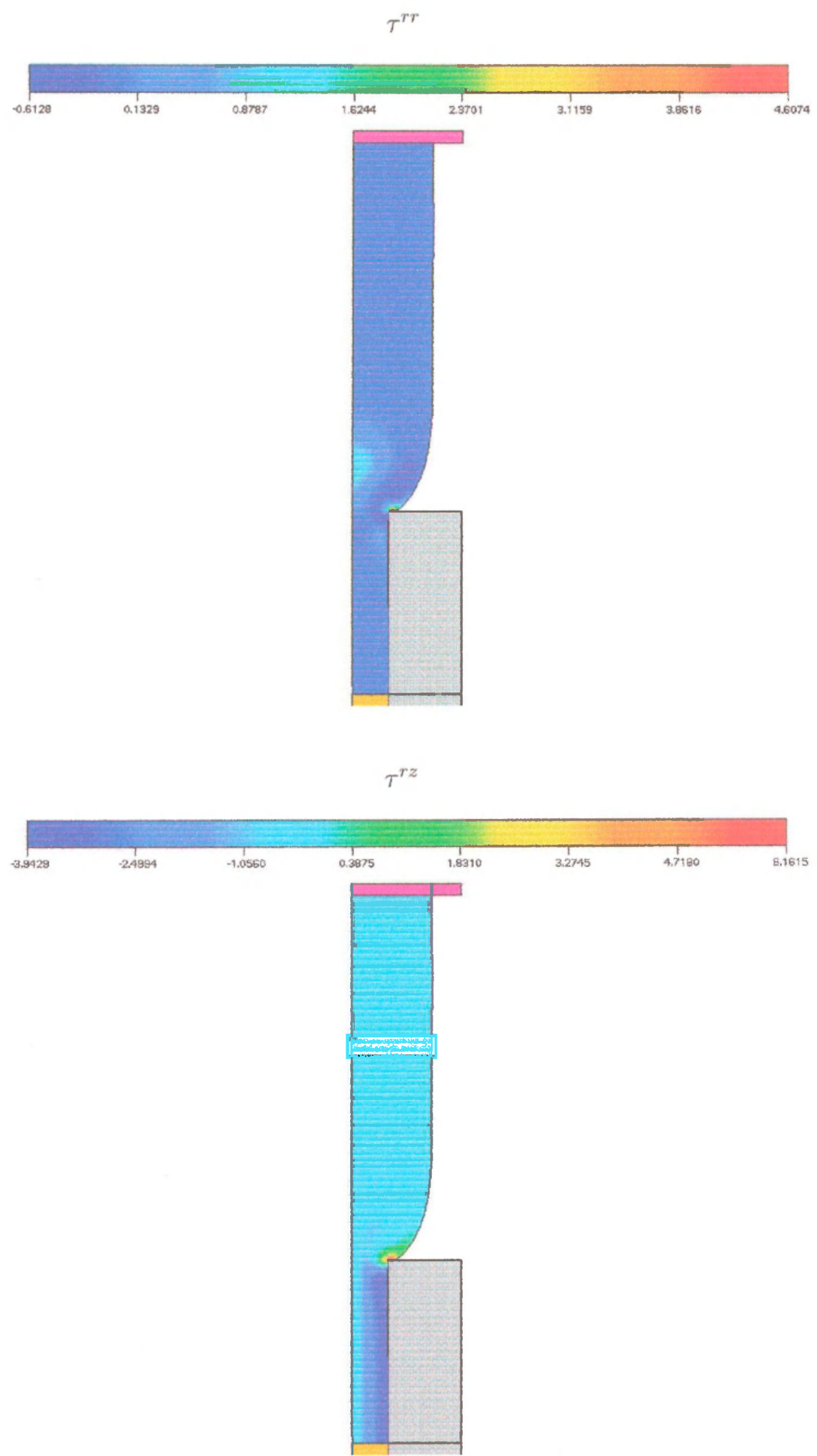

Figura 4.13: Visualização bidimensional das componentes $\tau^{r r}$ e $\tau^{r z} \cdot W e_{\text {effect }}=0.8$ e tempo $t=100 \mathrm{~s}$ (estado estacionário). 

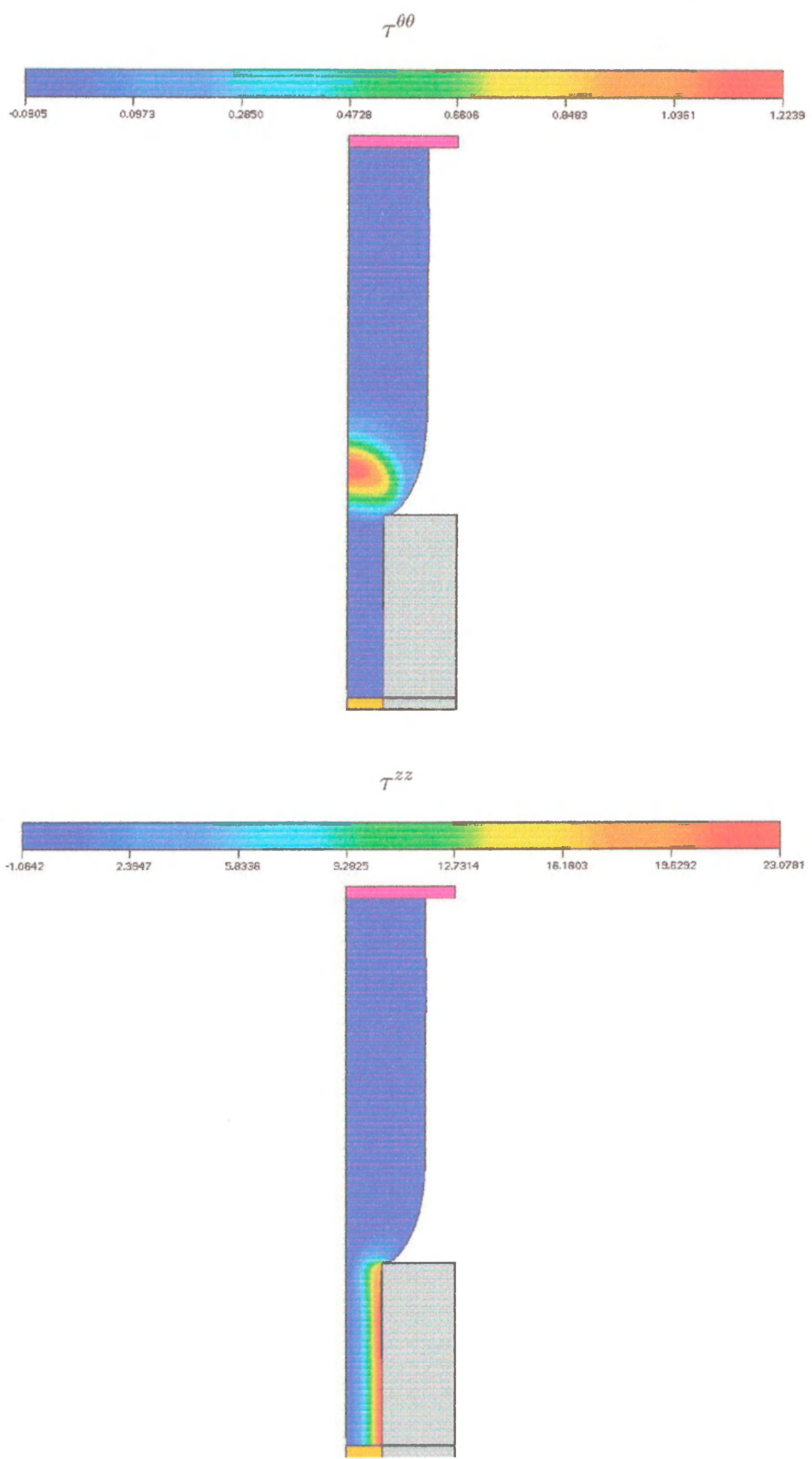

Figura 4.14: Visualização bidimensional das componentes $\tau^{6 \theta} \in \tau^{z z} . W e_{\text {effect }}=0.8$ e tempo $t=100 \mathrm{~s}$ (estado estacionário). 


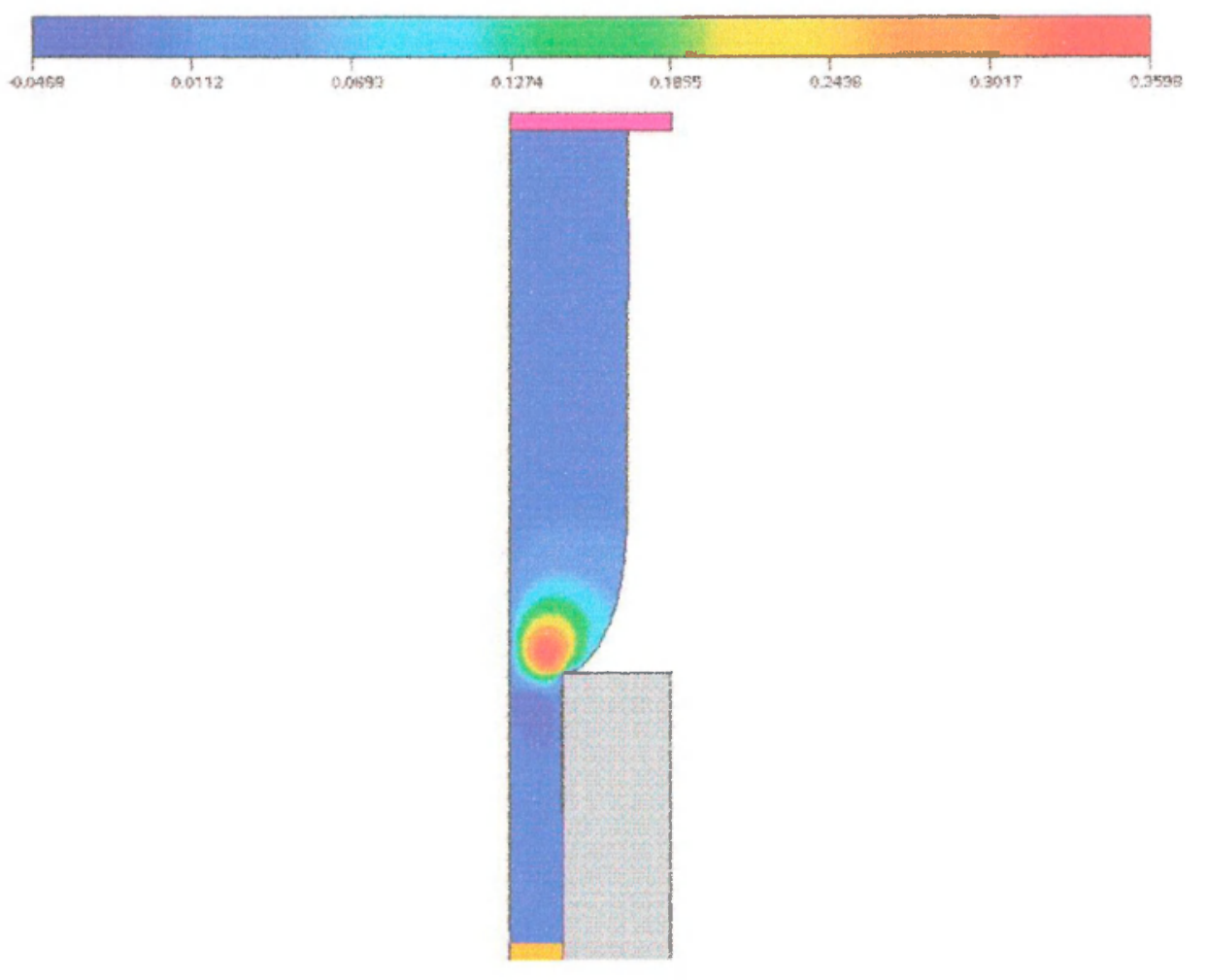

$v$

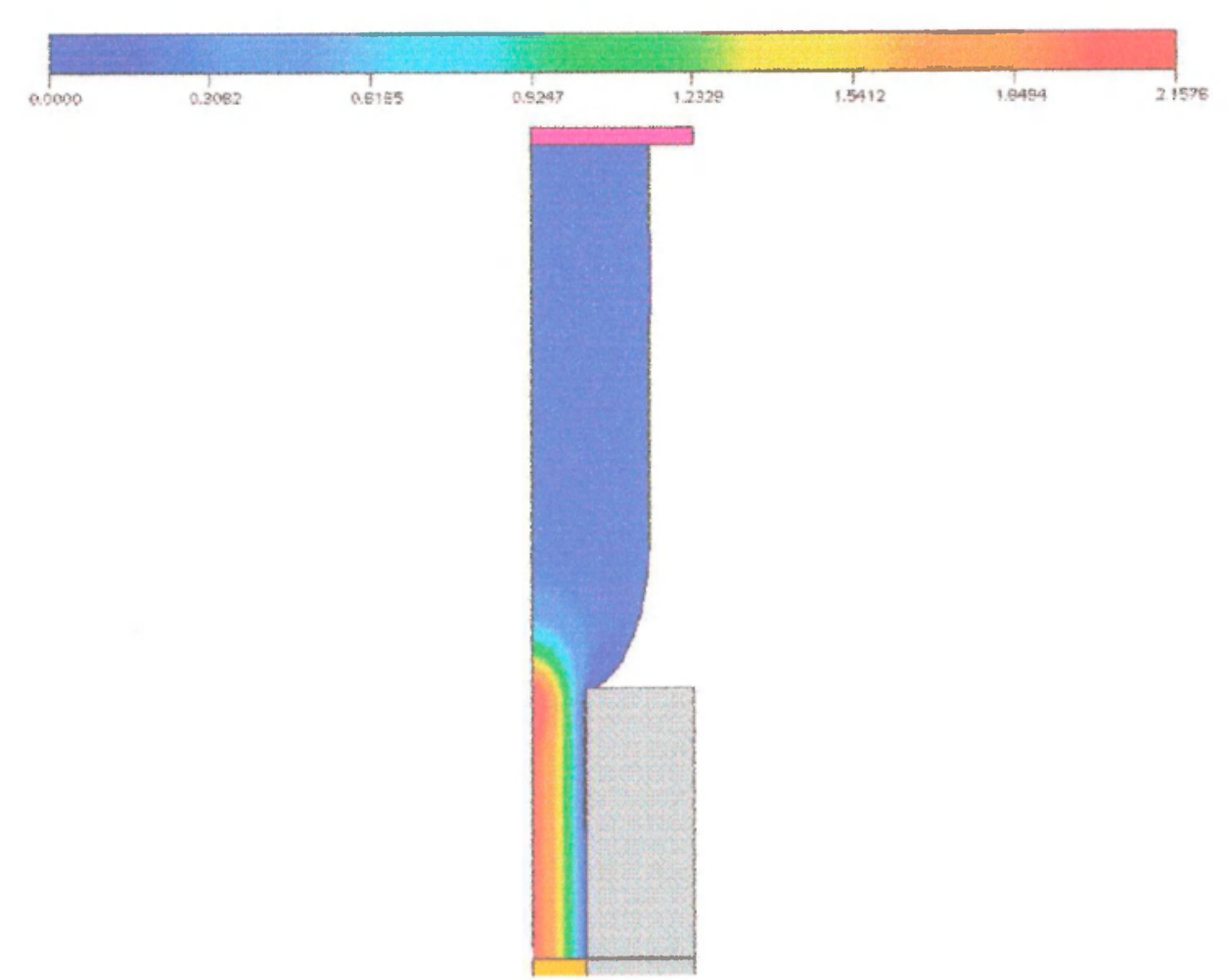

Figura 4.15: Visualização bidimensional das componentes da velocidade $u$ e $v$. We efrect $=$ 0.8 e tempo $t=100$ s (estado estacionário). 


\subsection{Simulação Numérica do Impacto de uma Gota con- tra uma Superfície Rígida}

Nesta seção apresentamos os resultados numéricos da simulação de uma gota axissimétrica contendo fluido Oldroyd-B incidindo contra uma superfície rigída (ver figura 4.16). Os efeitos viscoelásticos podem ser observados comparando os resultados obtidos por meio da simulação de uma gota contendo fluido viscoelástico com os resultados da simulação de uma gota contendo fluido newtoniano.

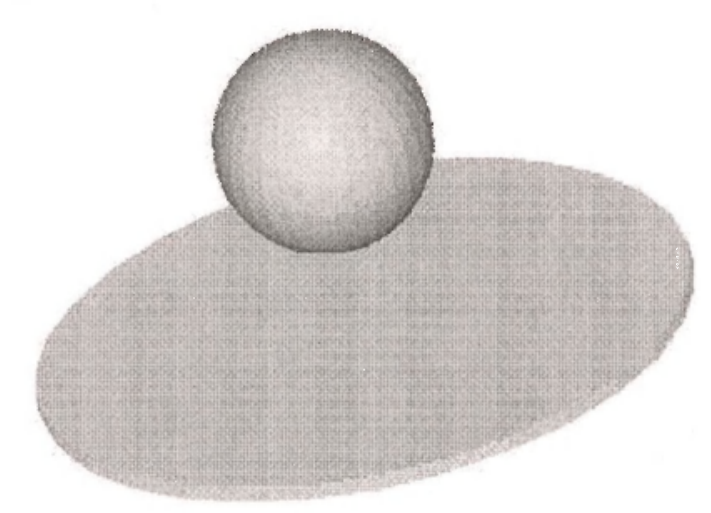

Figura 4.16: Descrição do domínio computacional para a simulação de uma gota incidindo contra uma superfície rígida.

Para simular este problema, impomos a condição de não-escorregamento para a velocidade na superfície rígida e para as componentes do tensor extra-tensão foram impostas as condições apresentadas nas seções 3.9 e 3.10. Na superfície livre aplicamos as condições de tensão normal e tensão tangencial nulas (ver equações 1.62 e 1.63), conforme as equações de diferenças finitas descritas na seção 3.8 .

Os seguintes dados de entrada foram utilizados:

- Domínio Computacional: $0.003 \mathrm{~m} \times 0.003 \mathrm{~m}$;

- Espaçamento da malha: $\delta r-\delta z=0.0005 \mathrm{~m}(60 \times 60$ células $)$

- Velocidade inicial da gota $U=-1.0 \mathrm{~ms}^{-1}$;

- Diâmetro da gota $D=1 \mathrm{~cm}$;

- Viscosidade cinemática: $\nu=0.002 \mathrm{~m}^{2} \mathrm{~s}^{-1}$

- Definição do modelo Oldroyd-B: $\lambda_{1}=0.01 \mathrm{~s}, \lambda_{2}=0.001 \mathrm{~s}$;

- Parâmetros de escala: $D, U, \nu, \lambda_{1}$. Logo, $R e=U D / \nu=5.0$ e $W e=\lambda_{1} \frac{U}{D}=1.0$ e $\lambda_{2} / \lambda_{1}=0.1$ 
A diferença no comportamento da gota de fluido contendo Oldroyd-B e da gota contendo fluido newtoniano é caracterizada pela mudança na deformação das gotas no decorrer do escoamento. As figuras 4.17 - 4.18 mostram o perfil de $u$ e $v$ para vários tempos.

Os resultados referentes a gota contendo fluido newtoniano mostram que a mesma escoa monotonicamente na direção radial (como podemos ver pelo sinal sempre positivo da velocidade $u$ ) e exibe uma redução contínua das velocidades até alcançar sua forma deformada final. Por outro lado, os resultados referentes a gota contendo fluido Oldroyd-B mostram que, inicialmente a gota escoa na direção radial exibindo uma redução contínua das velocidades, mas entre os tempos $0.018 \mathrm{~s}$ e $0.3 \mathrm{~s}$, ocorre a inversão do sinal das velocidades indicando um movimento contrário ao inicial e a gota sofre o efeito de uma contração. Esse efeito é típico do material clástico e foi obtido em simulações realizadas por L. Grossi [11] e Tomé et al. [12]. As taxas de deformação e as velocidades diminuem com o passar do tempo até o fluido Oldroyd-B aproximar-se da sua deformação final. Se houver ainda alguma tensão no fluido, o fluido passa a relaxar essa tensão, aumentando a taxa de deformação e as velocidades, iniciando um novo ciclo de deformação na direção oposta. A cada ciclo, a amplitude da deformação alcançada diminui em função da energia dissipada pelo fluido. Esta é a explicação para o aspecto elástico da deformação da gota contendo fluido Oldroyd-B. 


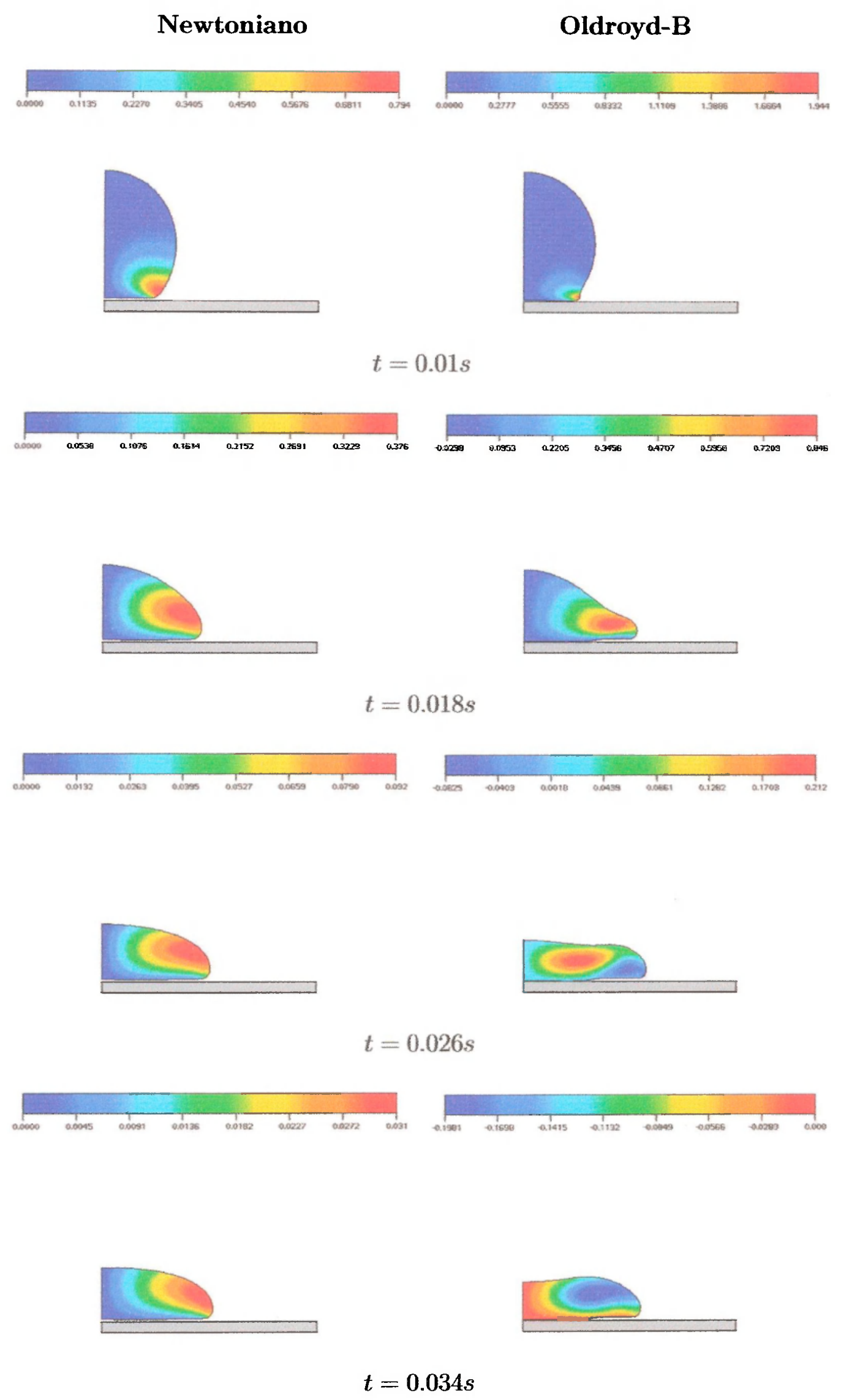

Figura 4.17: Visualização das curvas de nível da velocidade $u$ em diferentes tempos. 

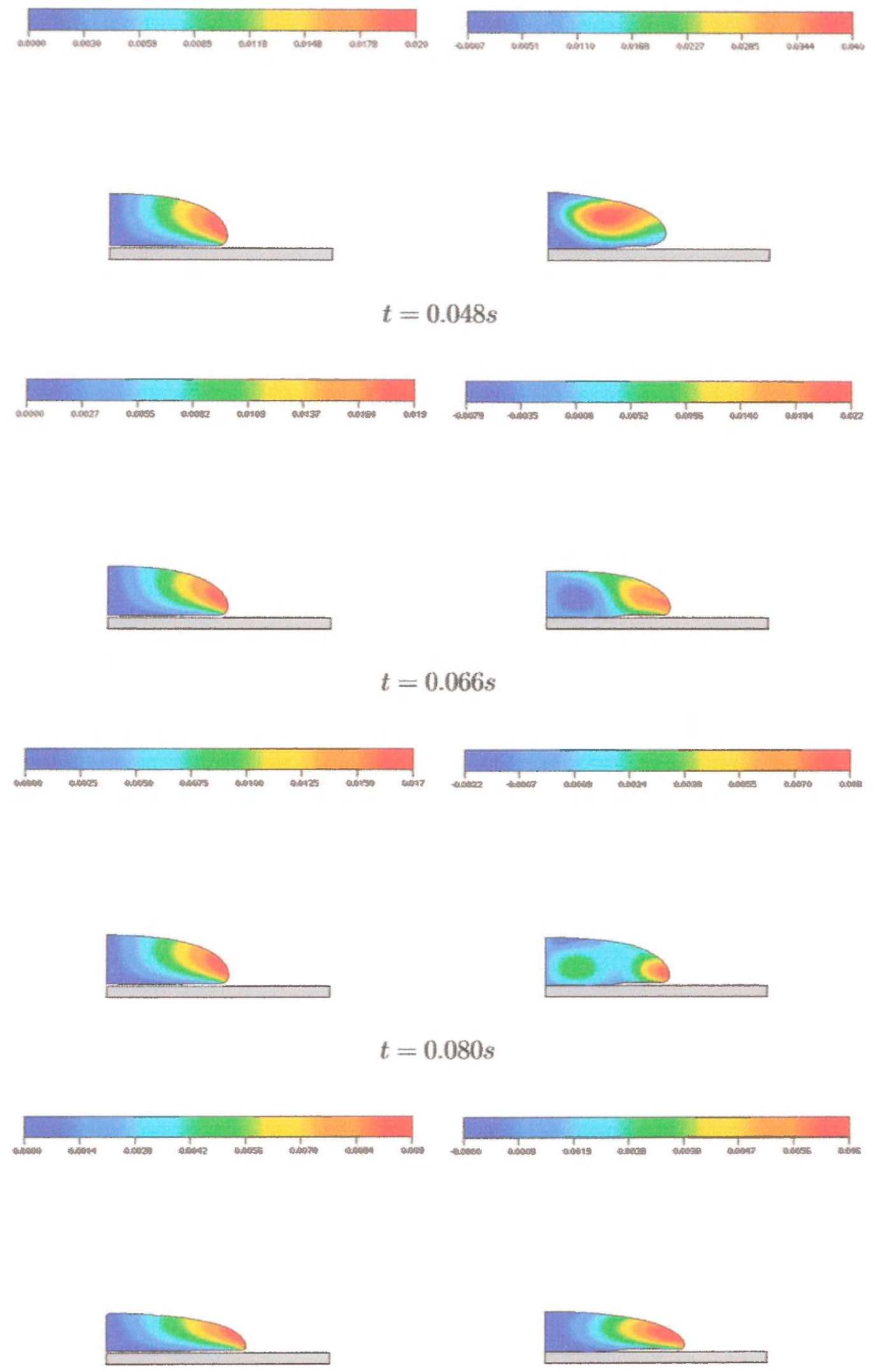

$$
t=0.30 s
$$

Figura 4.17: Continuação. 

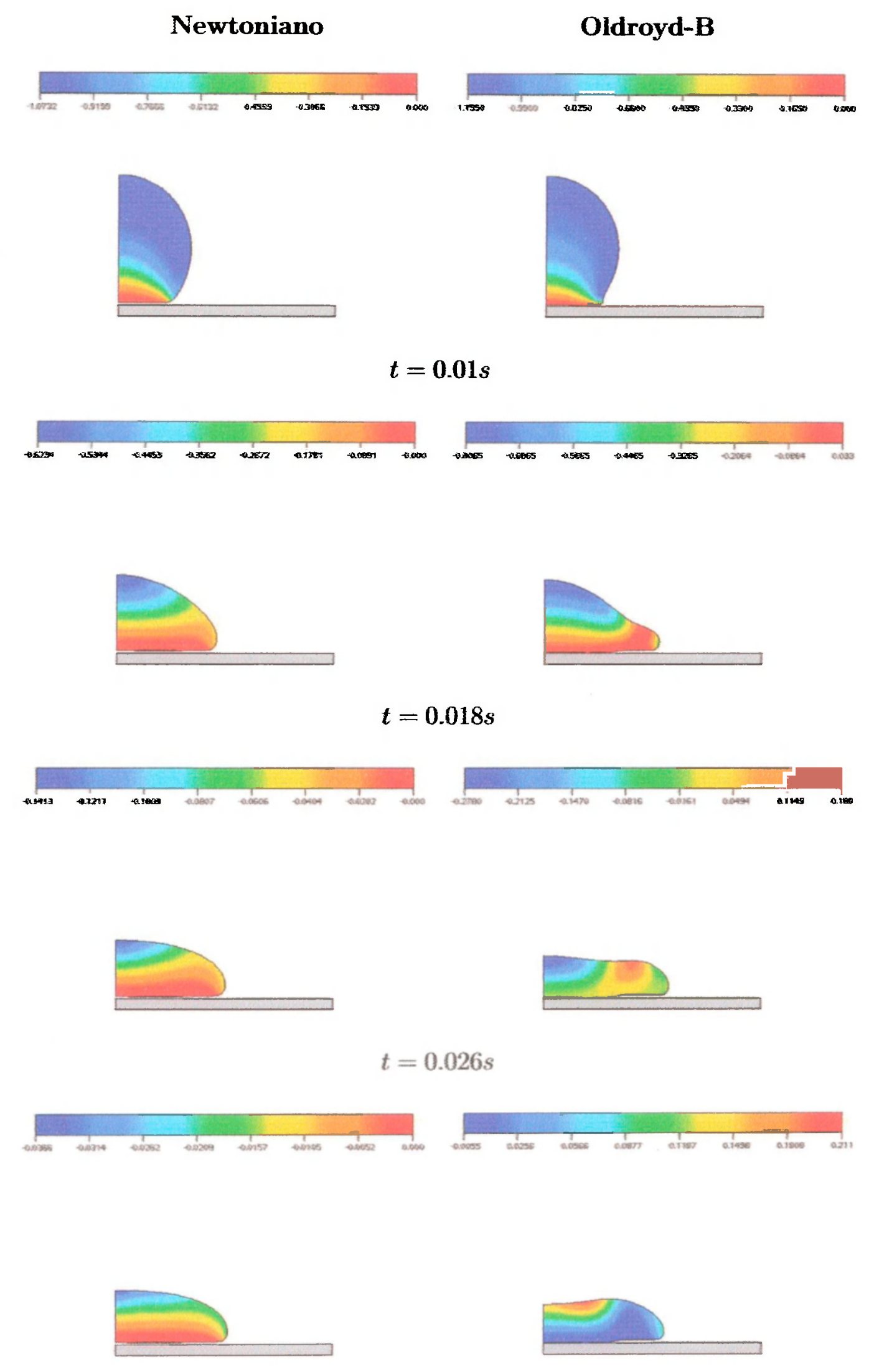

$t=0.034 s$

Figura 4.18: Visualização das curvas de nível da velocidade $v$ em diferentes tempos. 

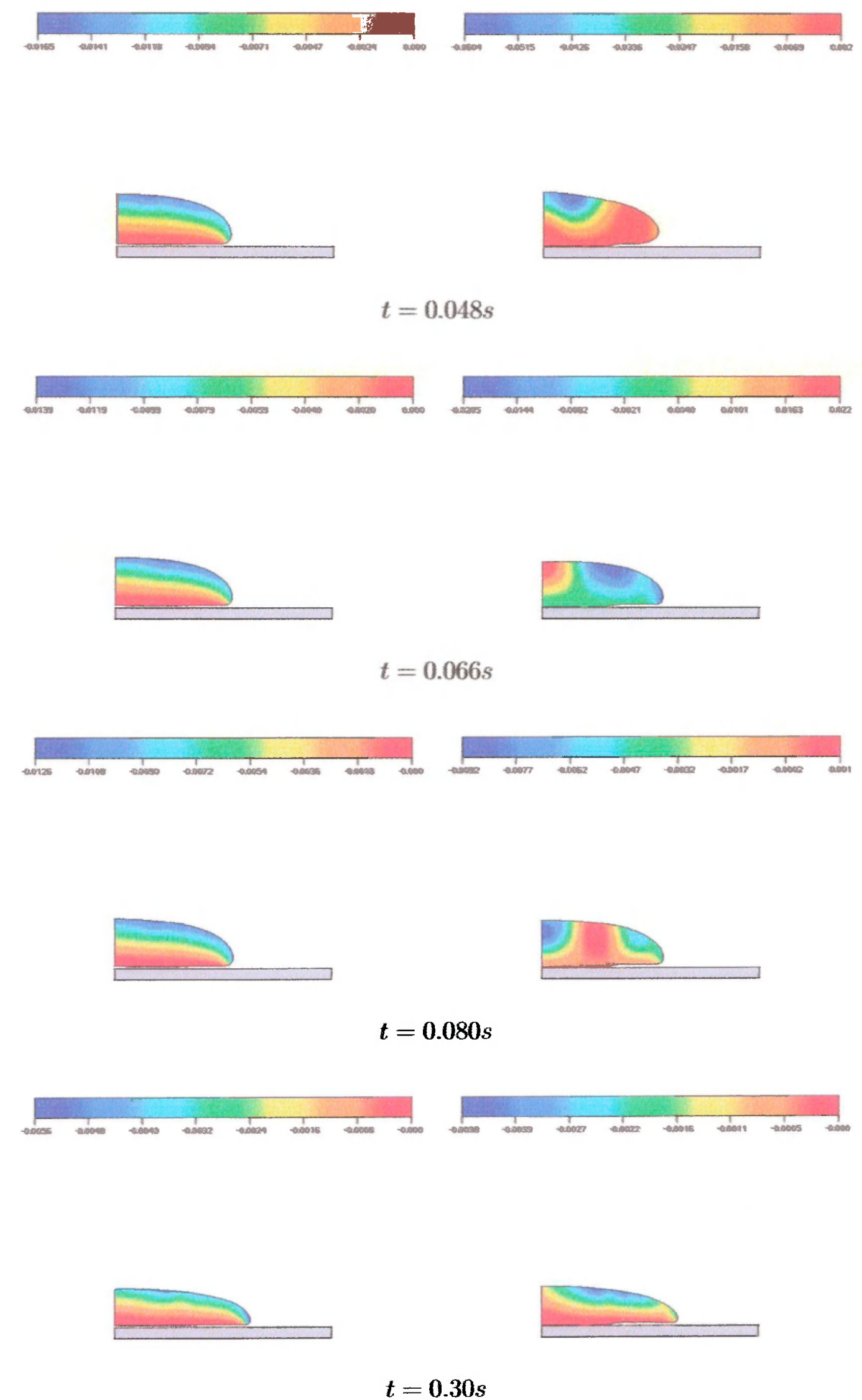

Figura 4.18: Continuação. 


\subsection{Simulação Numérica do 'splashing drop'}

Quando uma esfera líquida cai de uma certa altura em uma piscina contendo o mesmo fluido, uma sequência de fenômenos pode ocorrer: uma cratera c um cume podem se formar seguidos de um 'splash' (ver figura 4.20; um esquema de representação é dado na figura 4.19). Esse fenômeno foi primeiramente investigado por Worthington numa série de artigos ([23], [24]) e por último num livro [25]. Recentemente tem surgido um maior interesse no que agora é conhecido como 'Worthington jet'. Um grupo de pesquisadores da universidade de Wales em Aberystwyth tem estudado esse fenômeno numa sequência de artigos ([26], [27], [28]). Uma de suas descobertas mais interessantes é que se um pouco menos de $10 \mathrm{ppm} / \mathrm{g}$ de polímero for adicionado a um fluido newtoniano então o 'splash' pode ser reduzido mais que uma ordem de magnitude. Na verdade, o interesse deles e sem dúvida, o interesse de Worthigton foi com esferas de vidro ou metal caindo num líquido parado e certamente neste caso, a redução na altura do 'Worthington jet' é dramática. Este trabalho se restringirá ao caso de uma esfera líquida caindo cm um tanque com o mesmo fluido a qual foi também estudada experimentalmente por Cheny c Walters [26]. Esses autores mostraram que a

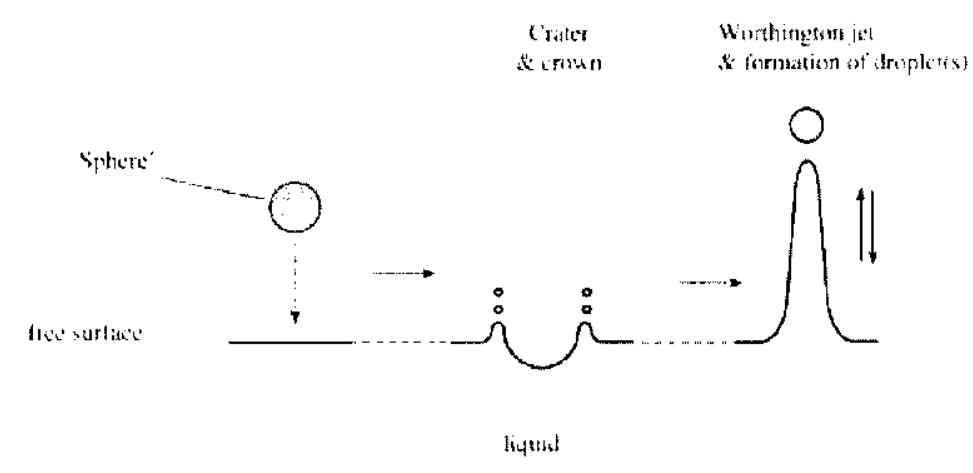

Figura 4.19: Esquema de representação para o problema do splashing drop.

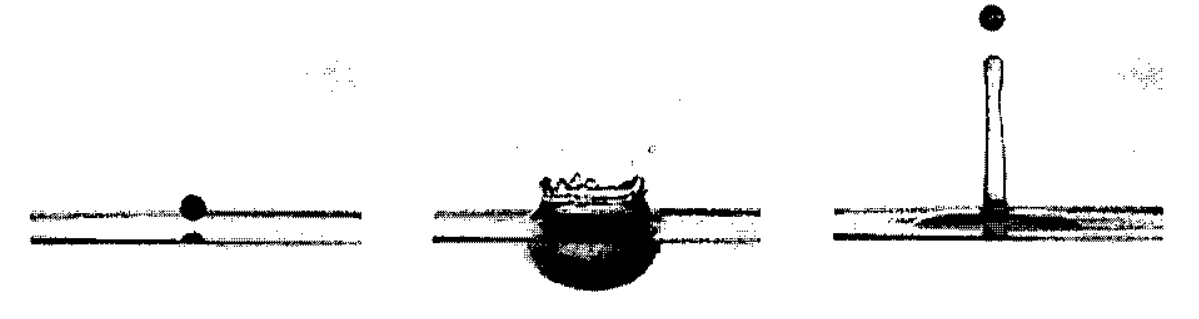

Figura 4.20: Observações experimentais do fenômeno splashing drop.

adição de 10 wppm de 'polyacrylamide' também causou uma diminuição na altura, embora essa diminuição não foi tão dramática quanto foi com as esferas de vidro ou metálicas.

A seguir, apresentamos os resultados numéricos de várias simulações do 'splashing drop' para um fluido Oldroyd-B para diferentes números de Weissenberg e vários números de Reynolds. 


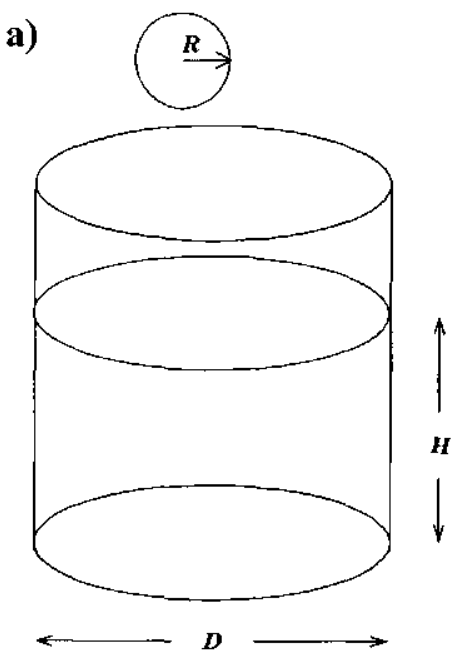

b)

Figura 4.21: Simulação numérica do 'splashing drop': domínio computacional.

Consideramos uma gota esférica de um fluido caindo num tanque cilíndrico (ver figura 4.21a). Assumimos escoamento axissimétrico e um domínio computacional como o mostrado na figura $4.21 \mathrm{~b}$. Os seguintes dados de entrada foram utilizados:

- Domínio Computacional: $0.1 \mathrm{~m} \times 0.2 \mathrm{~m}$;

- Espaçamento da malha: $\delta r=\delta z=0.001 \mathrm{~m}(100 \times 200$ células $)$;

- Velocidade de impacto da gota: $U=2.0 \mathrm{~ms}^{-1}$;

- Raio da gota: $L=0.005 \mathrm{~m}$;

- Diâmetro do tanque: $D=0.2 \mathrm{~m}$;

- acceleração gravitacional: $g_{r}=0, g_{z}=-9.81 \mathrm{~ms}^{-2}$;

- Parâmetros de escala: $U, L, \nu$ :

- Simulaçãa 1: Viscosidade cinemática $\nu=0.00002 \Longrightarrow R e=\frac{U L}{\nu}=500$

- Simulação 2: Viscosidade cinemática $\nu=0.00001 \Longrightarrow R e=\frac{\nu}{\nu}=1000$

- Simulação 3: Viscosidade cinemática $\nu=0.000005 \Longrightarrow R e=\frac{U L}{\nu}=2000$

Para observarmos o comportamento viscoelástico, apresentamos várias simulações aumentando o número de Weissenberg efetivo $W$ eeffect. (ver equação (4.4)). Yestas simulações estamos interessados em observar a influĉncia da viscoelasticidade (representada por $\left.W e_{\text {effect }}\right)$ na altura do 'Worthington jet'. Resultados experimentais têm mostrado que a altura do 'Worthington jet' deve diminuir devido ao aumento dos efeitos viscoelásticos.

Para cada número de Reynolds fizemos simulaçōes com We $=0.5\left(\lambda_{\mathrm{I}}=0.00125\right) \mathrm{c}$ $W e=1.0\left(\lambda_{1}=0.0025\right)$. Os valores do parâmetro $\lambda_{2}$ foram obtidos de maneira que a 
razão $\frac{\lambda_{2}}{\lambda_{1}}$ assumisse os valores $0.9,0.5$ e 0.3 . Desta forma, para $W e=0.5$, obtivemos $W e_{\text {effect }}=0.05,0.25$ e 0.35 e para $W e=1.0$ o número de Weissenberg efetivo tomou os valores $W e_{\text {effect }}=0.1,0.5$ e 0.7 . Para a simulação do fluido newtoniano fizemos $\frac{\lambda_{2}}{\lambda_{1}}=1$.

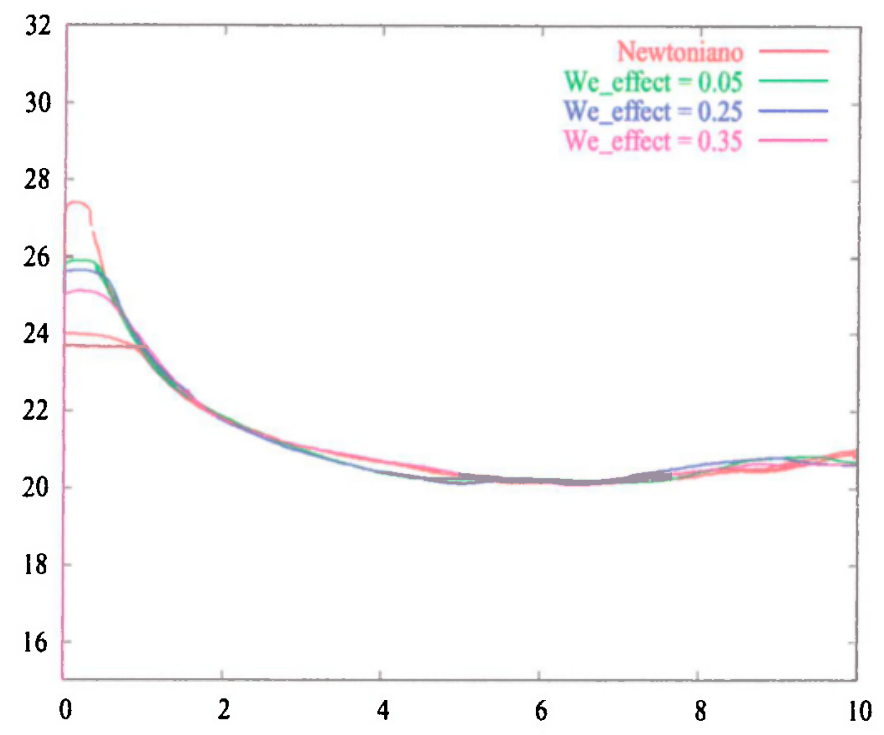

Figura 4.22: Altura máxima do 'Worthington jet' para diferentes valores de $W e_{\text {effect }}$. $R e=500$ e $W e=0.5$.

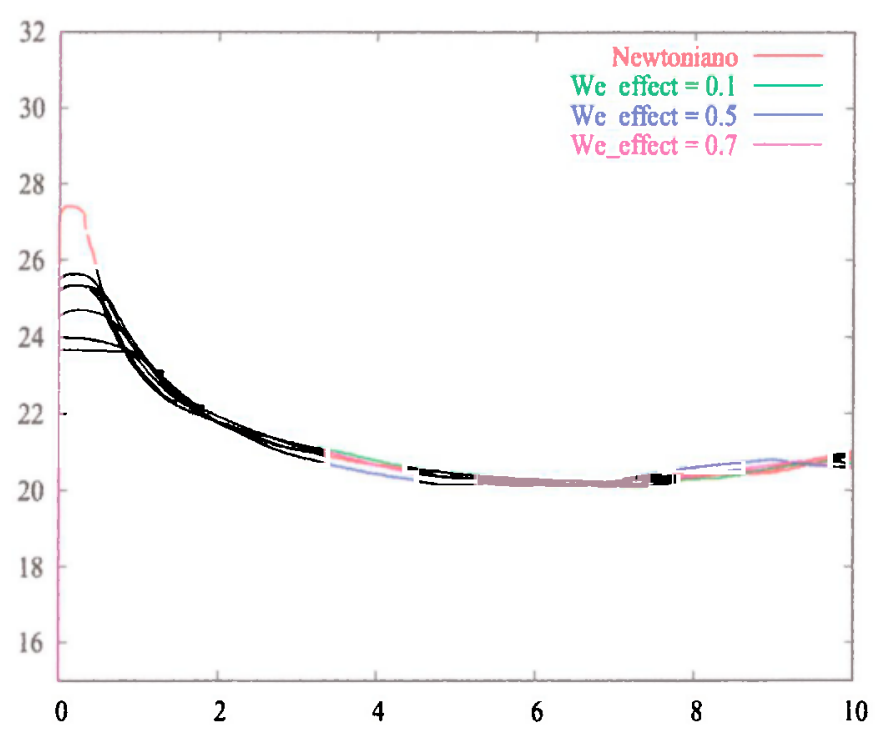

Figura 4.23: Altura máxima do 'Worthington jet' para diferentes valores de $W e_{\text {effect }}$. $R e=500$ e $W e=1.0$. 


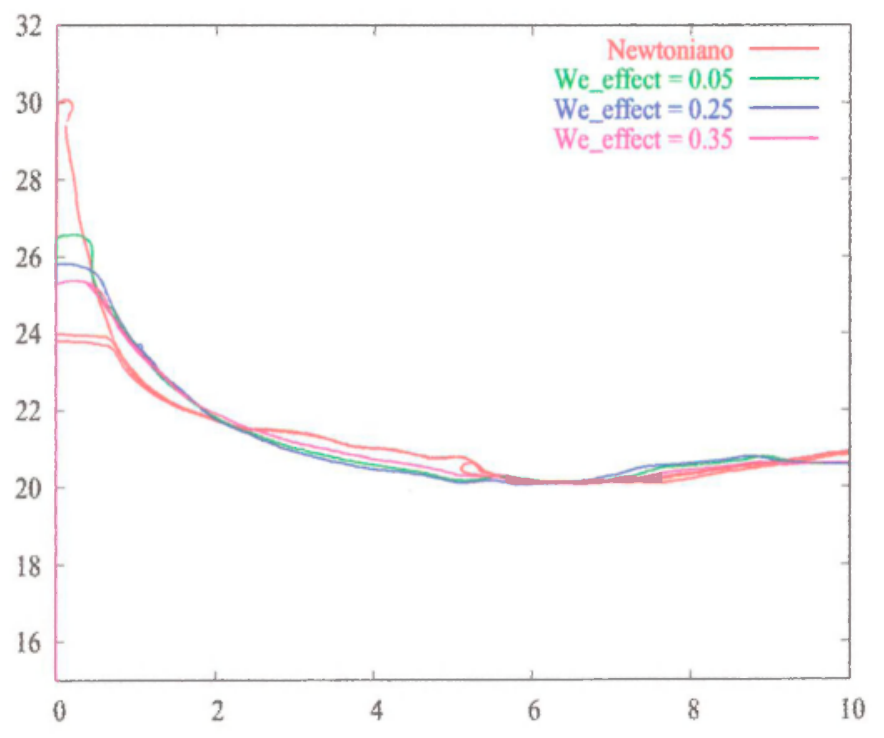

Figura 4.24: Altura máxima do 'Worthington jet' para diferentes valores de $W e_{\text {effect }}$ $R e=1000$ e $W e=0.5$.

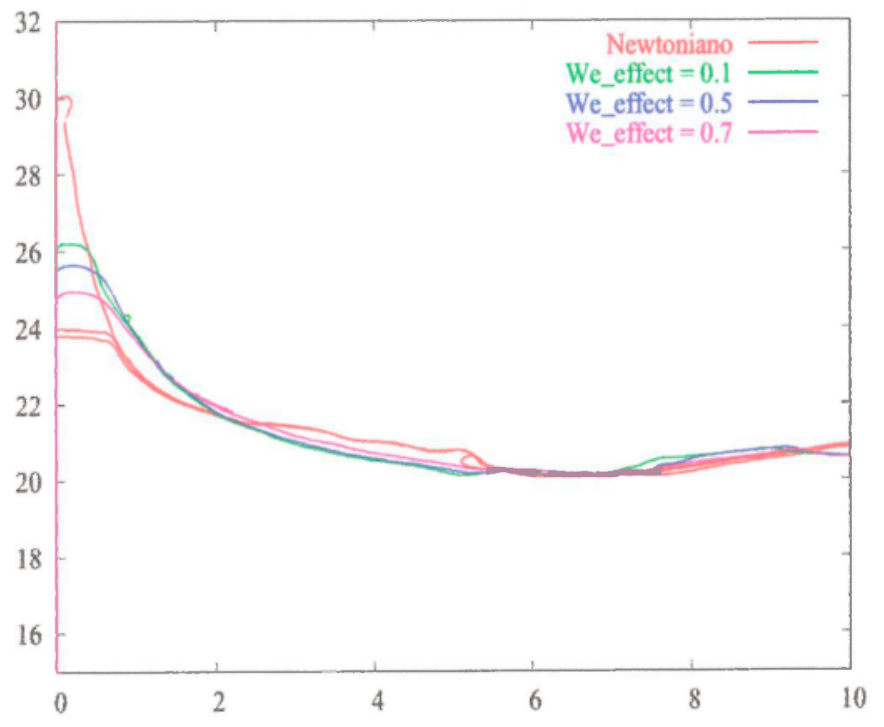

Figura 4.25: Altura máxima do 'Worthington jet' para diferentes valores de $W e_{\text {effect }}$. $R e=1000$ e $W e=1.0$. 


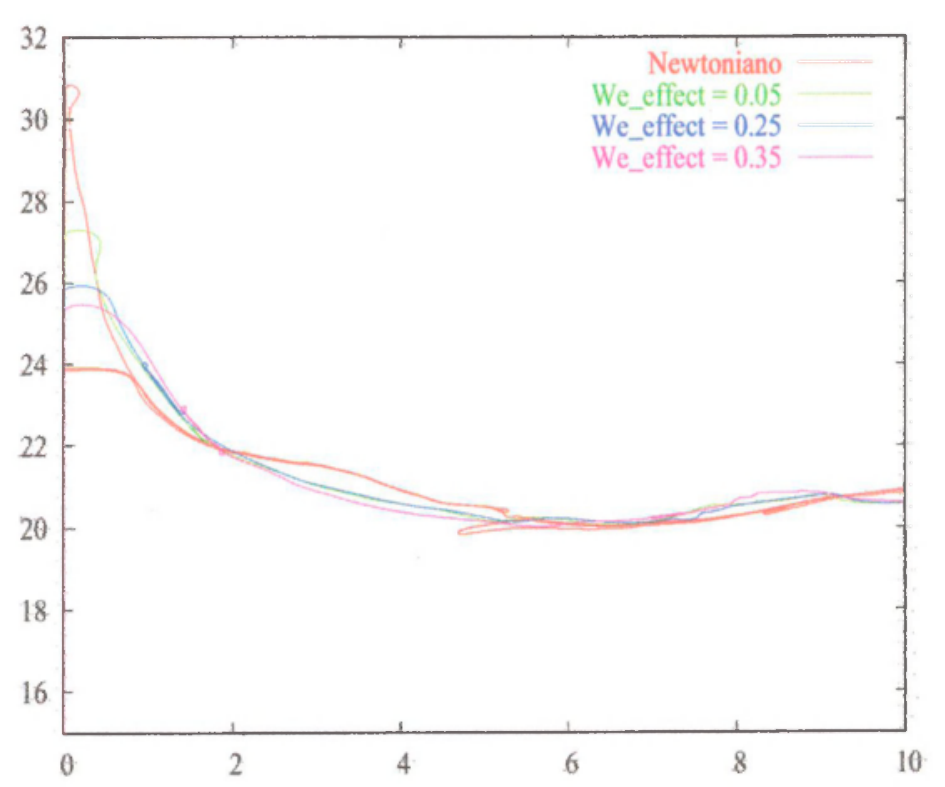

Figura 4.26: Altura máxima do 'Worthington jet' para diferentes valores de $W e_{\text {effect }}$. $R e=2000$ e $W e=0.5$.

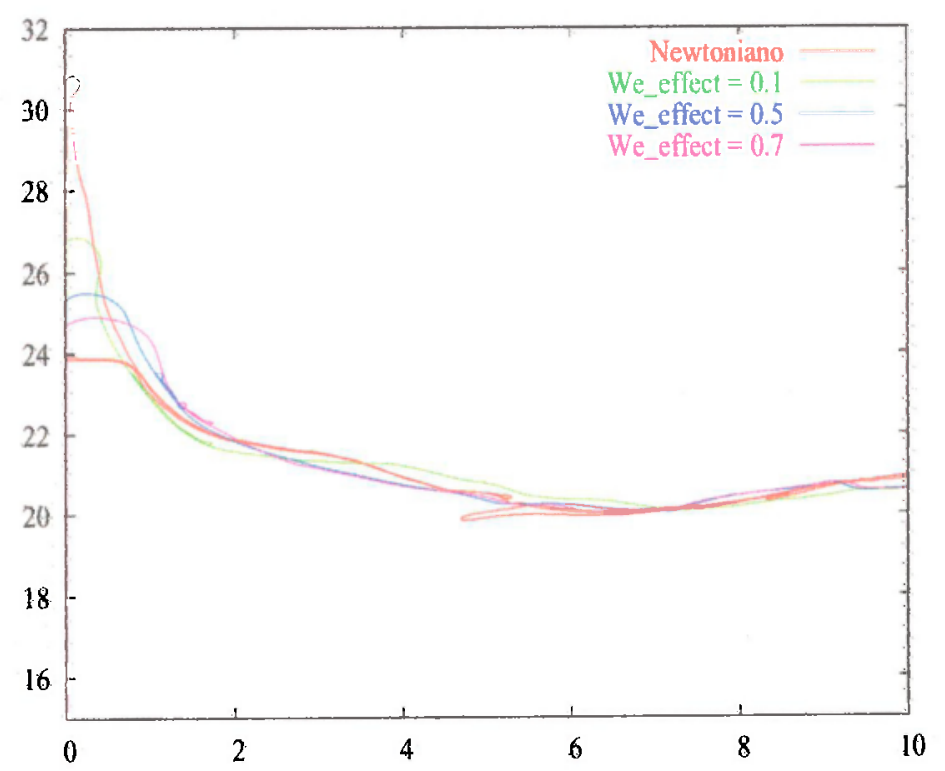

Figura 4.27: Altura máxima do 'Worthington' jet para diferentes valores de $W e_{\text {effect. }}$. $R e=2000$ e $W e=1.0$. 
Os resultados para $R e=500$ e $W e=0.5,1.0$, são mostrados nas figuras 4.22 e 4.23 onde é mostrada a configuração do jato no tempo em que o 'Worthington jet' atinge sua altura máxima. Podemos observar que a altura do 'Worthington jet' diminui quando aumentamos o valor de $W e_{\text {effect. }}$ É interessante notar ainda que para $W e_{\text {cffect }}$ pequenos (por ex. $W e_{\text {effect }}=0.05$ ) o decréscimo na altura do 'Worthington jet' é bem notável e a medida que aumentamos o valor de $W e_{\text {effect }}$ a altura do 'Worthington jet' produzido pelo modelo Oldroyd-B é reduzida e para $W e_{\text {effect }}=0.7$ a altura do 'Worthington jet' é menos que a metade da altura obtida com o fluido newtoniano. Estes resultados estão de acordo com os experimentos de Cheny \& Walters [26], [27].

Com o objetivo de confirmar esses resultados numéricos, um outro grupo de simulações do 'splashing drop' foi realizado utilizando mais dois valores de Reynolds, Re $=1000 \mathrm{e}$ $R e=2000$. Os valores de $W e$ e da razão $\frac{\lambda_{2}}{\lambda_{1}}$ escolhidos foram os mesmos que os utilizados no caso em que $R e=500$. Os resultados destas simulações são mostrados nas figuras 4.24 - 4.27. Por meio destas figuras podemos constatar que, como no caso em que $R e=500$, a altura do 'Worthington jet' diminui a medida que aumentamos o valor de $W e_{\text {effect }}$ e para o maior número de Reynolds utilizado, $R c=2000$, a altura do 'Worthington jet' foi maior que para os outros valores de $R e$. Estes resultados estão coerentes com os encontrados na literatura.

As figuras 4.28 - 4.33 mostram a visualização da altura máxima do 'Worthington jet' obtido para o caso newtoniano e para os casos viscoelásticos em que $R e=500,1000,2000$ e $W e=0.5,1.0$. 


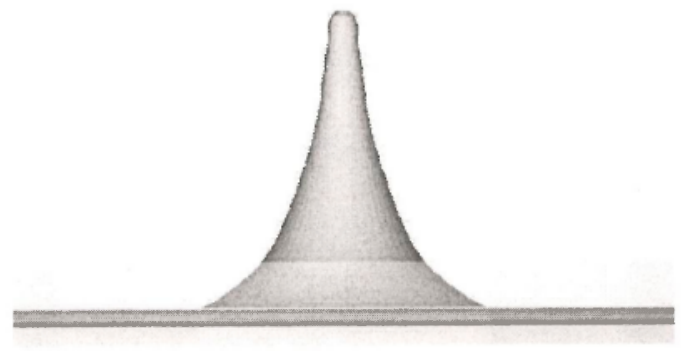

Newtoniano

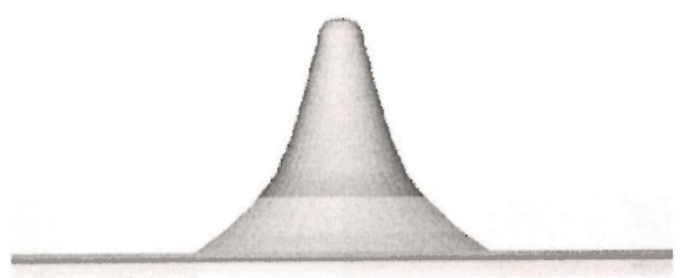

$W e_{\text {effect }}=0.05$

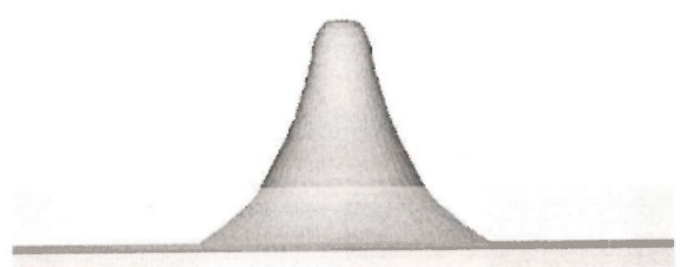

$W e_{\text {effect }}=0.25$

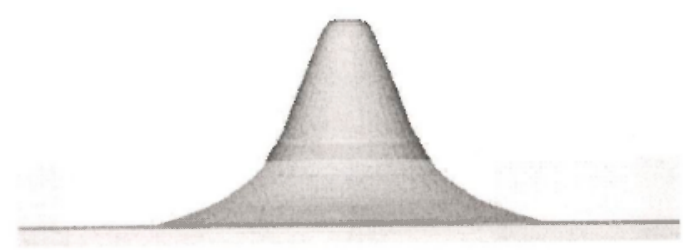

$W e_{\text {effect }}=0.35$

Figura 4.28: Visualização da simulação do 'splashing drop'. Altura máxima do 'Worthington jet' para $R e=500$ e $W e=0.5$. 


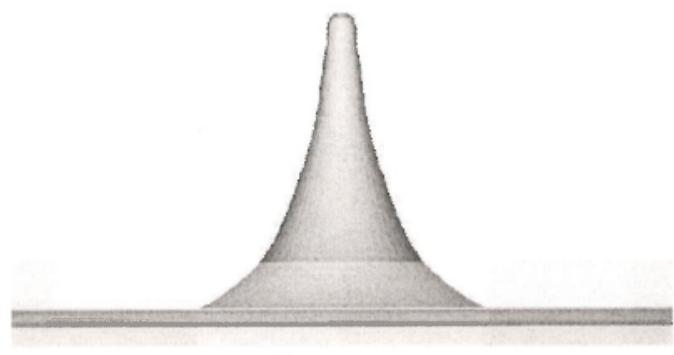

Newtoniano

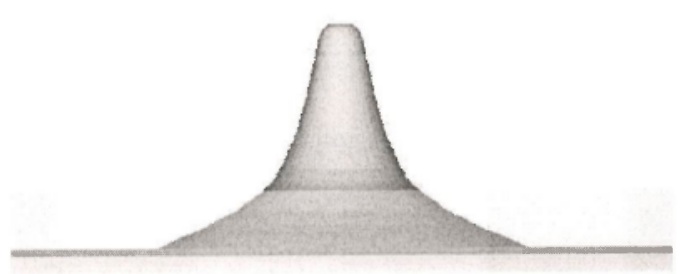

$W e_{\text {effect }}=0.1$

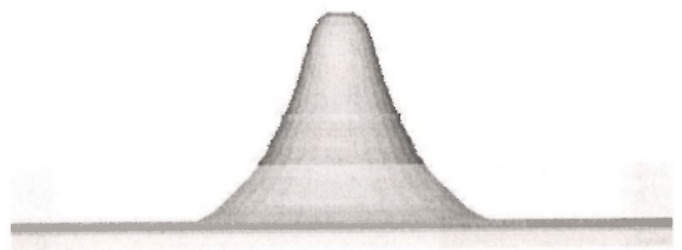

$W e_{\text {effect }}=0.5$

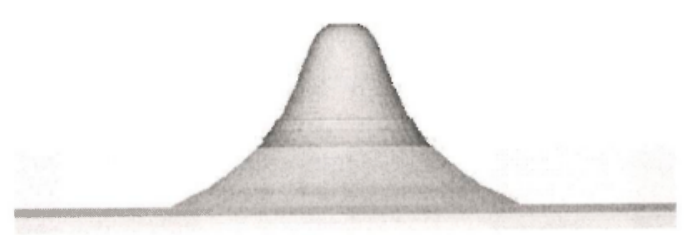

$W e_{\text {effect }}=0.7$

Figura 4.29: Visualização da simulação do 'splashing drop'. Altura máxima do 'Worthington jet' para $R e=500$ e $W e=1.0$. 


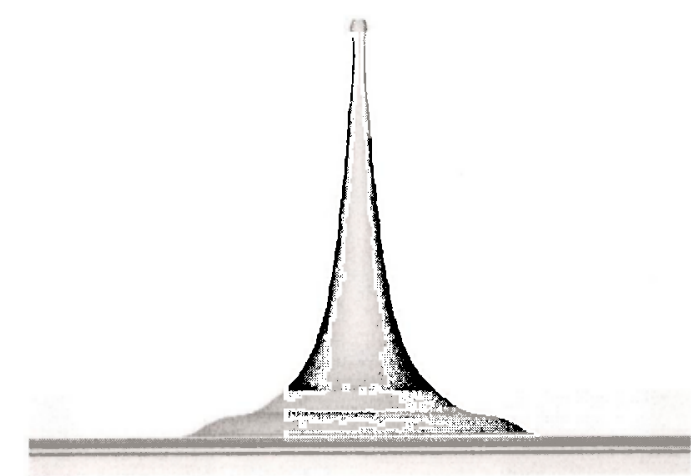

Newtoniano

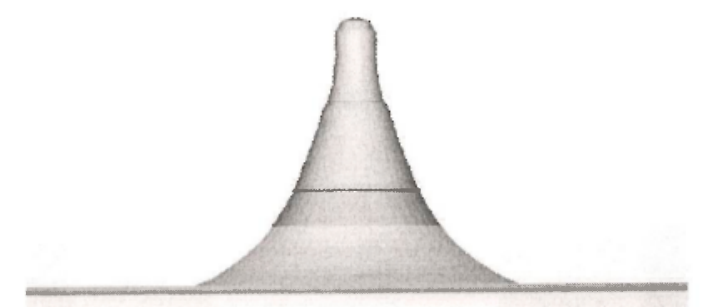

$W e_{\text {effect }}=0.05$

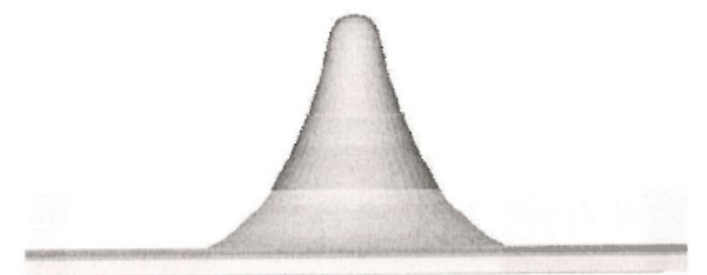

$W e_{\text {effect }}=0.25$

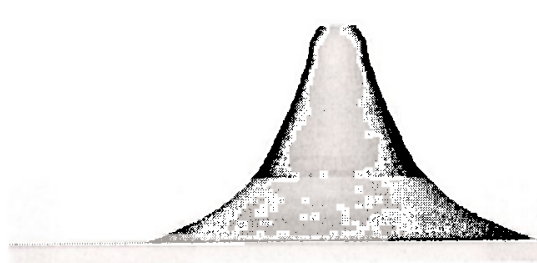

$W e_{\text {effect }}=0.35$

Figura 4.30: Visualização da simulação do 'splashing drop'. Altura máxima do 'Worthington jet' para $R e=1000$ e $W e=0.5$. 

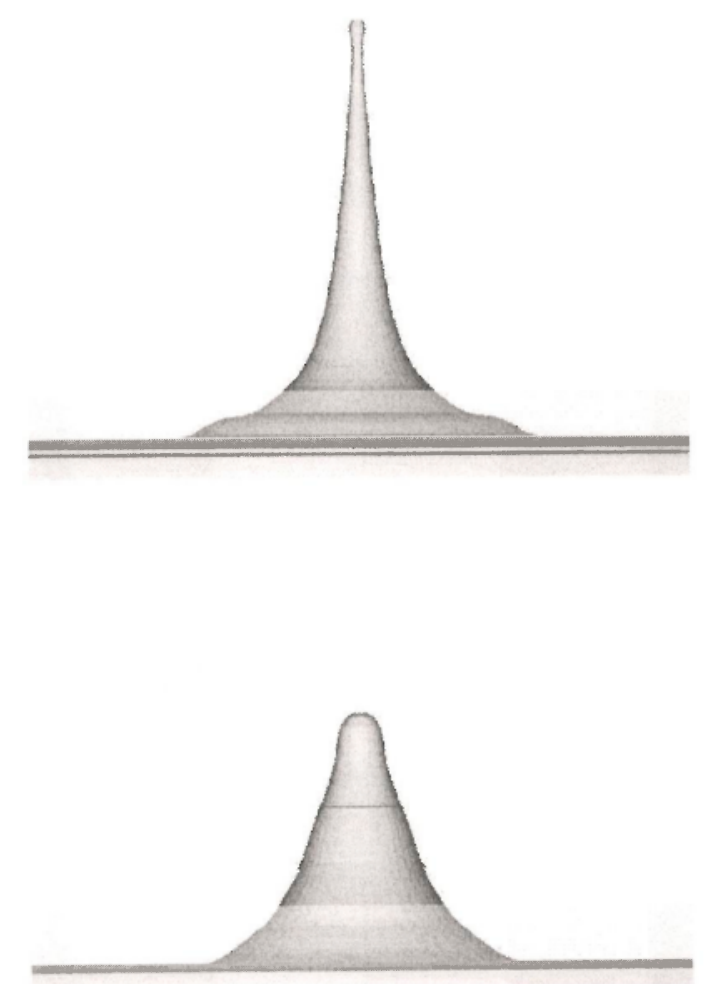

$W e_{\text {effect }}=0.1$

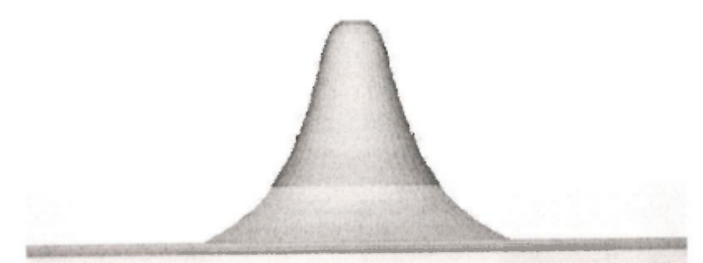

$W e_{\text {effect }}=0.5$

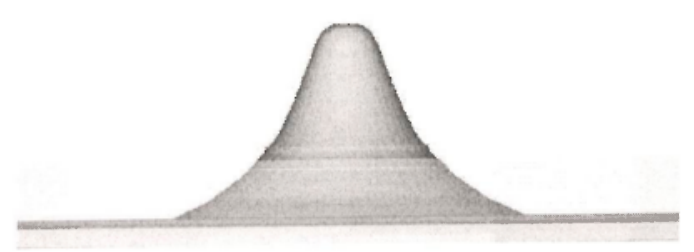

$W e_{\text {effect }}=0.7$

Figura 4.31: Visualização da simulação do 'splashing drop'. Altura máxima do 'Worthington jet' para $R e=1000$ e $W e=1.0$. 


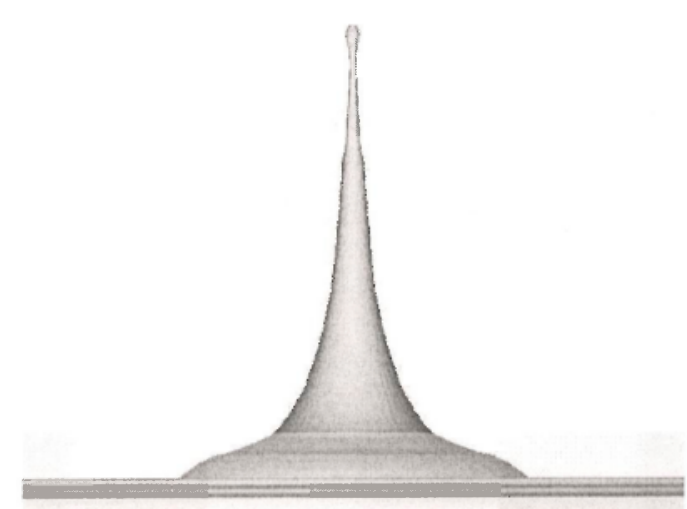

Newtoniano

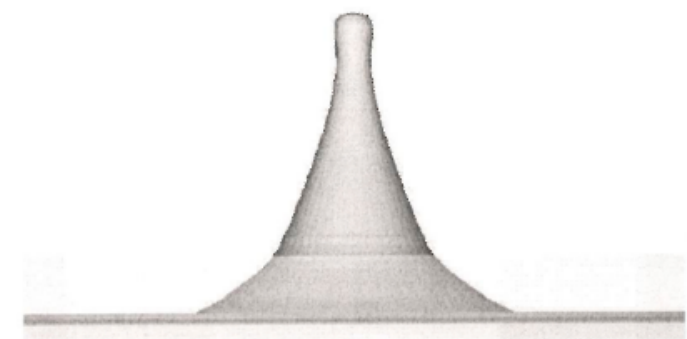

$W e_{\text {effect }}=0.05$

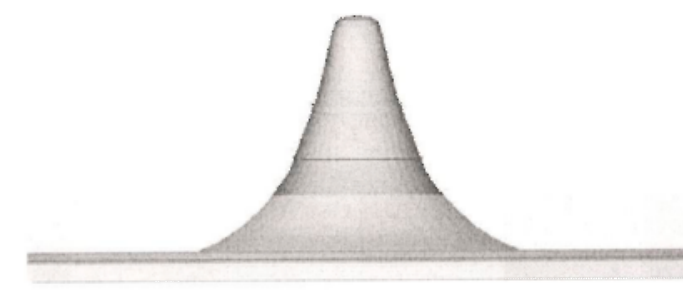

$W e_{\text {effect }}=0.25$

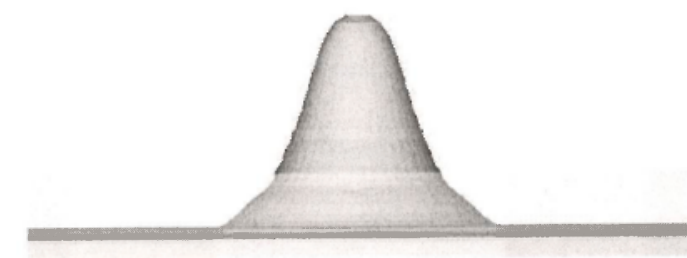

$W e_{\text {effect }}=0.35$

Figura 4.32: Visualização da simulação do 'splashing drop'. Altura máxima do 'Worthington jet' para $R e=2000$ e $W e=0.5$. 


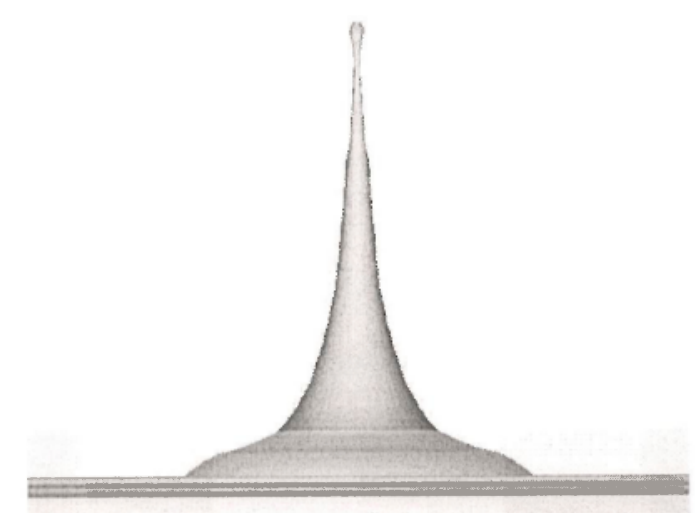

Newtoniano

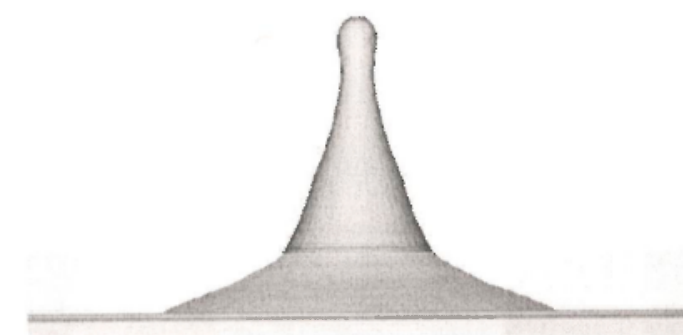

$W e_{\text {effect }}=0.1$

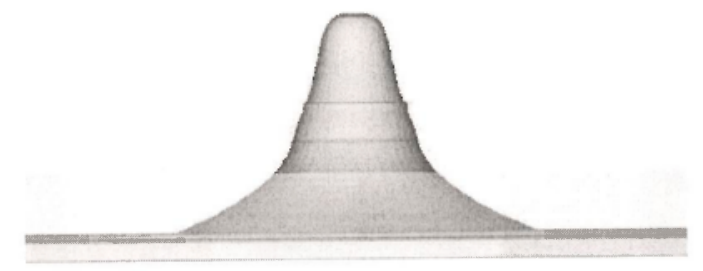

$W e_{\text {effect }}=0.5$

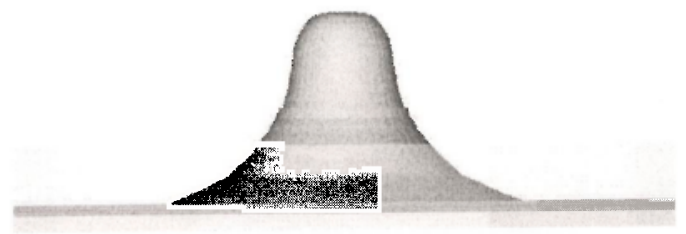

$W e_{\text {effect }}=0.7$

Figura 4.33: Visualização da simulação do 'splashing drop'. Altura máxima do 'Worthington jet' para $R e=2000$ e $W e=1.0$. 


\section{Conclusão}

O objetivo do trabalho apresentado nesta dissertação de mestrado consistiu em estender o ambiente de simulação Freeflow-AXI para simular escoamentos viscoelásticos com superfícies livres utilizando a equação constitutiva Oldroyd-B. Foi realizado um cstudo para o cálculo do tensor extra-tensão nas fronteiras rígidas e na fronteira que represcinta o eixo de simetria. Por meio deste tratamento foram obtidas expressões analíticas que representam as condições de contorno sobre estas fronteiras para cada componente do tensor extra-tensão. As equações provenientes do modelo Oldroyd-B foram resolvidas utilizando as idéias apresentadas por Tomé et al. [12] e L. Grossi [11].

A técnica de diferenças finitas desenvolvida neste trabalho foi validada utilizando o problema do escoamento totalmente desenvolvido em um tubo. Foram realizadas várias simulaçóes utilizando refinamento da malha e os resultados numéricos comparados com os respectivos resultados analíticos mostraram a convergência do método. Para mostrar que o inétodo numérico desenvolvido neste trabalho pode simular escoamentos viscoelásticos governados pelo modelo Oldroyd-B, foram simulados três problemas: inchamento do extrudado, impacto de uma gota incidindo contra uma superfície rígida e o 'splashing drop'. Os resultados obtidos na simulação do inchamento do extrudado estão coerentes com os encontrados na literatura. Os resultados da simulação de uma gota contendo fluido Oldroyd-B incidindo contra uma superfície rígida mostraram que ao atingir a superfície a gota começa a escoar radialmente e após algum tempo o escoamento muda de direção e a gota sofre uma contração. Acreditamos que cste efeito é devido aos efeitos viscoelásticos visto que, a simulação de uma gota contendo fluido newtoniano não apresentou este efeito. Por último, foram apresentados os resultados numéricos da simulação do 'splashing drop' de um fluido Oldroyd-B. Foram realizadas várias simulações variando o número de Reynolds e o número de Weissenberg efetivo. Os resultados mostraram que, a medida que aumentamos o número de Weissenberg efetivo (Weffect) a altura do 'Worthington jet' diminui quando comparada com a altura apresentada utilizando um fluido ncwtoniano. Novamente, acreditamos que este efeito ocorre devido aos efeitos viscoelásticos no escoamento. Esses resultados confirInam os dados experimentais apresentados por Chenny \& Walters [27]. 


\section{Referências Bibliográficas}

[1] M. F. Tomé, B. R. Duffy, S. Mckee, A numerical technique for solving unsteady Non-Newtonian free surface flows, J. Non-Neutonian Fluid Mech, 62:9-34; (1996).

[2] M. F. Tomé and S. McKee, GENSMAC: a computational marker-and-cell method for free surface flows in general domains, J. Comput. Phys., 110:171-186; (1994).

[3] M. F. 'omé, L. Grossi, A. Castelo, J. A. Cuminato, V. G. Ferreira, N. Mangiavacchi, $\mathrm{S}$. McKee, A numerical technique for simulating viscoelastic axisymmetric free surface flows, Proceedings of COBEMQ005, pág. 1-10-CDROM (2003).

[4] N. Mangiavacchi, A. Castelo, M. F. Tomé, J. A. Cuminato, M. L. B. de Oliveira, S. McKee, An effective implementation of surface tension using the marker and cell method for asisymmetric and planar flows, Siam J. on Scientific Computing, 26 (4):1340-1368; (2005).

[5] D. E. Morton, M. J. Rudman and J. L. Liow, A finite difference method for modelling impacting drops, FEDSM97, 3395, 1-6; (1997).

[6] V. Armenio, An improved MAC method (SIMAC) for unsteady high Reynolds free surface flows, Int. J. Numer. Meth. Fluids, 24:185-214; (1997).

[7] I. S. Kang and L. G. Leal, Numerical Sirnulation of Axisymmetric, Unsteady FreeBoundary Problem at Finite Reynolds Number. I. Finite-Difference Scheme and Its Aplication to the Deformation of o Buble in a Lniaxial Straining Flow, Phys. Fluids, 30:1929-1940; (1987).

|8] G. P. Sasmal, A finite volume approach for calculation of viscoclastic flow through an abrupt axisymmetric contraction, J. Non-Newtonian Fluid Mech, 56:15-47; (1995).

[9] A. Castelo, M. F. Tomé, C. N. L. César, S. McKee and J. A. Cuminato, Freeflow: An integrated simulation system for three-dimensional free surface flows, Computing and Visualization in Science, 2:199-210; (2000). 
[10] M. L. B. Oliveira, Freeflow-AXI: Um ambiente de simulação de escoamentos axissimétricos com superficies livres, São Carlos, ICMC-USP. Dissertação de Mestrado; (2002).

[11] L. Grossi, Desenvolvimento de métodos numéricos para a simulação de escoamentos não-Newtonianos e viscoelásticos com superfícies livres, São Carlos, ICMC-USP. Tese de Doulorado; (2003).

[12] M. F. Tomé, N. Mangiavacchi, A. Castelo, J. A. Cuminato and S. McKee, A finite difference technique for simulating unsteady viscoelastic free surface flows, J. NonNewtonian Fluid Mech, 106:61-106; (2002).

[13] M. Crochct and K. Walters, Numerical Simulation of Non-Newtonian Flow, Elsevier, New York, (1984).

[14] G. K. Batchelor, An Introduction to Fluid Dynamics, Cambridge University Press, Cambridge, (1967).

[15] G. Mompean and M. Deville, Unsteady finite volume of Oldroyd-B fluid through a three-dimensional planar contraction, J. Non-Newtonian Fluid Mech, 72:253-279; (1997).

[16] M. A. Alves, P. J. Oliveira, F. 'T. Pinho, A convergent and universally bounded interpolation scheme for the treatment of advection, Int. J. Numer. Meth. Fluids, 41:47-75; (2003).

[17] V. G. Ferreira, M. F. Tomé, N. Mangiavacchi, A. Castelo, J. A. Cuminato, A. O. Fortuna and S. McKee, High-order upwinding and the hydraulic jump, Int. J. Numer. Meth. Fluids, 39:549-583; (2002).

[18] T. N. Phillips and A. Williams, Viscoelastic flow though a planar contraction using a semi-Lagrangian finite volume method, J. Non-Newtonian Fluid Mech., 87:215-246; (1999)

[19] J. Yoo and Y. Na, A numerical study of the planar contraction flow of a viscoelastic fluid using the SIMPLER algorithm, J. Non-Newtonian Fluid Mech., 30:89-106, (1991).

[20] R. I. Tanner, A theory of die-swell, J. Polymer Science, 8:2067-2078; (1970).

[21] M. E. Ryan and A. Dutra, A finite Difference simulation of Extrudate Swell, Proc. 2nd. World Congr.Chem. Eng., VI:277-281; (1981).

[22] M. J. Crochet and R. Keunings, Finite element analysis of dieswell of a highly clastic fluid, J. Non-Newtonian Fluid Mech., 10:339-356; (1982). 
[23] M. A. Worthington and R. S. Cole, Impact with a liquid surface studied by the aid of instantaneous photography, Phil. Trans. R. Soc. A189 (1897), 137-148.

[24] M. A. Worthington and R. S. Cole, Impact with a liquid surface studied by the aid of instantaneous photography, Paper II, Phil. Trans. R. Soc. A194, 175-199; (1900).

[25] M. A. Worthington, A study of Splashes, Longmansand Gren, London, 1908.

[26] J. M. Cheny and K. Walters, Extravagant viscoelastic effects in the Worthington jet experiment, J. Non-Newtonian Fluid Mech., 67:125-135; (1996).

[27] J. M. Cheny and K. Walters, Rheological influences on the splashing experiment, $J$. Non-Newtonian Fluid Mech., 86:185-210; (1999).

[28] S. Nigen and K. Walters, On the two-dimensional splashing experiment for Newtonian and slightly elastic liquids, J. Non-Newtonian Fluid Mech., 97:233-250; (2001).

[29] M. Mäntylä, An introduction to Solid Modeling, Computer Science Press, 1988. 